\title{
Dynamic aggregation operators and Einstein operations based on interval-valued picture hesitant fuzzy information and their applications in multi-period decision making
}

\author{
Hüseyin Kamacı ${ }^{1}$ Subramanian Petchimuthu ${ }^{2}$ Eyüp Akçetin ${ }^{3}$
}

Received: 18 November 2020 / Revised: 2 April 2021 / Accepted: 10 April 2021 /

Published online: 29 April 2021

(c) SBMAC - Sociedade Brasileira de Matemática Aplicada e Computacional 2021

\begin{abstract}
The traditional picture hesitant fuzzy aggregation operators are generally suitable for aggregating information acquired in the form of picture hesitant fuzzy numbers, but they will fail in dealing with interval-valued picture hesitant fuzzy information. In this paper, we describe the notion of interval-valued picture hesitant fuzzy set and the operational laws of intervalvalued picture hesitant fuzzy variables. Moreover, we derive some dynamic interval-valued picture hesitant fuzzy aggregation operators (based on Einstein operators) to aggregate the interval-valued picture hesitant fuzzy information collected at different periods. Some desirable properties of these aggregation operators are discussed in detail. In addition, we develop the approaches to tackle the multi-period decision-making problems, where all decision information takes the form of interval-valued picture hesitant fuzzy information collected at different periods. In an attempt to illustrate the applications of the proposed approaches, two numerical examples are given to measure the impact of Coronavirus Disease 2019 (COVID19 ) in daily life and to identify the optimal investment opportunity. Finally, a comparative analysis of the proposed and existing studies are conducted to demonstrate the effectiveness of the proposed approaches. The presented interval-valued picture hesitant fuzzy operations, aggregation operators, and decision-making approaches can widely apply to dynamic decision analysis and multi-stage decision analysis in real life.
\end{abstract}

Keywords Interval-valued picture hesitant fuzzy set · Dynamic aggregation operator · Einstein operation $\cdot$ COVID-19 $\cdot$ Multi-period decision making

Mathematics Subject Classification 03E72 · 94D05 · 91B06

Communicated by Marcos Eduardo Valle.

$\bowtie \quad$ Hüseyin Kamacı

huseyin.kamaci@hotmail.com

1 Department of Mathematics, Yozgat Bozok University, 66100 Yozgat, Turkey

2 Department of Science and Humanities (Mathematics), University College of Engineering, Nagercoil, Tamilnadu 629004, India

3 Department of Accounting and Financial Management, Seydikemer School of Applied Sciences, Mug̃la Sıtkı Koçman University, Mug̃la, Turkey 


\section{Introduction}

In 1965, Zadeh (1965) introduced the idea of fuzzy logic which is the expansion of binary logic. When handling a few involute systems, due to the flexibility of membership of fuzzy logic, many generalized styles of the fuzzy set (FS) and fuzzy element (FE) were developed in the following year. Some of these are as follows: interval-valued fuzzy set (IVFS) Sambuc (1975), in which value of membership in the fuzzy set is interval; intuitionistic fuzzy set (IFS) Atanassov (1986, 1989), which is created by integrating the function of non-membership into the form of a fuzzy set; interval-valued intuitionistic fuzzy set (IVIFS) Atanassov and Gargov (1989), in which values of membership and non-membership in the intuitionistic fuzzy set are intervals. These paradigms were studied by many researchers in various aspects (Kamac1 2019; Karaaslan and Karataş 2016; Khan and Zhu 2020; Khan et al. 2019a; Petchimuthu et al. 2020; Petchimuthu and Kamac1 2020; Riaz and Tehrim 2019, 2020). Many researchers dealt with the extended types of IFSs that stretch the underlying requirement of IFS that the sum of membership degree and non-membership degree is less than or equal to 1 (Naeem et al. 2019; Riaz et al. 2020; Wang et al. 2020; Wang and Li 2020). In 2010, Torra (2010) introduced the notion of a hesitant fuzzy set, which permits the membership to have a set of possible values. This paradigm allows overcoming some situations in which experts hesitate between several possible membership values to assess an alternative. Xia and Xu (2011) defined a few operational regulations for HFS based on the algebraic t-norm and t-conorm, and then advanced several hesitant fuzzy aggregation operators to aggregate hesitant fuzzy information. Many authors (Deli and Karaaslan 2020; Wei 2012; Zhang 2013a; Zhu et al. 2012b) developed different types of aggregation operators to aggregate the possible values of membership of alternatives in the decision making under the surrounding of HFS. Chen et al. (2013) and Wei et al. (2013) generalized the framework of the HFS to the interval-valued hesitant fuzzy set (IVHFS). They argued that the membership degrees in an IVHFS can be both the set of exact fuzzy numbers and the set of interval-valued fuzzy numbers. Jin et al. (2016) advanced the Einstein prioritized aggregation operators for the interval-valued hesitant fuzzy variables. Beg and Rashid (2014) blended the notions of IFS and HFS to create a brand new perception referred to as the intuitionistic hesitant fuzzy set (IHFS). Zhang (2013b) studied the intervalvalued intuitionistic hesitant fuzzy set (IVIHFS), which is the generalized form of IHFSs, and their basic properties. Zhu et al. (2012a) proposed the idea of hybridization of a hesitant fuzzy set with duality, and thus initiated a dual hesitant fuzzy set (DHFS) including possible values of membership and non-membership. They investigated some fundamental operations and properties of DHFS. Moreover, Wang et al. (2014) advanced a few aggregation operators based on dual hesitant fuzzy elements (DHFEs), such as the dual hesitant fuzzy weighted average, weighted geometric, ordered weighted average, ordered weighted geometric, hybrid average, and hybrid geometric operators. In Ju et al. (2014b), the authors derived some dual hesitant fuzzy Hamacher average and geometric aggregation operators to apply in a multiple attribute decision-making problem. Ju et al. (2014a) generalized the dual hesitant fuzzy set to interval-valued dual hesitant fuzzy set (IVDHFS) and developed the interval-valued dual hesitant fuzzy aggregation operators. Moreover, Zhang et al. (2014) published a paper on the interval-valued dual hesitant fuzzy Einstein weighted aggregation operators like ordered weighted average operator, ordered weighted geometric operator, hybrid average operator, and hybrid geometric operators. On the other hand, Cuong (2014) proposed integrating the grade of indeterminacy into the structure of IFS and defined a picture fuzzy set (PFS), which is characterized by the degrees of positive membership, neutral membership (or indeterminacy), and negative membership (or non-membership). They pointed out that the simplest 
constraint is that the sum of the three degrees must not exceed 1. This constraint allows PFSs to have a different structure than neutrosophic sets (see: Akram et al. 2018; Kamac1 2020; Riaz and Hashmi 2020; Smarandache 1998; Tehrim and Riaz 2019 for neutrosophic sets). In addition, many researchers derived various aggregation operators to aggregate the picture fuzzy aggregation information, e.g., the picture fuzzy aggregation operators and picture fuzzy Hamacher aggregation operators (Wei 2017, 2018), the picture fuzzy aggregation operators based on Einstein operations (Khan et al. 2019b), and the picture fuzzy hybrid averaging operator (Garg 2017). In Khalil et al. (2019), a generalization of the concept of picture fuzzy set was given in the spirit of ordinary interval-valued fuzzy sets, and thus initiated the theory of interval-valued picture fuzzy set (IVPFS). Wang and Li (2018) introduced picture hesitant fuzzy set (PHFS) by combining the PFS and HFS, and proposed the generalized picture hesitant fuzzy weighted aggregation operators and the generalized picture hesitant fuzzy prioritized weighted aggregation operators to solve the multi-criteria decision-making (MCDM) problems.

In general, the above studies were dedicated to proposing models aggregating the hesitant/picture/picture hesitant fuzzy information in the same period. However, some complex decision-making problems have to consider the performance of alternatives at the different periods. Such problems are said to be multi-period decision-making (MPDM) problems, where information is collected in time moments of a period. In recent years, many authors investigated the temporal generalized versions (often called dynamic) of the existing hesitant/picture/picture hesitant fuzzy aggregation operators and discussed their efficiency in the MPDM. For instance, Lin et al. (2008) created a dynamic multi-attribute decision-making model with grey number evaluations, Peng and Wang (2014) proposed dynamic hesitant fuzzy aggregation operators to deal with multi-period decision-making problems. While the current picture hesitant fuzzy aggregation operators are suitable for aggregating the picture hesitant fuzzy information collected at the same period, these aggregation operators cannot be used to deal with MPDM problems under the picture hesitant fuzzy environment.

Based on the above analysis, we find that how to extend the PHFS to interval-valued picture hesitant fuzzy set (IVPHFS), where the degrees of positive membership, neutral membership, and negative membership are the sets of possible fuzzy intervals and how to aggregate the interval-valued picture hesitant fuzzy information collected at different periods are meaningful research issues. This paper is motivated to fill these research gaps in the literature. Now, we discuss some important objectives of this study.

1. In some real-life problems based on the nature of PHFS, the degrees of positive membership, neutral membership, and negative membership may be the sets of possible fuzzy intervals. The PHFS fail in such situations. To overcome these deficiencies, the concept of IVPHFS that extends the intuitive, hesitant, and picture hybridizations of fuzzy sets is defined. The relationship among interval-valued picture hesitant fuzzy set and several existing fuzzy sets is presented in Fig. 1.

The IVPHFSs allow the membership of objects that cannot be characterized by the existing fuzzy sets (in Fig. 1) to be clearly described. In addition, the basic operations and relations presented for IVPHFSs and IVPHFEs contribute to both theory and practice in fuzzy mathematics.

2. The existing types of picture hesitant fuzzy aggregation operators are suitable for aggregating the picture hesitant fuzzy information collected in the same period. But these existing aggregation operators cannot deal with situations, where information is collected at the different periods. To fuse interval-valued picture hesitant fuzzy information collected at the different periods, the dynamic interval-valued picture hesitant fuzzy aggregation oper- 


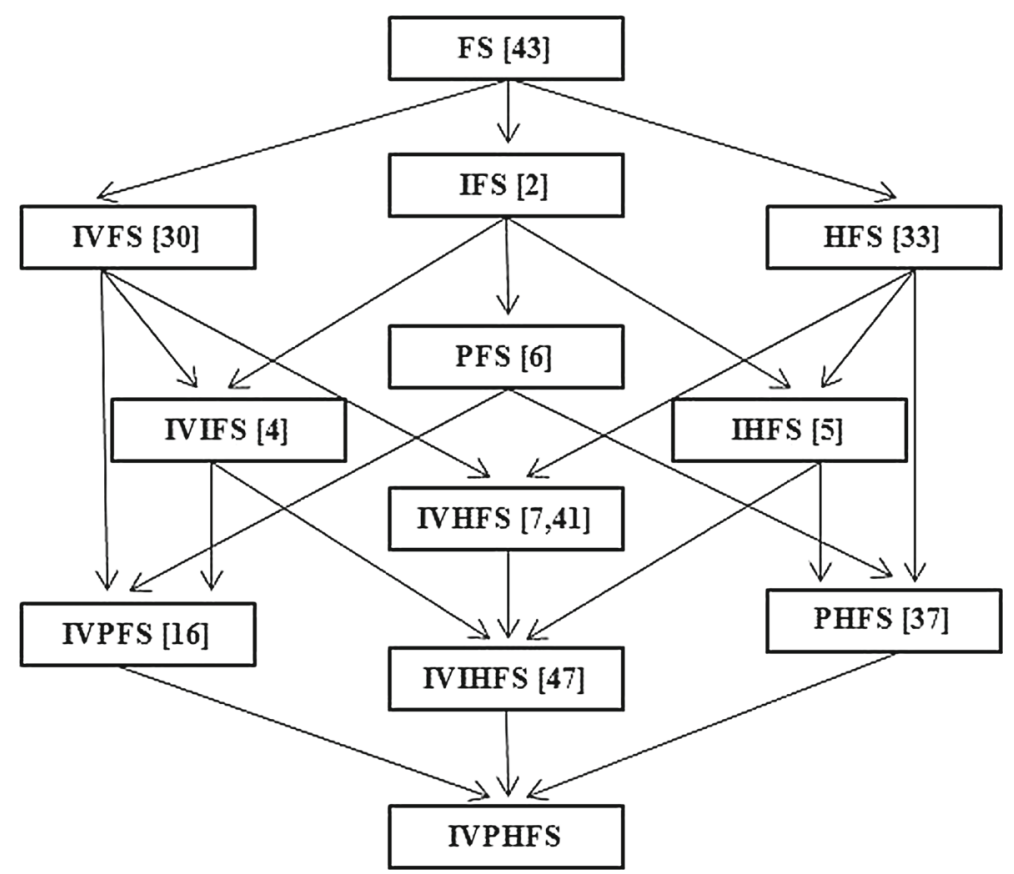

Fig. 1 Relationship among interval-valued picture hesitant fuzzy set and other fuzzy sets

ators (based on Einstein operations) and their generalized forms are proposed. Each of these proposed dynamic aggregation operators proceeds differently during calculations, and so it is put forward that in some cases one can be superior to the other. It is obvious that if it is taken as $t=t_{1}$ (i.e., the period has only one time moment) in the formulations of dynamic interval-valued picture hesitant fuzzy aggregation operators then these dynamic aggregation operators turn into traditional interval-valued picture hesitant fuzzy aggregation operators. Thus, it is highlighted that the developed dynamic aggregation operators aggregate interval-valued picture hesitant fuzzy information collected both at the same period and the different periods.

3. It is not possible to solve the multi-period decision-making problems using the existing picture hesitant fuzzy arguments. This study proposes the approaches using the developed dynamic interval-valued picture hesitant fuzzy aggregation operators to deal with diverse situations during multi-period decision-making process under the interval-valued picture hesitant fuzzy environment. In addition, it is shown that these approaches can be applied to real-life problems such as measuring the impact of COVID-19 and determining the optimal choice of investment.

The remainder of the paper is prepared as follows: in Sect. 2, the concept of IVPHFS and some interval-valued picture hesitant fuzzy aggregation operators that can be used to fuse time-independent interval-valued picture hesitant fuzzy arguments are introduced. Section 3 describes some new dynamic interval-valued picture hesitant fuzzy weighted aggregation operators and their generalized versions to aggregate interval-valued picture hesitant fuzzy information collected at different periods. In Sect. 4, the notions of (generalized) dynamic interval-valued picture hesitant fuzzy weighted aggregation operators based on Einstein operations and their essential properties such as idempotency, boundedness, and monotonicity 
are given. Section 5 proposes two approaches using new dynamic aggregation operators to the MPDM, where all decision information takes the form of interval-valued picture hesitant fuzzy information collected at different periods. The numerical examples are given to illustrate how to apply the developed decision-making approaches. Section 6 is devoted to the comparative analysis, followed by concluding remarks in Sect. 7.

\section{Interval-valued picture hesitant fuzzy set}

In this part, we introduce the concepts of interval-valued picture hesitant fuzzy set and interval-valued picture hesitant fuzzy element. Moreover, we give some essential notions (like union, intersection, aggregation operators) for interval-valued picture hesitant fuzzy elements.

From now on, $I^{[0,1]}$ denotes the set of all closed subintervals of $[0,1]$. If $x \in I^{[0,1]}$ then $x=\left[x^{L}, x^{U}\right]$, such that $x^{L} \leq x^{U}$.

Definition 2.1 Let $Q$ be a nonempty and finite set. $I^{[0,1]}$ denotes the set of all closed subintervals of $[0,1]$. Then, an interval-valued picture hesitant fuzzy set (IVPHFS) $\widetilde{P}$ in $Q$ is defined as

$$
\widetilde{P}=\{\langle q, \widetilde{\wp}(q)\rangle: q \in Q\}=\{\langle q, \widetilde{\alpha}(q), \widetilde{\beta}(q), \widetilde{\gamma}(q)\rangle: q \in Q\}
$$

where $\widetilde{\alpha}, \widetilde{\beta}, \widetilde{\gamma}$ are, respectively, the positive membership function, neutral membership function, and negative membership function of the set $\widetilde{P}$, and each of them when applied to $Q$ returns a subset of $I^{[0,1]}$. For each $q \in Q, \widetilde{\alpha}(q), \widetilde{\beta}(q)$ and $\widetilde{\gamma}(q)$ are the sets of some closed subintervals of $[0,1]$, denoting the possible degrees of positive membership, neutral membership, and negative membership of $q \in Q$ to the set $\widetilde{P}$, respectively. That is $\widetilde{\alpha}(q)=\left\{a: a=\left[a^{L}, a^{U}\right] \in I^{[0,1]}\right\}, \widetilde{\beta}(q)=\left\{b: b=\left[b^{L}, b^{U}\right] \in I^{[0,1]}\right\}$ and $\tilde{\gamma}(q)=\left\{c: c=\left[c^{L}, c^{U}\right] \in I^{[0,1]}\right\}$ with the following condition

$$
0 \leq a_{\max }^{U}+b_{\max }^{U}+c_{\max }^{U} \leq 1
$$

where

$a_{\max }^{U}=\bigcup_{\left[a^{L}, a^{U}\right] \in \tilde{\alpha}(q)} \max \left\{a^{U}\right\}, b_{\max }^{U}=\bigcup_{\left[b^{L}, b^{U}\right] \in \tilde{\beta}(q)} \max \left\{b^{U}\right\} a n d c_{\max }^{U}=\bigcup_{\left[c^{L}, c^{U}\right] \in \tilde{\gamma}(q)} \max \left\{c^{U}\right\}$.

For convenience, the triplet $\widetilde{\wp}(q)=\{\widetilde{\alpha}(q), \widetilde{\beta}(q), \widehat{\gamma}(q)\}$ is called an interval-valued picture hesitant fuzzy element (IVPHFE), denoted by

$$
\widehat{\wp}=\{\widehat{\alpha}, \widehat{\beta}, \widehat{\gamma}\}=\underset{\left[a^{L}, a^{U}\right] \in \widehat{\alpha},\left[b^{L}, b^{U}\right] \in \widehat{\beta},\left[c^{L}, c^{U}\right] \in \widehat{\gamma}}{\bigcup}\left\{\left\{\left[a^{L}, a^{U}\right]\right\},\left\{\left[b^{L}, b^{U}\right]\right\},\left\{\left[c^{L}, c^{U}\right]\right\}\right\}
$$

or

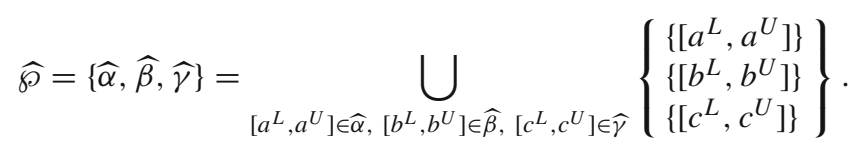

Throughout this paper, the term IVPHFE may sometimes appear as interval-valued picture hesitant fuzzy variable (IVPHFV) or interval-valued picture hesitant fuzzy number (IVPHFN). $\mathcal{P}$ denotes the set of all IVPHFEs (or IVPHFVs/IVPHFNs) on $Q$. 
Example 2.2 Air pollution is due to many reasons such as industrial wastes and fumes, heat and fume of vehicles and machinery, uncontrolled thermal power plants, and irregular heating systems (excessive coal consumption, and so on). Considering the areal and temporal variability of air pollution and the uncertainties that cause pollution, it may not be sufficient to create the membership (or non-membership) degree based on the fuzzy logic or the intuitionistic fuzzy logic for the air pollution of each city. This can be considered as an illustrative example of interval-valued picture hesitant fuzzy logic.

Let $Q=\left\{q_{1}=\right.$ Ankara, $q_{2}=$ Istanbul, $q_{3}=$ Yozgat, $q_{4}=$ Antalya $\}$ be a set of five cities in Turkey. According to "air pollution" of the cities, we can create the IVPHFS as follows:

$$
\widetilde{P}=\left\{\begin{array}{l}
\left\langle q_{1},\{[0.2,0.4]\},\{[0.1,0.3],[0.2,0.4]\},\{[0,0.1],[0.1,0.2]\}\right\rangle, \\
\left.\left\langle q_{2},\{[0.1,0.3]\},\{[0.2,0.2],[0.3,0.4],[0.2,0.5]\},\{[0,0.1]\}\right\}\right\rangle, \\
\left\langle q_{3},\{[0.2,0.35],[0.3,0.4]\},\{[0.1,0.25],[0.2,0.3]\},\{[0.1,0.2]\}\right\rangle, \\
\left\langle q_{4},\{[0.1,0.3],[0.2,0.5]\},\{[0.2,0.2]\},\{[0.1,0.1]\}\right\rangle
\end{array}\right\}
$$

Definition 2.3 Let $\widehat{\wp}=\{\widehat{\alpha}, \widehat{\beta}, \widehat{\gamma}\}=\bigcup_{\left[a^{L}, a^{U}\right] \in \widehat{\alpha},\left[b^{L}, b^{U}\right] \in \widehat{\beta},\left[c^{L}, c^{U}\right] \in \widehat{\gamma}}\left\{\left\{\left[a^{L}, a^{U}\right]\right\},\left\{\left[b^{L}, b^{U}\right]\right\}\right.$, $\left.\left\{\left[c^{L}, c^{U}\right]\right\}\right\}$ be an IVPHFE, the numbers of values in $\widehat{\alpha}, \widehat{\beta}, \widehat{\gamma}$ are $v(\widehat{\alpha}), v(\widehat{\beta}), v(\widehat{\gamma})$, respectively.

(i) The score function is defined as

$$
\begin{aligned}
\mathcal{S}(\widehat{\wp}) & =\frac{1+\frac{1}{2}\left(\frac{1}{v(\widehat{\alpha})} \sum_{\left[a^{L}, a^{U}\right] \in \widehat{\alpha}}\left(a^{L}+a^{U}\right)-\frac{1}{v(\widehat{\beta})} \sum_{\left[b^{L}, b^{U}\right] \in \widehat{\beta}}\left(b^{L}+b^{U}\right)-\frac{1}{v(\widehat{\gamma})} \sum_{\left[c^{L}, c^{U}\right] \in \widehat{\gamma}}\left(c^{L}+c^{U}\right)\right)}{2}, \\
\mathcal{S}(\widehat{\wp}) \in[0,1] &
\end{aligned}
$$

(ii) The accuracy function is defined as

$$
\begin{aligned}
& \mathcal{A}(\widehat{\wp})=\frac{1}{2}\left(\frac{1}{v(\widehat{\alpha})} \sum_{\left[a^{L}, a^{U}\right] \in \widehat{\alpha}}\left(a^{L}+a^{U}\right)+\frac{1}{v(\widehat{\beta})} \sum_{\left[b^{L}, b^{U}\right] \in \widehat{\beta}}\left(b^{L}+b^{U}\right)+\frac{1}{v(\widehat{\gamma})} \sum_{\left[c^{L}, c^{U}\right] \in \widehat{\gamma}}\left(c^{L}+c^{U}\right)\right), \\
& \mathcal{A}(\widehat{\wp}) \in[0,1]
\end{aligned}
$$

(iii) The certainty function is defined as

$$
\mathcal{C}(\widehat{\wp})=\frac{1+\frac{1}{2}\left(\frac{1}{v(\widehat{\alpha})} \sum_{\left[a^{L}, a^{U}\right] \in \widehat{\alpha}}\left(a^{L}+a^{U}\right)-\frac{1}{v(\widehat{\gamma})} \sum_{\left[c^{L}, c^{U}\right] \in \widehat{\gamma}}\left(c^{L}+c^{U}\right)\right)}{2}, \mathcal{C}(\widehat{\wp}) \in[0,1]
$$

Using the notions of score function, accuracy function and certainty function of IVPHFE, the two IVPHFEs can be compared as follows:

Definition 2.4 Let $\widehat{\wp}_{1}=\left\{\widehat{\alpha}_{1}, \widehat{\beta}_{1}, \widehat{\gamma}_{1}\right\}$ and $\widehat{\wp}_{2}=\left\{\widehat{\alpha}_{2}, \widehat{\beta}_{2}, \widehat{\gamma}_{2}\right\}$ be two IVPHFEs, then one can compare them in terms of the following rules:

1. if $\mathcal{S}\left(\widehat{\wp}_{1}\right)<\mathcal{S}\left(\widehat{\wp}_{2}\right)$ then $\widehat{\wp}_{1} \prec \widehat{\wp}_{2}$,

2. if $\mathcal{S}\left(\widehat{\wp}_{1}\right)>\mathcal{S}\left(\widehat{\wp}_{2}\right)$ then $\widehat{\wp}_{1}>\widehat{\wp}_{2}$,

3. if $\mathcal{S}\left(\widehat{\wp}_{1}\right)=\mathcal{S}\left(\widehat{\wp}_{2}\right)$ then

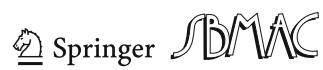


(i) if $\mathcal{A}\left(\widehat{\wp}_{1}\right)<\mathcal{A}\left(\widehat{\wp}_{2}\right)$ then $\widehat{\wp}_{1} \prec \widehat{\wp}_{2}$,

(ii) if $\mathcal{A}\left(\widehat{\wp}_{1}\right)>\mathcal{A}\left(\widehat{\wp}_{2}\right)$ then $\widehat{\wp}_{1} \succ \widehat{\wp}_{2}$,

(iii) if $\mathcal{A}\left(\widehat{\wp}_{1}\right)=\mathcal{A}\left(\widehat{\wp}_{2}\right)$ then
(a) if $\mathcal{C}\left(\widehat{\wp}_{1}\right)<\mathcal{C}\left(\widehat{\wp}_{2}\right)$ then $\widehat{\wp}_{1} \prec \widehat{\wp}_{2}$,
(b) if $\mathcal{C}\left(\widehat{\wp}_{1}\right)>\mathcal{C}\left(\widehat{\wp}_{2}\right)$ then $\widehat{\wp}_{1} \succ \widehat{\wp}_{2}$,
(c) if $\mathcal{C}\left(\widehat{\wp}_{1}\right)=\mathcal{C}\left(\widehat{\wp}_{2}\right)$ then $\widehat{\wp}_{1} \approx \widehat{\wp}_{2}$.

In above definition, we can simplify it as a binary relation $\precsim(\mathcal{S}, \mathcal{A}, \mathcal{C})$ on $\mathcal{P}$ given as

$$
\widehat{\wp}_{1} \precsim(\mathcal{S}, \mathcal{A}, \mathcal{C}) \widehat{\wp}_{2} \Leftrightarrow\left(\begin{array}{l}
\left(\mathcal{S}\left(\widehat{\wp}_{1}\right)<\mathcal{S}\left(\widehat{\wp}_{2}\right)\right) \vee\left(\left(\mathcal{S}\left(\widehat{\wp}_{1}\right)=\mathcal{S}\left(\widehat{\wp}_{2}\right)\right) \wedge\left(\mathcal{A}\left(\widehat{\wp}_{1}\right)<\mathcal{A}\left(\widehat{\wp}_{2}\right)\right)\right) \\
\vee\left(\left(\mathcal{S}\left(\widehat{\wp}_{1}\right)=\mathcal{S}\left(\widehat{\wp}_{2}\right)\right) \wedge\left(\mathcal{A}\left(\widehat{\wp}_{1}\right)=\mathcal{A}\left(\widehat{\wp}_{2}\right)\right) \wedge\left(\mathcal{C}\left(\widehat{\wp}_{1}\right) \leq \mathcal{C}\left(\widehat{\wp}_{2}\right)\right)\right)
\end{array}\right)
$$

for all $\widehat{\wp}_{1}, \widehat{\wp}_{2} \in \mathcal{P}$.

Example 2.5 Let us consider the IVPHFS $\widetilde{P}$ in Example 2.2. For the air pollution of the cities of Ankara and Istanbul, we have the following IVPHFEs:

$$
\widehat{\wp}_{1}=\{\{[0.2,0.4]\},\{[0.1,0.3],[0.2,0.4]\},\{[0,0.1],[0.1,0.2]\}\}
$$

and

$$
\widehat{\wp}_{2}=\{\{[0.1,0.3]\},\{[0.2,0.2],[0.3,0.4],[0.2,0.5]\},\{[0,0.1]\}\} .
$$

Then, we calculate as $\mathcal{S}\left(\widehat{\wp}_{1}\right)=0.475>0.425=\mathcal{S}\left(\widehat{\wp}_{2}\right)$, and so $\widehat{\wp}_{1} \succ \widehat{\wp}_{2}$.

On the other hand, we consider the IVPHFE as

$$
\widehat{\wp 3}=\{\{[0.2,0.4]\},\{[0,0.1],[0.1,0.2]\},\{[0.1,0.3],[0.2,0.4]\}\} .
$$

Then, we obtain that $\mathcal{S}\left(\widehat{\wp}_{1}\right)=\mathcal{S}\left(\widehat{\wp}_{2}\right)=0.475$ using score function and $\mathcal{A}\left(\widehat{\wp}_{1}\right)=\mathcal{A}\left(\widehat{\wp}_{2}\right)=$ 0.65 using accuracy function. Using certainty function, we have $\mathcal{C}\left(\widehat{\wp}_{1}\right)=0.6>0.525$ $=\mathcal{C}\left(\widehat{\wp}_{2}\right)$ and so $\widehat{\wp}_{1} \succ \widehat{\wp}_{2}$.

Definition 2.6 Let $\widehat{\wp}=\{\widehat{\alpha}, \widehat{\beta}, \widehat{\gamma}\}, \widehat{\wp}_{1}=\left\{\widehat{\alpha}_{1}, \widehat{\beta}_{1}, \widehat{\gamma}_{1}\right\}$ and $\widehat{\wp}_{2}=\left\{\widehat{\alpha}_{2}, \widehat{\beta}_{2}, \widehat{\gamma}_{2}\right\}$ be three IVPHFEs and $\xi$ be any real number (i.e., $\xi>0$ ). Then, the operations of IVPHFEs are described as

(a)

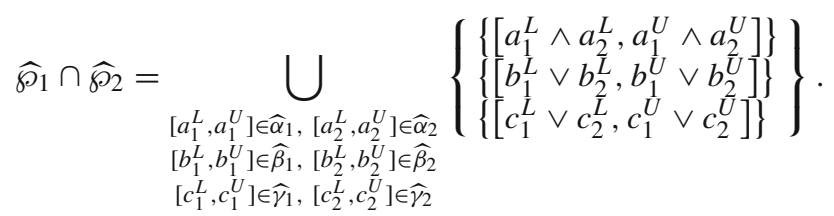

(b)

$$
\widehat{\wp}_{1} \cup \widehat{\wp}_{2}=\bigcup_{\substack{\left[a_{1}^{L}, a_{1}^{U}\right] \in \widehat{\alpha}_{1},\left[a_{2}^{L}, a_{2}^{U}\right] \in \widehat{\alpha}_{2} \\
\left[b_{1}^{L}, b_{1}^{U}\right] \in \widehat{\beta}_{1},\left[b_{2}^{L}, b_{2}^{U}\right] \in \widehat{\beta}_{2} \\
\left[c_{1}^{L}, c_{1}^{U}\right] \in \widehat{\gamma}_{1},\left[c_{2}^{L}, c_{2}^{U}\right] \in \widehat{\gamma}_{2}}}\left\{\begin{array}{l}
\left\{\left[a_{1}^{L} \vee a_{2}^{L}, a_{1}^{U} \vee a_{2}^{U}\right]\right\} \\
\left\{\left[b_{1}^{L} \wedge b_{2}^{L}, b_{1}^{U} \wedge b_{2}^{U}\right]\right\} \\
\left.\left[c_{1}^{L} \wedge c_{2}^{L}, c_{1}^{U} \wedge c_{2}^{U}\right]\right\}
\end{array}\right\} .
$$

(c)

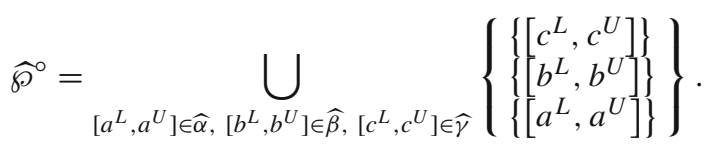


(d)

$$
\begin{aligned}
& \widehat{\wp}_{1} \oplus \widehat{\wp}_{2}=\underset{\left[a_{1}^{L}, a_{1}^{U}\right] \in \widehat{\alpha}_{1},\left[a_{2}^{L}, a_{2}^{U}\right] \in \widehat{\alpha}_{2}}{\bigcup_{\left[b_{1}\right.}^{U}}\left\{\begin{array}{l}
\left\{\left[a_{1}^{L}+a_{2}^{L}-a_{1}^{L} a_{2}^{L}, a_{1}^{U}+a_{2}^{U}-a_{1}^{U} a_{2}^{U}\right]\right\} \\
\left\{\left[b_{1}^{L} b_{2}^{L}, b_{1}^{U} b_{2}^{U}\right]\right\} \\
\left\{\left[c_{1}^{L} c_{2}^{L}, c_{1}^{U} c_{2}^{U}\right]\right\}
\end{array}\right\} . \\
& {\left[b_{1}^{L}, b_{1}^{U}\right] \in \widehat{\beta}_{1},\left[b_{2}^{L}, b_{2}^{U}\right] \in \widehat{\beta}_{2}} \\
& {\left[c_{1}^{L}, c_{1}^{U}\right] \in \widehat{\gamma}_{1},\left[c_{2}^{L}, c_{2}^{U}\right] \in \widehat{\gamma}_{2}}
\end{aligned}
$$

(e)

$$
\widehat{\wp}_{1} \otimes \widehat{\wp}_{2}=\bigcup_{\substack{\left[a_{1}^{L}, a_{1}^{U}\right] \in \widehat{\alpha}_{1},\left[a_{2}^{L}, a_{2}^{U}\right] \in \widehat{\alpha}_{2} \\
\left[b_{1}^{L}, b_{1}^{U}\right] \in \widehat{\beta}_{1},\left[b_{2}^{L}, b_{2}^{U}\right] \in \widehat{\beta}_{2} \\
\left[c_{1}^{L}, c_{1}^{U}\right] \in \widehat{\gamma}_{1},\left[c_{2}^{L}, c_{2}^{U}\right] \in \widehat{\gamma}_{2}}}\left\{\left\{\begin{array}{l}
\left\{\left[a_{1}^{L} a_{2}^{L}, a_{1}^{U} a_{2}^{U}\right]\right\}, \\
\left.\left\{\left[b_{1}^{L}+b_{2}^{L}-b_{1}^{L} b_{2}^{L}, b_{1}^{U}+b_{2}^{U}-b_{1}^{U} b_{2}^{U}\right]\right\}\right\} \\
\left.\left\{c_{1}^{L}+c_{1}^{L} c_{2}^{L}, c_{1}^{U}+c_{2}^{U}-c_{1}^{U} c_{2}^{U}\right]\right\}
\end{array}\right\} .\right.
$$

(f)

$$
\xi \widehat{\wp}=\bigcup_{\left[a^{L}, a^{U}\right] \in \widehat{\alpha},\left[b^{L}, b^{U}\right] \in \widehat{\beta}_{,\left[c^{L}, c^{U}\right] \in \widehat{\gamma}}}\left\{\begin{array}{l}
\left\{\left[1-\left(1-a^{L}\right)^{n}, 1-\left(1-a^{U}\right)^{n}\right]\right\} \\
\left\{\left[\left(b^{L}\right)^{n},\left(b^{U}\right)^{n}\right]\right\} \\
\left\{\left[\left(c^{L}\right)^{n},\left(c^{U}\right)^{n}\right]\right\}
\end{array}\right\} .
$$

(g)

$$
\widehat{\wp}^{\xi}=\bigcup_{\left[a^{L}, a^{U}\right] \in \widehat{\alpha},\left[b^{L}, b^{U}\right] \in \widehat{\beta},\left[c^{L}, c^{U}\right] \in \widehat{\gamma}}\left\{\begin{array}{l}
\left\{\left[\left(a^{L}\right)^{n},\left(a^{U}\right)^{n}\right]\right\} \\
\left\{\left[1-\left(1-b^{L}\right)^{n}, 1-\left(1-b^{U}\right)^{n}\right]\right\} \\
\left\{\left[1-\left(1-c^{L}\right)^{n}, 1-\left(1-c^{U}\right)^{n}\right]\right\}
\end{array}\right\} .
$$

Based on the aggregation principle and the operational laws of IVPHFEs, we describe some common interval-valued picture hesitant fuzzy aggregation operators.

Definition 2.7 Let $\widehat{\wp}_{j}(j=1,2, \ldots, m)$ be a collection of IVPHFEs. In addition, let $\omega=$ $\left(\omega_{1}, \omega_{2}, \ldots, \omega_{m}\right)^{T}$ be the weighted vector of $\widehat{\wp}_{j}(j=1,2, \ldots, m)$ with $\omega_{j} \in[0,1]$ and $\sum_{j=1}^{m} \omega_{j}=1$

(a) An interval-valued picture hesitant fuzzy weighted average (IVPHFWA) operator is a mapping IVPHFW $A_{\omega}: \mathcal{P}^{m} \rightarrow \mathcal{P}$, such that

$$
I V P H F W A_{\omega}\left(\widehat{\wp}_{1}, \widehat{\wp}_{2}, \ldots, \widehat{\wp}_{m}\right)=\bigoplus_{j=1}^{m} \omega_{j} \widehat{\wp}_{j}=\omega_{1} \widehat{\wp}_{1} \oplus \omega_{2} \widehat{\oslash}_{2} \oplus \ldots \oplus \omega_{m} \widehat{\wp}_{m}
$$

(b) An interval-valued picture hesitant fuzzy weighted geometric (IVPHFWG) operator is a mapping IVPHFWG $G_{\omega}: \mathcal{P}^{m} \rightarrow \mathcal{P}$, such that

$$
I V P H F W G_{\omega}\left(\widehat{\wp}_{1}, \widehat{\wp}_{2}, \ldots, \widehat{\wp}_{m}\right)=\bigotimes_{j=1}^{m} \widehat{\wp}_{j}^{\omega_{j}}=\widehat{\wp}_{1}^{\omega_{1}} \otimes \widehat{\wp}_{2}^{\omega_{2}} \otimes \ldots \otimes \widehat{\wp}_{m}^{\omega_{m}}
$$

Theorem 2.8 Let $\widehat{\wp}_{j}(j=1,2, \ldots, m)$ be a collection of IVPHFEs. In addition, let $\omega=$ $\left(\omega_{1}, \omega_{2}, \ldots, \omega_{m}\right)^{T}$ be the weighted vector of $\widehat{\wp}_{j}(j=1,2, \ldots, m)$ with $\omega_{j} \in[0,1]$ and $\sum_{j=1}^{m} \omega_{j}=1$

晋 Springer SB/ 
1. The aggregation value of IVPHFEs using the IVPHFWA operator is also an IVPHFE, and

IV P H FW $A_{\omega}\left(\widehat{\wp}_{1}, \widehat{\wp 2}, \ldots, \widehat{\wp} m\right)$

$$
=\bigoplus_{j=1}^{m} \omega_{j} \widehat{\wp}_{j}=\underbrace{\bigcup}_{\substack{\left[a_{j}^{L}, a_{j}^{U}\right] \in \widehat{\alpha}_{j}(j=1,2, \ldots, m) \\
\left[b_{j}^{L}, b_{j}^{U}\right] \in \widehat{\beta}_{j}(j=1,2, \ldots, m) \\
\left[c_{j}^{L}, c_{j}^{U}\right] \in \widehat{\gamma}_{j}(j=1,2, \ldots, m)}}\left\{\left\{\left[\begin{array}{l}
\left.\left[1-\prod_{j=1}^{m}\left(1-a_{j}^{L}\right)^{\omega_{j}}, 1-\prod_{j=1}^{m}\left(1-a_{j}^{U}\right)^{\omega_{j}}\right]\right\} \\
\left.\left.\prod_{j=1}^{m}\left(b_{j}^{L}\right)^{\omega_{j}}, \prod_{j=1}^{m}\left(b_{j}^{U}\right)^{\omega_{j}}\right]\right\}
\end{array}\right\} .\right.\right.
$$

2. The aggregation value of IVPHFEs using the IVPHFWG operator is also an IVPHFE, and

$$
\begin{aligned}
& I V P H F W G_{\omega}\left(\widehat{\wp}_{1}, \widehat{\wp}_{2}, \ldots, \widehat{\wp}_{m}\right)
\end{aligned}
$$

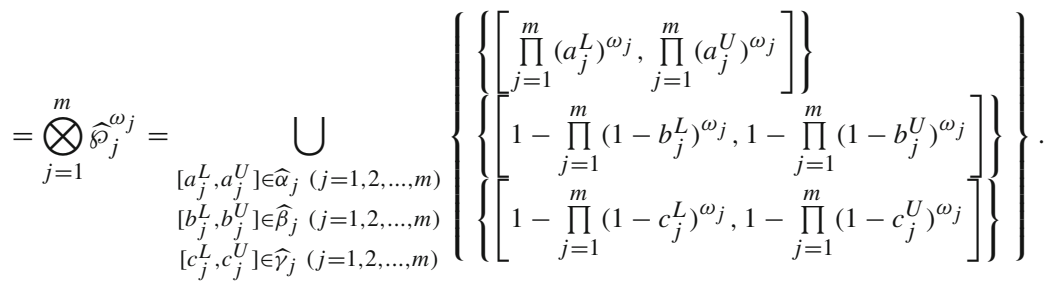

Proof The proofs are similar to the proof of Theorem 3.6, so the repetition is avoided.

Example 2.9 We consider $\widehat{\wp}_{1}$ and $\widehat{\wp}_{2}$ given in Example 2.5. From Example 2.2, we have the following IVPHFEs for the air pollution of the cities of Yozgat and Antalya, respectively:

$$
\widehat{\wp} 3=\{\{[0.2,0.35],[0.3,0.4]\},\{[0.1,0.25],[0.2,0.3]\},\{[0.1,0.2]\}\}
$$

and

$$
\widehat{\wp}_{4}=\{\{[0.1,0.3],[0.2,0.5]\},\{[0.2,0.2]\},\{[0.1,0.1]\}\} .
$$

Then, we have

$$
\begin{array}{r}
\widehat{\wp}_{1} \cap \widehat{\wp}_{4}=\{\{[0.1,0.3],[0.2,0.4]\},\{[0.2,0.3],[0.2,0.4]\},\{[0.1,0.1],[0.1,0.2]\}\}, \\
\widehat{\wp}_{1} \cup \widehat{\wp}_{4}=\{\{[0.2,0.4],[0.2,0.5]\},\{[0.1,0.2],[0.2,0.2]\},\{[0,0.1],[0.1,0.1]\}\}, \\
\widehat{\wp}_{3}^{\circ}=\{\{[0.1,0.2]\},\{[0.1,0.25],[0.2,0.3]\},\{[0.2,0.35],[0.3,0.4]\}\},
\end{array}
$$

Assume that $\omega=\left(\omega_{1}, \omega_{2}, \omega_{3}\right)=(0.2,0.3,0.5)^{T}$ for $\widehat{\wp}_{1}, \widehat{\wp}_{2}$ and $\widehat{\wp}_{3}$. Then, we obtain

$$
\left.\begin{array}{l}
I V P H F W A_{\omega}\left(\widehat{\wp}_{1}, \widehat{\wp}_{2}, \widehat{\wp}_{3}\right)=\bigoplus_{j=1}^{3} \omega_{j} \widehat{\wp}_{j} \\
=\left\{\begin{array}{l}
\{[0.1712,0.3459],[0.2247,0.3716]\}, \\
{[0.1213,0.2424],[0.1741,0.3067],[0.139,0.2985],[0.1966,0.3776],} \\
{[0.1597,0.3162],[0.2258,0.4],[0.1414,0.3381],[0.2,0.4276]} \\
[0,0.1414],[0,0.1624]\}
\end{array}\right\},
\end{array}\right\},
$$


and

$$
\left.\begin{array}{l}
I V P H F W G_{\omega}\left(\widehat{\wp}_{1}, \widehat{\wp}_{2}, \widehat{\wp}_{3}\right)=\bigotimes_{j=1}^{3} \widehat{\wp}_{j}^{\omega_{j}} \\
=\left\{\begin{array}{l}
\{[0.1624,0.3432],[0.1989,0.3669]\}, \\
{[0.1312,0.2458],[0.1809,0.3254],[0.1653,0.3081],[0.213,0.3812],} \\
{[0.1312,0.345],[0.1809,0.4141],[0.1514,0.2687],[0.2,0.3459],} \\
{[0.1847,0.3291],[0.2314,0.4],[0.1514,0.3648],[0.2,0.4319]}
\end{array}\right\},
\end{array}\right\} .
$$

All the operations, relations, and operators of IVPHFEs can only be used to deal with timeindependent IVPHF arguments. However, these notions are not satisfactory if the IVPHF information may be collected at the different periods, where the period duration is a point or portion of the time.

\section{Dynamic interval-valued picture hesitant fuzzy weighted aggregation operators}

In this part, we define some dynamic interval-valued picture hesitant fuzzy weighted aggregation operators to aggregate interval-valued picture hesitant fuzzy information collected at the different periods, where the period duration is a point or portion of the time.

Definition 3.1 Let $t$ be the variable of time, then we define an interval-valued picture hesitant fuzzy variable (IVPHFV) as $\widehat{\wp}(t)=\{\widehat{\alpha}(t), \widehat{\beta}(t), \widehat{\gamma}(t)\}=$

$\left\{\left\{\left[a_{(t)}^{L}, a_{(t)}^{U}\right]\right\},\left\{\left[b_{(t)}^{L}, b_{(t)}^{U}\right]\right\},\left\{\left[c_{(t)}^{L}, c_{(t)}^{U}\right]\right\}\right\}$.

$\left[a_{(t)}^{L}, a_{(t)}^{U}\right] \in \widehat{\alpha}_{(t)},\left[b_{(t)}^{L}, b_{(t)}^{U}\right] \in \widehat{\beta}_{(t)},\left[c_{(t)}^{L}, c_{(t)}^{U}\right] \in \widehat{\gamma}_{(t)}$

For an IVPHFV $\widehat{\wp}(t)=\{\widehat{\alpha}(t), \widehat{\beta}(t), \widehat{\gamma}(t)\}$, if $t=t_{1}, t_{2}, \ldots, t_{k}$ then $\widehat{\wp}\left(t_{1}\right), \widehat{\wp}\left(t_{2}\right), \ldots, \widehat{\wp}\left(t_{k}\right)$ is represented as $k$ interval-valued picture hesitant fuzzy elements collected at $k$ periods. Now, we introduce some operations related to the values of IVPHFVs.

Definition 3.2 Let $\widehat{\wp}\left(t_{1}\right)=\left\{\widehat{\alpha}\left(t_{1}\right), \widehat{\beta}\left(t_{1}\right), \widehat{\gamma}\left(t_{1}\right)\right\}$ and $\widehat{\wp}\left(t_{2}\right)=\left\{\widehat{\alpha}\left(t_{2}\right), \widehat{\beta}\left(t_{2}\right), \widehat{\gamma}\left(t_{2}\right)\right\}$ be two IVPHFVs and $\xi$ be any positive real number (i.e., $\xi>0$ ). Then, the operations of IVPHFVs are described as

(a)

$$
\begin{aligned}
& \widehat{\wp}\left(t_{1}\right) \oplus \widehat{\wp}\left(t_{2}\right)
\end{aligned}
$$

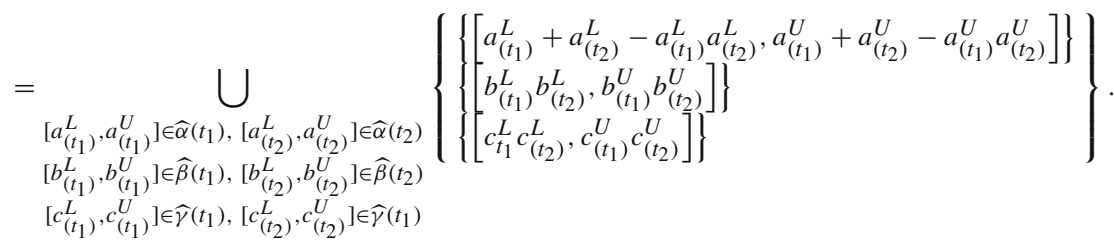

(b)

$$
\widehat{\wp}\left(t_{1}\right) \otimes \widehat{\wp}\left(t_{2}\right)
$$




$$
\begin{aligned}
& =\underset{\left[a_{\left(t_{1}\right)}^{L}, a_{\left(t_{1}\right)}^{U}\right] \in \widehat{\alpha}\left(t_{1}\right),\left[a_{\left(t_{2}\right)}^{L}, a_{\left(t_{2}\right)}^{U}\right] \in \widehat{\alpha}\left(t_{2}\right)}{\bigcup}\left\{\left\{\left\{\begin{array}{l}
\left.\left[a_{\left(t_{1}\right)}^{L} a_{\left(t_{2}\right)}^{L}, a_{\left(t_{1}\right)}^{U} a_{\left(t_{2}\right)}^{U}\right]\right\} \\
\left.\left.b_{\left(t_{1}\right)}^{L}+b_{\left(t_{2}\right)}^{L}-b_{\left(t_{1}\right)}^{L} b_{\left(t_{2}\right)}^{L}, b_{\left(t_{1}\right)}^{U}+b_{\left(t_{2}\right)}^{U}-b_{\left(t_{1}\right)}^{U} b_{\left(t_{2}\right)}^{U}\right]\right\} \\
\left.\left.c_{\left(t_{1}\right)}^{L}+c_{\left(t_{2}\right)}^{L}-c_{\left(t_{1}\right)}^{L} c_{\left(t_{2}\right)}^{L}, c_{\left(t_{1}\right)}^{U}+c_{\left(t_{2}\right)}^{U}-c_{\left(t_{1}\right)}^{U} c_{\left(t_{2}\right)}^{U}\right]\right\}
\end{array}\right\} .\right.\right. \\
& {\left[b_{\left(t_{1}\right)}^{L}, b_{\left(t_{1}\right)}^{U}\right] \in \widehat{\beta}\left(t_{1}\right),\left[b_{\left(t_{2}\right)}^{L}, b_{\left(t_{2}\right)}^{U}\right] \in \widehat{\beta}\left(t_{2}\right)} \\
& {\left[c_{\left(t_{1}\right)}^{L}, c_{\left(t_{1}\right)}^{U}\right] \in \widehat{\gamma}\left(t_{1}\right),\left[c_{\left(t_{2}\right)}^{L}, c_{\left(t_{2}\right)}^{U}\right] \in \widehat{\gamma}\left(t_{2}\right)}
\end{aligned}
$$

(c)

$$
\xi \widehat{\wp}\left(t_{1}\right)=\bigcup_{\substack{\left[a_{\left(t_{1}\right)}^{L}, a_{\left(t_{1}\right)}^{U}\right] \in \widehat{\alpha}_{\left(t_{1}\right)} \\
\left[b_{\left(t_{1}\right)}^{L}, b_{\left(t_{1}\right)}^{U}\right] \in \widehat{\beta}\left(t_{1}\right) \\
\left[c_{\left(t_{1}\right)}^{L}, c_{\left(t_{1}\right)}^{U}\right] \in \widehat{\gamma}\left(t_{1}\right)}}\left\{\left\{\left\{\left[\begin{array}{l}
\left.\left.\left[b_{\left(t_{1}\right)}^{L}\right)^{n},\left(b_{\left(t_{1}\right)}^{U}\right)^{n}\right]\right\} \\
\left.\left.\left(c_{\left(t_{1}\right)}^{L}\right)^{n},\left(c_{\left(t_{1}\right)}^{U}\right)^{n}\right]\right\}
\end{array}\right\} .\right.\right.\right.
$$

(d)

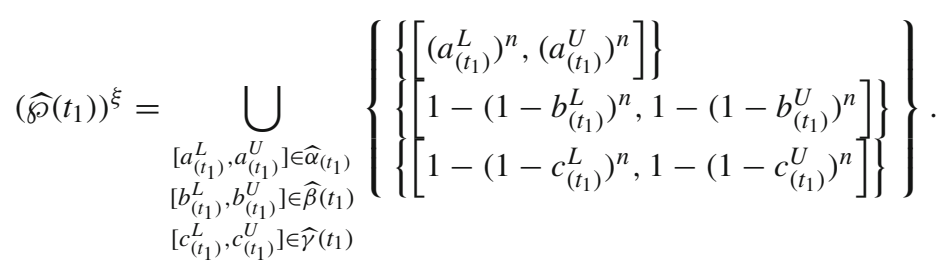

Example 3.3 We consider Example 2.2. For the city of Yozgat, let us assume that the air pollution is evaluated according to the interval-valued picture hesitant fuzzy information collected in two different periods. According to the two-period evaluations, we consider two following IVPHFEs:

For the period $t_{1}$,

$$
\widehat{\wp}\left(t_{1}\right)=\{\{[0.2,0.35],[0.3,0.4]\},\{[0.1,0.25],[0.2,0.3]\},\{[0.1,0.2]\}\}
$$

and for the period $t_{2}$,

$$
\widehat{\wp}\left(t_{2}\right)=\{\{[0.1,0.15]\},\{[0.1,0.2],[0.15,0.3],[0.2,0.4]\},\{[0,0.1]\}\} .
$$

In addition, let $\xi=4$. Then, we obtain

$\widehat{\wp}\left(t_{1}\right) \oplus \widehat{\wp}\left(t_{2}\right)=\{\{[0.28,0.4475],[0.37,0.49]\},\{[0.01,0.05],[0.015,0.075],[0.02,0.1]$,

[0.02, 0.06], [0.03, 0.09], [0.04, 0.12]\}, \{[0, 0.02]\}\},

$\widehat{\wp}\left(t_{1}\right) \otimes \widehat{\wp}\left(t_{2}\right)=\{\{[0.02,0.0525],[0.03,0.06]\},\{[0.19,0.4],[0.235,0.475],[0.28,0.55]$,

$[0.28,0.44],[0.32,0.51],[0.36,0.58]\},\{[0.1,0.28]\}\}$,

$4 \widehat{\wp}\left(t_{1}\right)=\{\{[0.5904,0.8215],[0.76,0.8704]\},\{[0.0001,0.0039],[0.0016,0.0081]\},\{[0.0001,0.0016]\}\}$,

$\widehat{\wp}\left(t_{2}\right)^{4}=\{\{[0.0001,0.0005]\},\{[0.3439,0.4096],[0.478,0.76],[0.4096,0.8704]\},\{[0,0.3439]\}\}$.

Proposition 3.4 Let $\widehat{\wp}\left(t_{1}\right)=\left\{\widehat{\alpha}\left(t_{1}\right), \widehat{\beta}\left(t_{1}\right), \widehat{\gamma}\left(t_{1}\right)\right\}$ and $\widehat{\wp}\left(t_{2}\right)=\left\{\widehat{\alpha}\left(t_{2}\right), \widehat{\beta}\left(t_{2}\right), \widehat{\gamma}\left(t_{2}\right)\right.$ be two IVPHFVs and $\xi>0$.

1. $\widehat{\wp}\left(t_{1}\right) \oplus \widehat{\wp}\left(t_{2}\right)=\widehat{\wp}\left(t_{2}\right) \oplus \widehat{\wp}\left(t_{1}\right)$.

2. $\widehat{\wp}\left(t_{1}\right) \otimes \widehat{\wp}\left(t_{2}\right)=\widehat{\wp}\left(t_{2}\right) \otimes \widehat{\wp}\left(t_{1}\right)$.

3. $\xi\left(\widehat{\wp}\left(t_{1}\right) \oplus \widehat{\varnothing}\left(t_{2}\right)\right)=\xi \widehat{\varnothing}\left(t_{1}\right) \oplus \xi \widehat{\varnothing}\left(t_{2}\right)$.

4. $\left(\widehat{\wp}\left(t_{1}\right) \otimes \widehat{\wp}\left(t_{2}\right)\right)^{\xi}=\left(\widehat{\wp}\left(t_{1}\right)\right)^{\xi} \otimes\left(\widehat{\wp}\left(t_{2}\right)\right)^{\xi}$. 
Proof It is trivial from Definition 3.2.

By combining the above operations described for IVPHFVs, the following weighted aggregation operators are developed.

Definition 3.5 Let $\widehat{\wp}\left(t_{k}\right)(k=1,2, \ldots, r)$ be the IVPHFEs collected from $k$ different periods $t_{k}(k=1,2, \ldots, r)$. In addition, let $\omega(t)=\left(\omega\left(t_{1}\right), \omega\left(t_{2}\right), \ldots, \omega\left(t_{r}\right)\right)^{T}$ be the weighted vector of $t_{k}(k=1,2, \ldots, r)$, with $\omega\left(t_{k}\right) \in[0,1]$ and $\sum_{k=1}^{r} \omega\left(t_{k}\right)=1$, and $\lambda>0$.

(a) A dynamic interval-valued picture hesitant fuzzy weighted average (DIVPHFWA) operator is a mapping DIVPHFW $A_{\omega(t)}: \mathcal{P}^{r} \rightarrow \mathcal{P}$, such that

$$
\begin{gathered}
D I V P H F W A_{\omega(t)}\left(\widehat{\wp}\left(t_{1}\right), \widehat{\wp}\left(t_{2}\right), \ldots, \widehat{\wp}\left(t_{r}\right)\right)=\bigoplus_{k=1}^{r} \omega\left(t_{k}\right) \widehat{\wp}\left(t_{k}\right) \\
=\omega\left(t_{1}\right) \widehat{\wp}\left(t_{1}\right) \oplus \omega\left(t_{2}\right) \widehat{\wp}\left(t_{2}\right) \oplus \ldots \oplus \omega\left(t_{r}\right) \widehat{\wp}\left(t_{r}\right)
\end{gathered}
$$

(b) A dynamic interval-valued picture hesitant fuzzy weighted geometric (DIVPHFWG) operator is a mapping $\operatorname{DIVPHFWG} G_{\omega(t)}: \mathcal{P}^{r} \rightarrow \mathcal{P}$, such that

$$
\begin{gathered}
D I V P H F W G_{\omega(t)}\left(\widehat{\wp}\left(t_{1}\right), \widehat{\wp}\left(t_{2}\right), \ldots, \widehat{\wp}\left(t_{r}\right)\right)=\bigotimes_{k=1}^{r}\left(\widehat{\wp}\left(t_{k}\right)\right)^{\omega\left(t_{k}\right)} \\
=\left(\widehat{\wp}\left(t_{1}\right)\right)^{\omega\left(t_{1}\right)} \otimes\left(\widehat{\wp}\left(t_{2}\right)\right)^{\omega\left(t_{2}\right)} \otimes \ldots \otimes\left(\widehat{\wp}\left(t_{k}\right)\right)^{\omega\left(t_{k}\right)}
\end{gathered}
$$

(c) A generalized dynamic interval-valued picture hesitant fuzzy weighted average (GDIVPHFWA) operator is a mapping GDIVPHFW $A_{\omega(t)}: \mathcal{P}^{r} \rightarrow \mathcal{P}$ such that

$$
\begin{gathered}
\text { GDIVP HFW } A_{\omega(t)}\left(\widehat{\wp}\left(t_{1}\right), \widehat{\wp}\left(t_{2}\right), \ldots, \widehat{\wp}\left(t_{r}\right)\right)=\left(\bigoplus_{k=1}^{r} \omega\left(t_{k}\right)\left(\widehat{\wp}\left(t_{k}\right)\right)^{\lambda}\right)^{\frac{1}{\lambda}} \\
=\left(\omega\left(t_{1}\right)\left(\widehat{\wp}\left(t_{1}\right)\right)^{\lambda} \oplus \omega\left(t_{2}\right)\left(\widehat{\wp}\left(t_{2}\right)\right)^{\lambda} \oplus \ldots \oplus \omega\left(t_{r}\right)\left(\widehat{\wp}\left(t_{r}\right)\right)^{\lambda}\right)^{\frac{1}{\lambda}}
\end{gathered}
$$

(d) A generalized dynamic interval-valued picture hesitant fuzzy weighted geometric (GDIVPHFWG) operator is a mapping GDIVPHFWG $G_{\omega(t)}: \mathcal{P}^{r} \rightarrow \mathcal{P}$ such that

$$
\begin{gathered}
G D I V P H F W G_{\omega(t)}\left(\widehat{\wp}\left(t_{1}\right), \widehat{\wp}\left(t_{2}\right), \ldots, \widehat{\wp}\left(t_{r}\right)\right)=\frac{1}{\lambda}\left(\bigotimes_{k=1}^{r} \lambda\left(\widehat{\wp}\left(t_{k}\right)\right)^{\omega\left(t_{k}\right)}\right) \\
=\frac{1}{\lambda}\left(\lambda\left(\widehat{\wp}\left(t_{1}\right)\right)^{\omega\left(t_{1}\right)} \otimes \lambda\left(\widehat{\wp}\left(t_{2}\right)\right)^{\omega\left(t_{2}\right)} \otimes \ldots \otimes \lambda\left(\widehat{\wp}\left(t_{r}\right)\right)^{\omega\left(t_{r}\right)}\right)
\end{gathered}
$$

Theorem 3.6 Let $\widehat{\wp}\left(t_{k}\right)(k=1,2, \ldots, r)$ be the IVPHFEs collected from $k$ different periods $t_{k}(k=1,2, \ldots, r)$. In addition, let $\omega(t)=\left(\omega\left(t_{1}\right), \omega\left(t_{2}\right), \ldots, \omega\left(t_{r}\right)\right)^{T}$ be the weighted vector of $t_{k}(k=1,2, \ldots, r)$, with $\omega\left(t_{k}\right) \in[0,1]$ and $\sum_{k=1}^{r} \omega\left(t_{k}\right)=1$, and $\lambda>0$.

1. The aggregation value of IVPHFEs using the DIVPHFWA operator is also an IVPHFE, and

$$
\begin{aligned}
& D I V P H F W A_{\omega(t)}\left(\widehat{\wp}\left(t_{1}\right), \widehat{\wp}\left(t_{2}\right), \ldots, \widehat{\wp}\left(t_{r}\right)\right)
\end{aligned}
$$

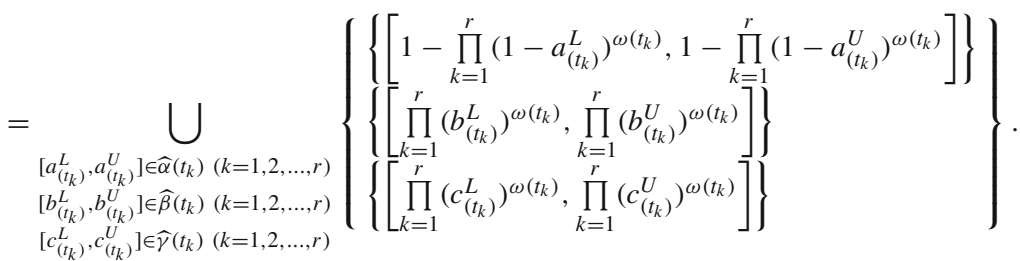


2. The aggregation value of IVPHFEs using the DIVPHFWG operator is also an IVPHFE, and

$$
\begin{aligned}
& D I V P H F W G_{\omega(t)}\left(\widehat{\wp}\left(t_{1}\right), \widehat{\wp}\left(t_{2}\right), \ldots, \widehat{\wp}\left(t_{r}\right)\right)
\end{aligned}
$$

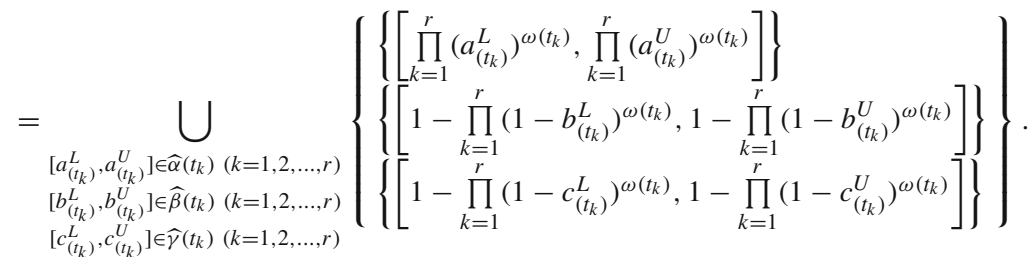

3. The aggregation value of IVPHFEs using the GDIVPHFWA operator is also an IVPHFE, and

$$
\begin{aligned}
& \operatorname{GDIVPHFWA} A_{\omega(t)}\left(\widehat{\wp}\left(t_{1}\right), \widehat{\wp}\left(t_{2}\right), \ldots, \widehat{\wp}\left(t_{r}\right)\right)
\end{aligned}
$$

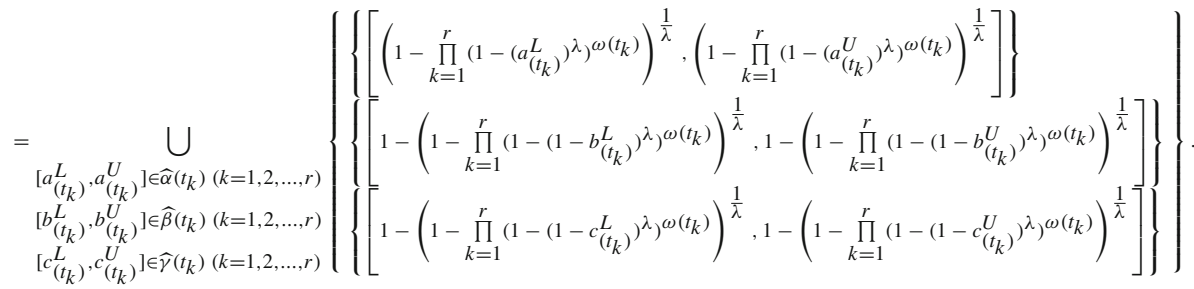

(4) The aggregation value of IVPHFEs using the GDIVPHFWG operator is also an IVPHFE, and

GDIVPHFWG $G_{\omega(t)}\left(\widehat{\wp}\left(t_{1}\right), \widehat{\wp}\left(t_{2}\right), \ldots, \widehat{\wp}\left(t_{r}\right)\right)$

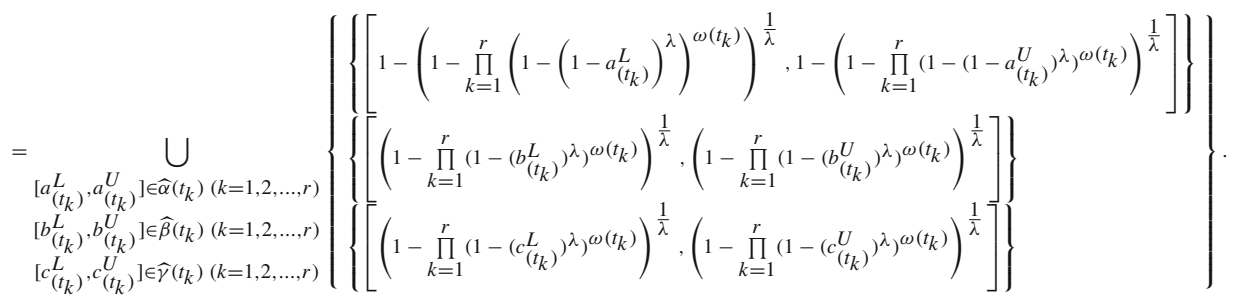

Proof Let us prove (4), the others can be demonstrate similar to this.

We use the method of mathematical induction for proof.

- For $r=1$ :

By Definition 3.2 (d), we calculate

$$
\lambda \widehat{\wp}\left(t_{1}\right)=\bigcup_{\substack{\left[a_{\left(t_{1}\right)}^{L}, a_{\left(t_{1}\right)}^{U} \\
\left[b_{\left(t_{1}\right)}^{L}, b_{\left(t_{1}\right)}^{U}\right] \in \widehat{\beta}\left(t_{1}\right) \\
\left[c_{\left(t_{1}\right)}^{L}, c_{\left(t_{1}\right)}^{U}\right] \in \widehat{\gamma}\left(t_{1}\right)\right.}}^{\bigcup_{\left(t_{1}\right)}^{U}}\left\{\begin{array}{l}
\left.\left\{\left(b_{\left(t_{1}\right)}^{L}\right)^{\lambda},\left(b_{\left(t_{1}\right)}^{U}\right)^{\lambda}\right]\right\} \\
\left.\left[\left(c_{\left(t_{1}\right)}^{L}\right)^{\lambda},\left(c_{\left(t_{1}\right)}^{U}\right)^{\lambda}\right]\right\}
\end{array}\right\}
$$


and moreover

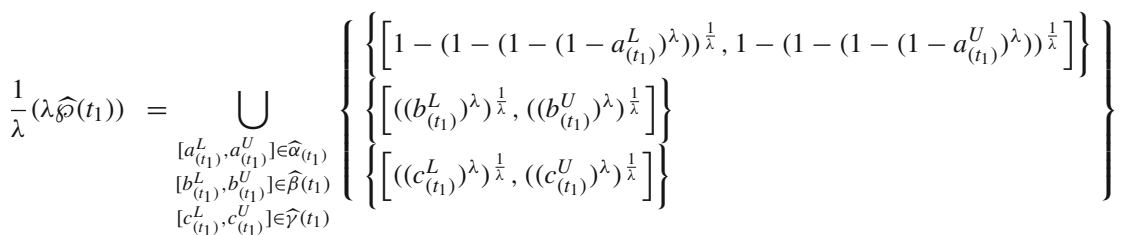

$$
\begin{aligned}
& =\bigcup_{\substack{\left[a_{(t)}, a_{(t)}^{U}\right] \in \widehat{\alpha}_{\left(t_{1}\right)} \\
\left[b_{\left(t_{1}\right)}^{L}, b_{\left(t_{1}\right)}^{U}\right] \in \widehat{\beta}\left(t_{1}\right) \\
\left[c_{\left(t_{1}\right)}^{L}, c_{\left(t_{1}\right)}^{U}\right] \in \widehat{\gamma}\left(t_{1}\right)}}\left\{\left\{\left\{\left[b_{\left(t_{1}\right)}^{L}, b_{\left(t_{1}\right)}^{U}\right]\right\}\right\}\right. \text {. }
\end{aligned}
$$

Hence, we have GDIVPHFWG $G_{\omega(t)}\left(\widehat{\wp}\left(t_{1}\right)\right)=\frac{1}{\lambda}\left(\left(\lambda \widehat{\wp}\left(t_{1}\right)\right)^{\omega\left(t_{1}\right)}\right)=\widehat{\wp}\left(t_{1}\right)$ and so Eq. (3.12) is valid for $r=1$.

- For $r=2$ :

Since (from Definition 2.6 (c) and (d))

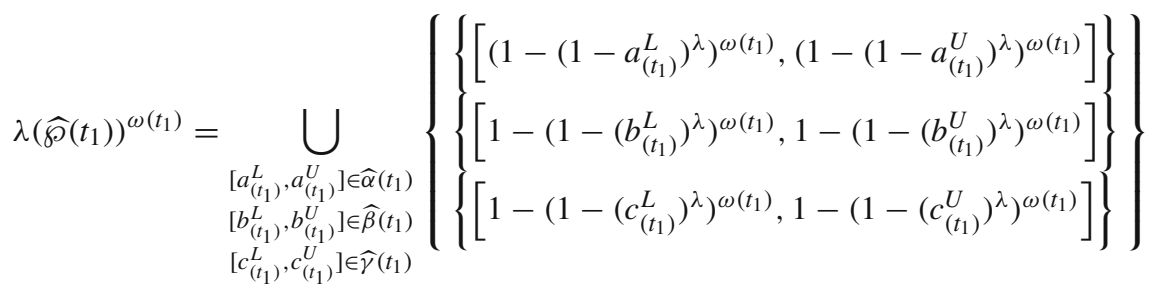

and

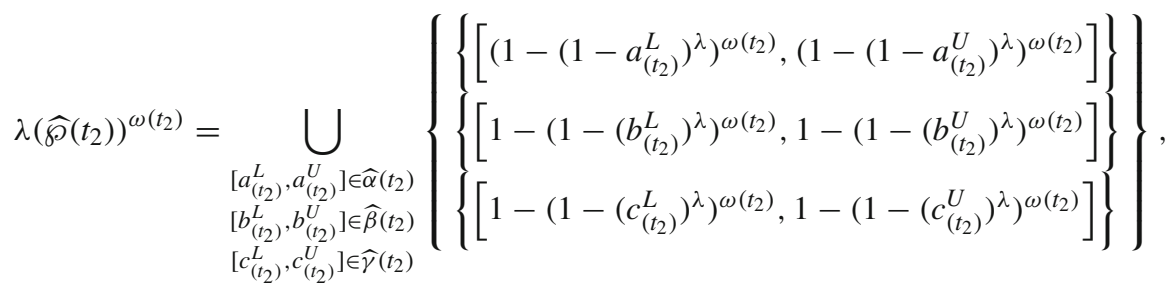

we found that (from Definition 2.6 (b))

$$
\begin{aligned}
& \lambda\left(\widehat{\wp}\left(t_{1}\right)\right)^{\omega\left(t_{1}\right)} \otimes \lambda\left(\widehat{\wp}\left(t_{2}\right)\right)^{\omega\left(t_{2}\right)}
\end{aligned}
$$

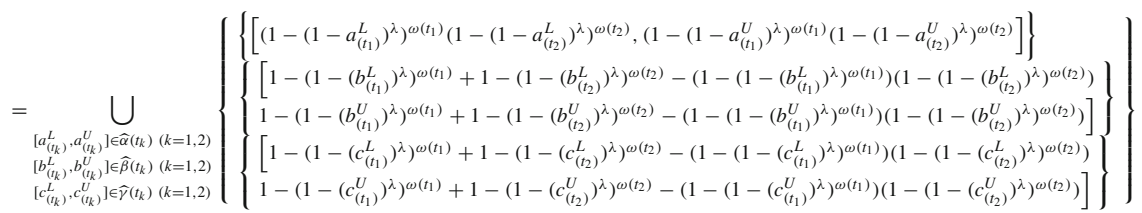

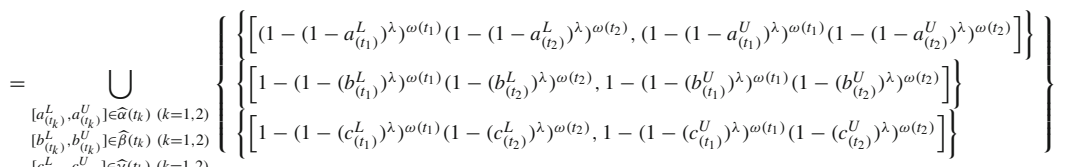




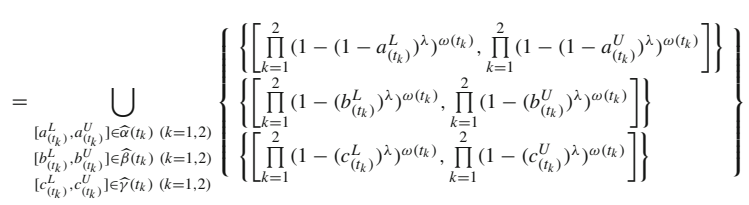

and thus

$$
\begin{aligned}
& \frac{1}{\lambda}\left(\lambda\left(\widehat{\wp}\left(t_{1}\right)\right)^{\omega\left(t_{1}\right)} \otimes \lambda\left(\widehat{\wp}\left(t_{1}\right)\right)^{\omega\left(t_{1}\right)}\right)
\end{aligned}
$$

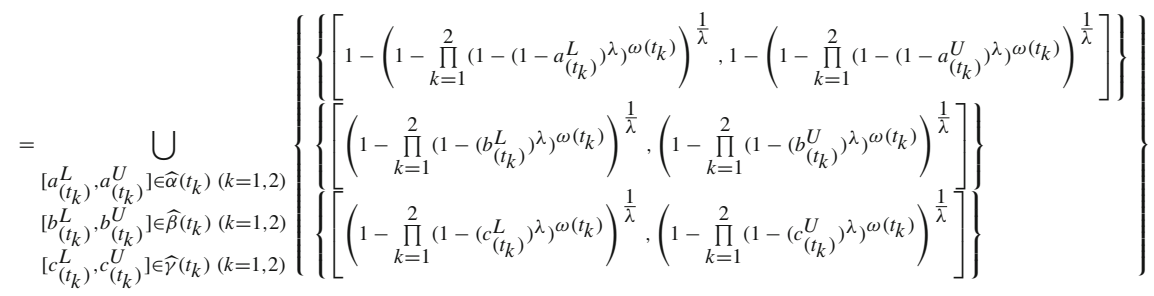

$$
\begin{aligned}
& =G D I V P H F W G_{\epsilon}^{\omega(t)}\left(\widehat{\S}\left(t_{1}\right), \widehat{\S}\left(t_{2}\right)\right) \text {. }
\end{aligned}
$$

Hence, Eq. (3.12) holds for $r=2$.

- For $r=s$ :

If Eq. (3.12) is valid, then we obtain the following

$$
\begin{aligned}
& \operatorname{GDIVPHFWG}_{\omega(t)}\left(\widehat{\wp}\left(t_{1}\right), \widehat{\S}\left(t_{2}\right), \ldots, \widehat{\varnothing}\left(t_{s}\right)\right)
\end{aligned}
$$

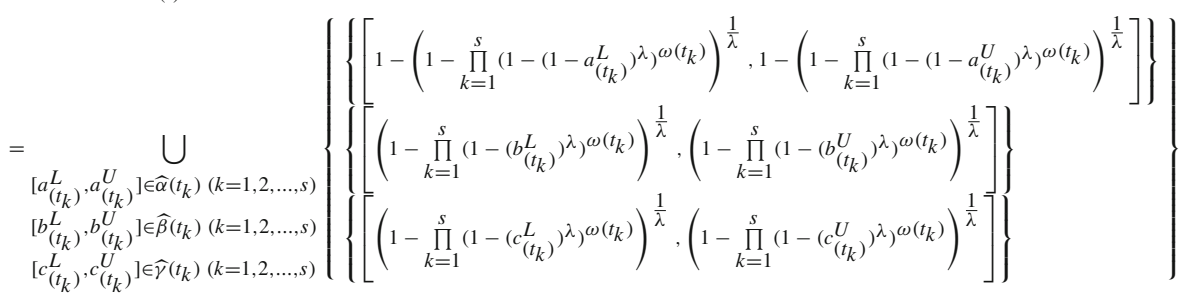

Now let's demonstrate that Eq. (3.12) holds when $r=s+1$.

In Eq. (3.13), $s$ is substituted for 2 and also by Eqs. (3.3), (3.4), we write $\lambda\left(\widehat{\wp}\left(t_{1}\right)\right)^{\omega_{1}} \otimes \lambda\left(\widehat{\wp}\left(t_{2}\right)\right)^{\omega_{2}} \otimes \ldots \otimes \lambda\left(\widehat{\wp}\left(t_{s}\right)\right)^{\omega_{s}} \otimes \lambda\left(\widehat{\wp}\left(t_{s+1}\right)\right)^{\omega_{s+1}}$

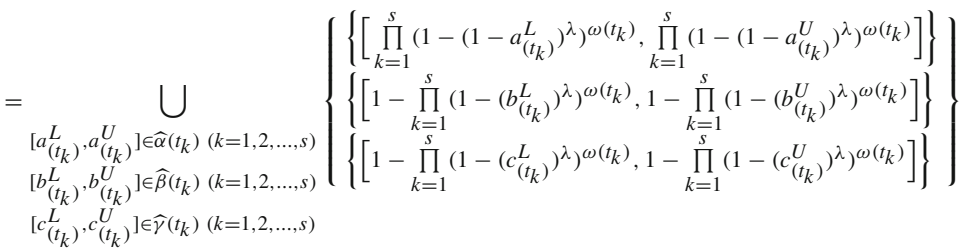

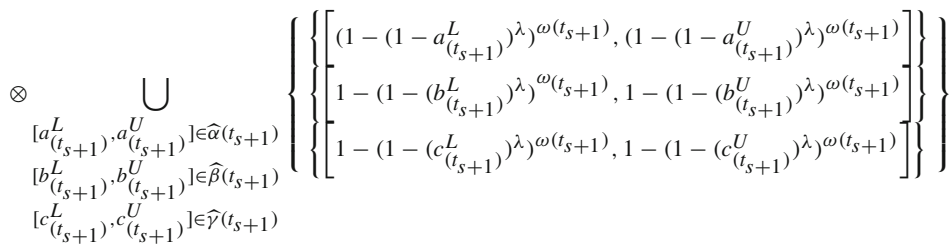




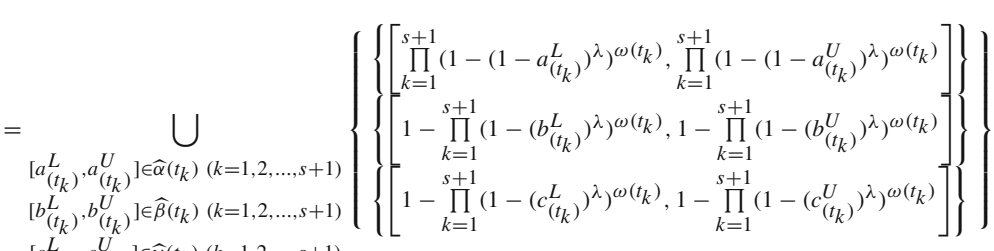

$$
\begin{aligned}
& {\left[c_{\left(t_{k}\right)}^{L}, c_{\left(t_{k}\right)}^{U}\right] \in \widehat{\gamma}\left(t_{k}\right)(k=1,2, \ldots, s+1)}
\end{aligned}
$$

Then we obtain that (from Definition 3.2 (c))

$$
\begin{aligned}
& \frac{1}{\lambda}\left(\lambda\left(\widehat{\wp}\left(t_{1}\right)\right)^{\omega\left(t_{1}\right)} \otimes \lambda\left(\widehat{\wp}\left(t_{2}\right)\right)^{\omega\left(t_{2}\right)} \otimes \ldots \otimes \lambda(\widehat{\wp})\left(t_{s}\right)^{\omega_{\left(t_{s}\right)}} \otimes \lambda\left(\widehat{\wp}\left(t_{s+1}\right)^{\omega\left(t_{s+1}\right)}\right)\right.
\end{aligned}
$$

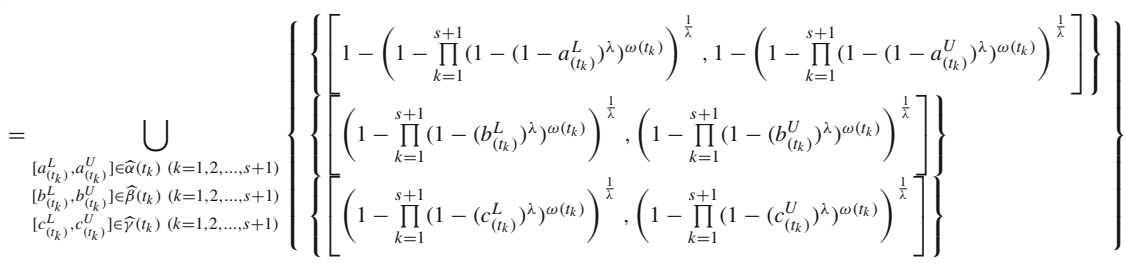

$$
\begin{aligned}
& =G D I V P H F W G_{\omega(t)}\left(\widehat{\wp}\left(t_{1}\right), \widehat{\wp}\left(t_{2}\right), \ldots, \widehat{\wp}\left(t_{s+1}\right)\right)
\end{aligned}
$$

Hence, Eq. (3.12) is valid for $r=s+1$.

Thus, we conclude that Eq. (3.12) is true for all values of $r$.

Note 2. If $\lambda=1$ then GDIVPHFWA operator and GDIVPHFWG operator reduce to DIVPHFWA operator and DIVPHFWG operator, respectively. Therefore, we will investigate the results of GDIPHFWA and GDIVPHFWG operators.

Theorem 3.7 (Idempotency) Let $\widehat{\wp}\left(t_{k}\right)(k=1,2, \ldots, r)$ be the IVPHFEs collected from $k$ different periods $t_{k}(k=1,2, \ldots, r)$. If $\widehat{\wp}\left(t_{k}\right)=\widehat{\wp}=\{\widehat{\alpha}, \widehat{\beta}, \widehat{\gamma}\}$ for all $k=1,2, \ldots, r$ then

(1) GDIVPHFWA $A_{\omega(t)}\left(\widehat{\wp}\left(t_{1}\right), \widehat{\wp}\left(t_{2}\right), \ldots, \widehat{\wp}\left(t_{r}\right)\right)=\widehat{\wp}$.

(2) GDIVPHFW $G_{\omega(t)}\left(\widehat{\wp}\left(t_{1}\right), \widehat{\wp}\left(t_{2}\right), \ldots, \widehat{\wp}\left(t_{2}\right)\right)=\widehat{\wp}$.

Proof Let us prove GDIVPHFW $G_{\omega(t)}\left(\widehat{\wp}\left(t_{1}\right), \widehat{\wp}\left(t_{2}\right), \ldots, \widehat{\wp}\left(t_{r}\right)\right)=\widehat{\wp}$, the other can be shown similar to this.

Considering $\widehat{\wp}\left(t_{k}\right)=\widehat{\wp}=\{\widehat{\alpha}, \widehat{\beta}, \widehat{\gamma}\}$, by Theorem 3.6, we have

GDIVPHFWG $G_{\omega(t)}\left(\widehat{\wp}\left(t_{1}\right), \widehat{\varnothing}\left(t_{2}\right), \ldots, \widehat{\varnothing}\left(t_{r}\right)\right)$

$$
\begin{aligned}
& =\frac{1}{\lambda}\left(\lambda\left(\widehat{\wp}\left(t_{1}\right)\right)^{\omega\left(t_{1}\right)} \otimes \lambda\left(\widehat{\wp}\left(t_{2}\right)\right)^{\omega\left(t_{2}\right)} \otimes \ldots \otimes \lambda\left(\widehat{\wp}\left(t_{r}\right)\right)^{\omega\left(t_{r}\right)}\right)
\end{aligned}
$$

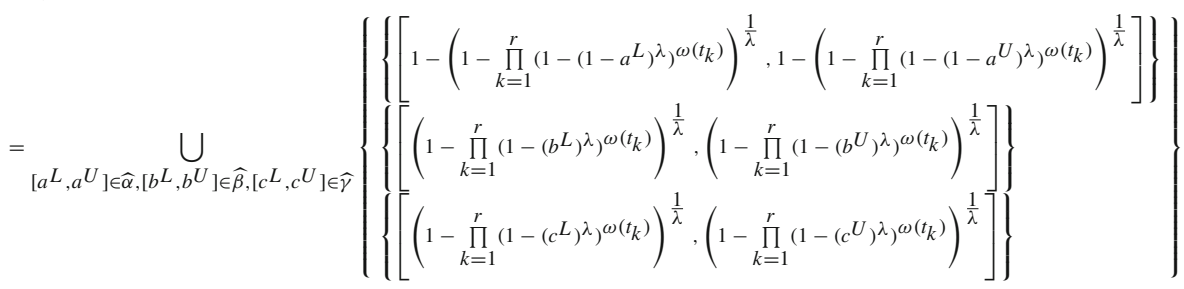

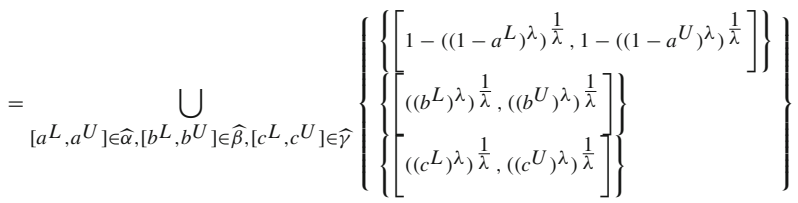

$$
\begin{aligned}
& \left.=\bigcup_{\left[a^{L}, a\right.}{ }^{U}\right] \in \widehat{\alpha},\left[b^{L}, b^{U}\right] \in{\widehat{\beta},\left[c^{L}, c^{U}\right] \in \widehat{\gamma}}_{\left\{\left[c^{L}, c^{U}\right]\right\}}\left\{\begin{array}{l}
\left\{\left[a^{L}, a^{U}\right]\right\} \\
\left.\left\{b^{L}, b^{U}\right]\right\} \\
\left\{{ }^{L}\right.
\end{array}\right\} .
\end{aligned}
$$


Thereby, the proof is completed.

Theorem 3.8 (Boundedness) Let $\widehat{\wp}\left(t_{k}\right)(k=1,2, \ldots, r)$ be the IVPHFEs collected from $k$ different periods $t_{k}(k=1,2, \ldots, r)$.

(1) $\widehat{\wp}_{\min } \leq G D I V P H F W A_{\omega(t)}\left(\widehat{\wp}\left(t_{1}\right), \widehat{\wp}\left(t_{2}\right), \ldots, \widehat{\wp}\left(t_{r}\right)\right) \leq \widehat{\wp}_{\max }$

(2) $\widehat{\wp}_{\min } \leq G D I V P H F W G_{\omega(t)}\left(\widehat{\wp}\left(t_{1}\right), \widehat{\wp}\left(t_{2}\right), \ldots, \widehat{\wp}\left(t_{r}\right)\right) \leq \widehat{\wp}_{\max }$

where

$$
\begin{aligned}
& \widehat{\wp}_{\min }=\left\{\widehat{\alpha}_{\min }, \widehat{\beta}_{\max }, \widehat{\gamma}_{\max }\right\}=\left\{\left\{\left[a_{\min }^{L}, a_{\min }^{U}\right]\right\},\left\{\left[b_{\max }^{L}, b_{\max }^{U}\right]\right\},\left\{\left[c_{\max }^{L}, c_{\max }^{U}\right]\right\}\right\}, \\
& \widehat{\wp}_{\max }=\left\{\widehat{\alpha}_{\max }, \widehat{\beta}_{\min }, \widehat{\gamma}_{\min }\right\}=\left\{\left\{\left[a_{\max }^{L}, a_{\max }^{U}\right]\right\},\left\{\left[b_{\min }^{L}, b_{\min }^{U}\right]\right\},\left\{\left[c_{\min }^{L}, c_{\min }^{U}\right]\right\}\right\}
\end{aligned}
$$

for

$$
\begin{aligned}
& a_{\min }^{L}=\bigcup_{\left[a_{\left(t_{k}\right)}^{L}, a_{\left(t_{k}\right)}^{U}\right] \in \widehat{\alpha}\left(t_{k}\right)} \min \left\{a_{\left(t_{k}\right)}^{L}\right\}, a_{\min }^{U}=\bigcup_{\left[a_{\left(t_{k}\right)}^{L}, a_{\left(t_{k}\right)}^{U}\right] \in \widehat{\alpha}\left(t_{k}\right)} \min \left\{a_{\left(t_{k}\right)}^{U}\right\}, \\
& a_{\max }^{L}=\bigcup_{\left[a_{\left(t_{k}\right)}^{L}, a_{\left(t_{k}\right)}^{U}\right] \in \widehat{\alpha}\left(t_{k}\right)} \max \left\{a_{\left(t_{k}\right)}^{L}\right\}, \\
& a_{\max }^{U}=\bigcup_{\left[a_{\left(t_{k}\right)}^{L}, a_{\left(t_{k}\right)}^{U}\right] \in \widehat{\alpha}\left(t_{k}\right)} \max \left\{a^{U}\right\}, b_{\min }^{L}=\bigcup_{\left[b_{\left(t_{k}\right)}^{L}, b_{\left(t_{k}\right)}^{U}\right] \in \widehat{\beta}\left(t_{k}\right)} \min \left\{b_{\left(t_{k}\right)}^{L}\right\}, \\
& b_{\min }^{U}=\bigcup_{\left[b_{\left(t_{k}\right)}^{L}, b_{\left(t_{k}\right)}^{U}\right] \in \widehat{\beta}\left(t_{k}\right)} \min \left\{b_{\left(t_{k}\right)}^{U}\right\}, \\
& b_{\max }^{L}=\bigcup_{\left[b_{\left(t_{k}\right)}^{L}, b_{\left(t_{k}\right)}^{U}\right] \in \widehat{\beta}\left(t_{k}\right)} \max \left\{b_{\left(t_{k}\right)}^{L}\right\}, b_{\max }^{U}=\bigcup_{\left[b_{\left(t_{k}\right)}^{L}, b_{\left(t_{k}\right)}^{U}\right] \in \widehat{\beta}\left(t_{k}\right)} \max \left\{b_{\left(t_{k}\right)}^{U}\right\} \text {, } \\
& c_{\min }^{L}=\bigcup_{\left[c_{\left(t_{k}\right)}^{L}, c_{\left(t_{k}\right)}^{U}\right] \in \widehat{\gamma}\left(t_{k}\right)} \min \left\{c_{\left(t_{k}\right)}^{L}\right\}, \\
& c_{\min }^{U}=\bigcup_{\left[c_{\left(t_{k}\right)}^{L}, c_{\left(t_{k}\right)}^{U}\right] \in \widehat{\gamma}\left(t_{k}\right)} \min \left\{c_{\left(t_{k}\right)}^{U}\right\}, c_{\max }^{L}=\bigcup_{\left[c_{\left(t_{k}\right)}^{L}, c_{\left(t_{k}\right)}^{U}\right] \in \widehat{\gamma}\left(t_{k}\right)} \max \left\{c_{\left(t_{k}\right)}^{L}\right\}, \\
& c_{\max }^{U}=\bigcup_{\left[c_{\left(t_{k}\right)}^{L}, c_{\left(t_{k}\right)}^{U}\right] \in \widehat{\gamma}\left(t_{k}\right)} \max \left\{c_{\left(t_{k}\right)}^{U}\right\} .
\end{aligned}
$$

Proof Let us prove (2), the others can be demonstrate similar to this.

Since $a_{\min }^{L} \leq a_{\left(t_{k}\right)}^{L} \leq a_{\max }^{L}$, then

$$
\begin{aligned}
1-a_{\left(t_{k}\right)}^{L} \leq 1 & -a_{\min }^{L} \Leftrightarrow 1-\left(1-a_{\left(t_{k}\right)}^{L}\right)^{\lambda} \geq 1-\left(1-a_{\min }^{L}\right)^{\lambda} \Leftrightarrow\left(1-\left(1-a_{\left(t_{k}\right)}^{L}\right)^{\lambda}\right)^{\omega\left(t_{k}\right)} \\
& \geq\left(1-\left(1-a_{\min }^{L}\right)^{\lambda}\right)^{\omega\left(t_{k}\right)} \\
& \Leftrightarrow \prod_{k=1}^{r}\left(1-\left(1-a_{\left(t_{k}\right)}^{L}\right)^{\lambda}\right)^{\omega\left(t_{k}\right)} \geq \prod_{k=1}^{r}\left(1-\left(1-a_{\min }^{L}\right)^{\lambda}\right)^{\omega\left(t_{k}\right)} \\
& \Leftrightarrow\left(1-\prod_{k=1}^{r}\left(1-\left(1-a_{\left(t_{k}\right)}^{L}\right)^{\lambda}\right)^{\omega\left(t_{k}\right)}\right)^{\frac{1}{\lambda}}
\end{aligned}
$$




$$
\begin{aligned}
& \leq\left(1-\prod_{k=1}^{r}\left(1-\left(1-a_{\min }^{L}\right)^{\lambda}\right)^{\omega\left(t_{k}\right)}\right)^{\frac{1}{\lambda}} \\
& \Leftrightarrow 1-\left(1-\prod_{k=1}^{r}\left(1-\left(1-a_{\left(t_{k}\right)}^{L}\right)^{\lambda}\right)^{\omega\left(t_{k}\right)}\right)^{\frac{1}{\lambda}} \\
& \geq 1-\left(1-\prod_{k=1}^{r}\left(1-\left(1-a_{\min }^{L}\right)^{\lambda}\right)^{\omega\left(t_{k}\right)}\right)^{\frac{1}{\lambda}}=a_{\min }^{L} .
\end{aligned}
$$

Similarly, we have

$$
1-\left(1-\prod_{k=1}^{r}\left(1-\left(1-a_{\left(t_{k}\right)}^{L}\right)^{\lambda}\right)^{\omega\left(t_{k}\right)}\right)^{\frac{1}{\lambda}} \leq 1-\left(1-\prod_{k=1}^{r}\left(1-\left(1-a_{\max }^{L}\right)^{\lambda}\right)^{\omega\left(t_{k}\right)}\right)^{\frac{1}{\lambda}}=a_{\max }^{L} .
$$

Likewise, since $a_{\min }^{U} \leq a_{\left(t_{k}\right)}^{U} \leq a_{\max }^{U}$, we obtain that

$$
1-\left(1-\prod_{k=1}^{r}\left(1-\left(1-a_{\left(t_{k}\right)}^{U}\right)^{\lambda}\right)^{\omega\left(t_{k}\right)}\right)^{\frac{1}{\lambda}} \geq 1-\left(1-\prod_{k=1}^{r}\left(1-\left(1-a_{\min }^{U}\right)^{\lambda}\right)^{\omega\left(t_{k}\right)}\right)^{\frac{1}{\lambda}}=a_{\min }^{U} .
$$

and

$$
1-\left(1-\prod_{k=1}^{r}\left(1-\left(1-a_{\left(t_{k}\right)}^{U}\right)^{\lambda}\right)^{\omega\left(t_{k}\right)}\right)^{\frac{1}{\lambda}} \leq 1-\left(1-\prod_{k=1}^{r}\left(1-\left(1-a_{\max }^{U}\right)^{\lambda}\right)^{\omega\left(t_{k}\right)}\right)^{\frac{1}{\lambda}}=a_{\max }^{U} .
$$

Considering $b_{\min }^{U} \leq b_{\left(t_{k}\right)}^{U} \leq b_{\max }^{U}$, we found

$$
\begin{aligned}
b_{\left(t_{k}\right)}^{U} \leq & b_{\max }^{U} \Leftrightarrow\left(b_{\left(t_{k}\right)}^{U}\right)^{\lambda} \leq\left(b_{\max }^{U}\right)^{\lambda} \Leftrightarrow 1-\left(b_{\left(t_{k}\right)}^{U}\right)^{\lambda} \\
\geq & 1-\left(b_{\max }^{U}\right)^{\lambda} \Leftrightarrow\left(1-\left(b_{\left(t_{k}\right)}^{U}\right)^{\lambda}\right)^{\omega\left(t_{k}\right)} \geq\left(1-\left(b_{\max }^{U}\right)^{\lambda}\right)^{\omega\left(t_{k}\right)} \\
\Leftrightarrow & \prod_{k=1}^{r}\left(1-\left(b_{\left(t_{k}\right)}^{U}\right)^{\lambda}\right)^{\omega\left(t_{k}\right)} \geq \prod_{k=1}^{r}\left(1-\left(b_{\max }^{U}\right)^{\lambda}\right)^{\omega\left(t_{k}\right)} \Leftrightarrow 1-\prod_{k=1}^{r}\left(1-\left(b_{\left(t_{k}\right)}^{U}\right)^{\lambda}\right)^{\omega\left(t_{k}\right)} \\
\leq & 1-\prod_{k=1}^{r}\left(1-\left(b_{\max }^{U}\right)^{\lambda}\right)^{\omega\left(t_{k}\right)} \\
& \times\left(1-\prod_{k=1}^{r}\left(1-\left(b_{\left(t_{k}\right)}^{U}\right)^{\lambda}\right)^{\omega\left(t_{k}\right)}\right)^{\frac{1}{\lambda}} \leq\left(1-\prod_{k=1}^{r}\left(1-\left(b_{\max }^{U}\right)^{\lambda}\right)^{\omega\left(t_{k}\right)}\right)^{\frac{1}{\lambda}}=b_{\max }^{U} .
\end{aligned}
$$

Similarly, we have

$$
\left(1-\prod_{k=1}^{r}\left(1-\left(b_{\left(t_{k}\right)}^{U}\right)^{\lambda}\right)^{\omega\left(t_{k}\right)}\right)^{\frac{1}{\lambda}} \geq\left(1-\prod_{k=1}^{r}\left(1-\left(b_{\min }^{U}\right)^{\lambda}\right)^{\omega\left(t_{k}\right)}\right)^{\frac{1}{\lambda}}=b_{\min }^{U} .
$$

Likewise, since $b_{\min }^{L} \leq b_{\left(t_{k}\right)}^{L} \leq b_{\max }^{L}$, we obtain that

$$
b_{\min }^{L}=\left(1-\prod_{k=1}^{r}\left(1-\left(b_{\min }^{L}\right)^{\lambda}\right)^{\omega\left(t_{k}\right)}\right)^{\frac{1}{\lambda}} \leq\left(1-\prod_{k=1}^{r}\left(1-\left(b_{\left(t_{k}\right)}^{L}\right)^{\lambda}\right)^{\omega\left(t_{k}\right)}\right)^{\frac{1}{\lambda}}
$$




$$
\leq\left(1-\prod_{k=1}^{r}\left(1-\left(b_{\max }^{L}\right)^{\lambda}\right)^{\omega\left(t_{k}\right)}\right)^{\frac{1}{\lambda}}=b_{\max }^{L}
$$

For $c_{\min }^{U} \leq c_{\left(t_{k}\right)}^{U} \leq c_{\max }^{U}$ and $c_{\min }^{L} \leq c_{\left(t_{k}\right)}^{L} \leq c_{\max }^{L}$, we have

$c_{\min }^{L} \leq\left(1-\prod_{k=1}^{r}\left(1-\left(c_{\left(t_{k}\right)}^{L}\right)^{\lambda}\right)^{\omega\left(t_{k}\right)}\right)^{\frac{1}{\lambda}} \leq c_{\max }^{L}$, and $c_{\min }^{U} \leq\left(1-\prod_{k=1}^{r}\left(1-\left(c_{\left(t_{k}\right)}^{U}\right)^{\lambda}\right)^{\omega\left(t_{k}\right)}\right)^{\frac{1}{\lambda}} \leq c_{\max }^{U}$.

Let $G I V P H F W G_{\omega(t)}\left(\widehat{\wp}\left(t_{1}\right), \widehat{\wp}\left(t_{2}\right), \ldots, \widehat{\wp}\left(t_{r}\right)\right)=\widehat{\wp}=\{\widehat{\alpha}, \widehat{\beta}, \widehat{\gamma}\}$ (from Theorem 3.6), then we say that

$$
\begin{aligned}
& \mathcal{S}(\widehat{\wp})=\frac{1+\frac{1}{2}\left(\frac{1}{v(\alpha)} \sum_{\left[a^{L}, a^{U}\right] \in \widehat{\alpha}}\left(a^{L}+a^{U}\right)-\frac{1}{v(\hat{\beta})} \sum_{\left[b^{L}, b^{U}\right] \in \hat{\beta}}\left(b^{L}+b^{U}\right)-\frac{1}{v(\hat{\gamma})} \sum_{\left[c^{L}, c^{U}\right] \widehat{\gamma} \hat{\gamma}}\left(c^{L}+c^{U}\right)\right)}{2}
\end{aligned}
$$

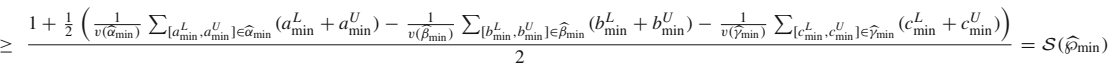

$$
\begin{aligned}
& \mathcal{S}(\widehat{\wp})=\frac{1+\frac{1}{2}\left(\frac{1}{v(\alpha)} \sum_{\left[a^{L}, a^{U}\right] \in \widehat{\alpha}}\left(a^{L}+a^{U}\right)-\frac{1}{v(\hat{\beta})} \sum_{\left[b^{L}, b^{U}\right] \in \widehat{\beta}}\left(b^{L}+b^{U}\right)-\frac{1}{v(\hat{\gamma})} \sum_{\left[c^{L}, c^{U}\right] \in \hat{\gamma}}\left(c^{L}+c^{U}\right)\right)}{2}
\end{aligned}
$$

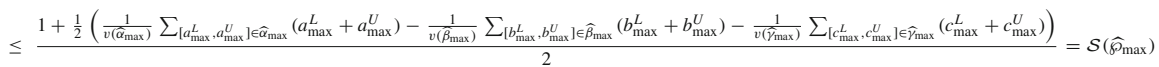

Then, we obtain $\widehat{\wp}_{\min } \leq G D I V P H F W G_{\omega(t)}\left(\widehat{\wp}\left(t_{1}\right), \widehat{\wp}\left(t_{2}\right), \ldots, \widehat{\wp}\left(t_{r}\right)\right) \leq \widehat{\wp}_{\max }$. Thus, the boundary property is verified.

Theorem 3.9 (Monotonicity) Let $\left(\widehat{\wp}\left(t_{1}\right), \widehat{\wp}\left(t_{2}\right), \ldots, \widehat{\wp}\left(t_{r}\right)\right)$ and $\left(\widehat{\wp}^{*}\left(t_{1}\right), \widehat{\wp}^{*}\left(t_{2}\right), \ldots, \widehat{\wp}^{*}\left(t_{r}\right)\right)$ be two collections of the IVPHFEs collected from $k$ different periods $t_{k}(k=1,2, \ldots, r)$. If $\widehat{\wp}\left(t_{k}\right) \leq \widehat{\wp}^{*}\left(t_{k}\right)$ for all $k=1,2, \ldots, r$ then

1. GDIVPHFW $A_{\omega(t)}\left(\widehat{\wp}\left(t_{1}\right), \widehat{\wp}\left(t_{2}\right), \ldots, \widehat{\wp}\left(t_{r}\right)\right) \leq G D I V P H F W A_{\omega(t)}\left(\widehat{\wp}^{*}\left(t_{1}\right), \widehat{\wp}^{*}\left(t_{2}\right)\right.$, $\left.\ldots, \widehat{\wp}^{*}\left(t_{r}\right)\right)$.

2. GDIVPHFW $G_{\omega(t)}\left(\widehat{\wp}\left(t_{1}\right), \widehat{\wp}\left(t_{2}\right), \ldots, \widehat{\wp}\left(t_{r}\right)\right) \leq G D I V P H F W G_{\omega(t)}\left(\widehat{\wp}^{*}\left(t_{1}\right), \widehat{\wp}^{*}\left(t_{2}\right)\right.$, ..., $\left.\widehat{\wp}^{*}\left(t_{r}\right)\right)$.

Proof By considering Eqs. (3.11) and (3.12), they can be interpreted similar to the proof of Theorem 4.8.

Theorem 3.10 (Commutativity) Let $\left(\widehat{\wp}\left(t_{1}\right), \widehat{\wp}\left(t_{2}\right), \ldots, \widehat{\wp}\left(t_{r}\right)\right)$ be a collections of IVPHFEs collected from $k$ different periods $t_{k}(k=1,2, \ldots, r)$. In addition, let $\widehat{\wp_{n}}\left(t_{1_{n}}\right), \widehat{\wp_{n}}\left(t_{2_{n}}\right)$, $\ldots, \widehat{\wp n}\left(t_{r_{n}}\right)$ be a new permutation of $\widehat{\wp}\left(t_{1}\right), \widehat{\wp}\left(t_{2}\right), \ldots, \widehat{\wp}\left(t_{r}\right)$ then

1. GDIVPHFWA $A_{\omega(t)}\left(\widehat{\wp}\left(t_{1}\right), \widehat{\wp}\left(t_{2}\right), \ldots, \widehat{\wp}\left(t_{r}\right)\right)=G D I V P H F W A_{\omega(t)}\left(\widehat{\wp_{n}}\left(t_{1_{n}}\right), \widehat{\wp_{n}}\left(t_{2_{n}}\right)\right.$, .., $\left.\widehat{\rho_{n}}\left(t_{r_{n}}\right)\right)$.

2. $G D I V P H F W G_{\omega(t)}\left(\widehat{\wp}\left(t_{1}\right), \widehat{\wp}\left(t_{2}\right), \ldots, \widehat{\wp}\left(t_{r}\right)\right)=G D I V P H F W G_{\omega(t)}\left(\widehat{\wp n}\left(t_{1_{n}}\right), \widehat{\wp_{n}}\left(t_{2_{n}}\right)\right.$, $\left.\ldots, \widehat{\wp_{n}}\left(t_{r_{n}}\right)\right)$.

Proof It can be easily seen by Theorem 3.6 (3) and (4).

\section{Dynamic interval-valued picture hesitant fuzzy weighted aggregation operators based on Einstein operations}

In this part, we derive the dynamic interval-valued picture hesitant fuzzy weighted aggregation operators based on Einstein operations to fuse interval-valued picture hesitant fuzzy tools acquired at different time-moments. 
Einstein operations include the Einstein sum and Einstein products (Klement et al. 2004). Einstein sum $\oplus_{\epsilon}$ is a t-conorm and Einstein product $\otimes_{\epsilon}$ is a t-norm, where for all $(x, y) \epsilon$ $[0,1] \times[0,1]$

$$
x \oplus_{\epsilon} y=\frac{x+y}{1+x+y} \quad \text { and } \quad x \otimes_{\epsilon} y=\frac{x+y}{1+(1-x)(1-y)} .
$$

Definition 4.1 Let $\widehat{\wp}\left(t_{1}\right)=\left\{\widehat{\alpha}\left(t_{1}\right), \widehat{\beta}\left(t_{1}\right), \widehat{\gamma}\left(t_{1}\right)\right\}$ and $\widehat{\wp}\left(t_{2}\right)=\left\{\widehat{\alpha}\left(t_{2}\right), \widehat{\beta}\left(t_{2}\right), \widehat{\gamma}\left(t_{2}\right)\right\}$ be two IVPHFEs and $\sigma$ be any positive real number (i.e., $\sigma>0$ ). Then, the Einstein operations of IVPHFEs are described as

(a)

$$
\begin{aligned}
& \widehat{\wp}\left(t_{1}\right) \oplus_{\epsilon} \widehat{\wp}\left(t_{2}\right)
\end{aligned}
$$

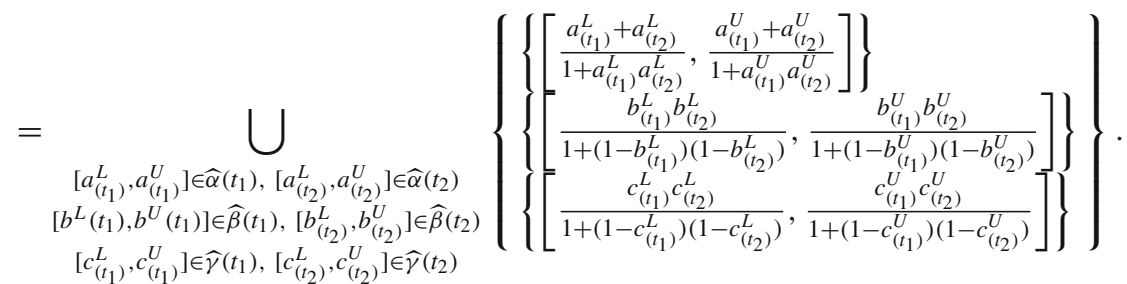

(b)

$\widehat{\wp}\left(t_{1}\right) \otimes_{\epsilon} \widehat{\wp}\left(t_{2}\right)$

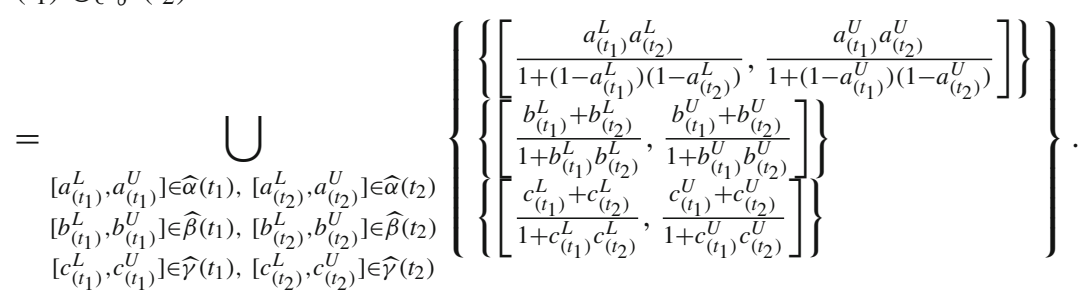

(c)

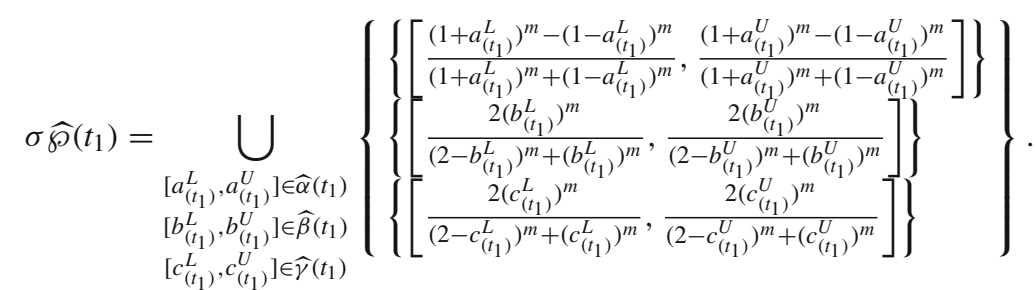

(d)

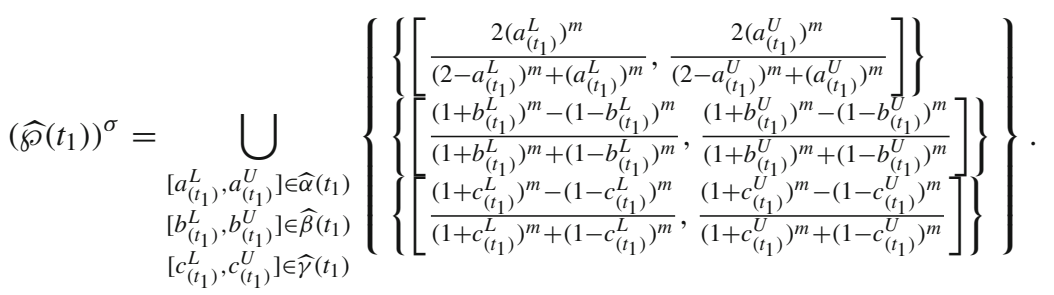


Example 4.2 We consider $\widehat{\wp}\left(t_{1}\right)$ and $\widehat{\wp}\left(t_{2}\right)$ given in Example 3.3. Also let $\sigma=2$. Then, we obtain

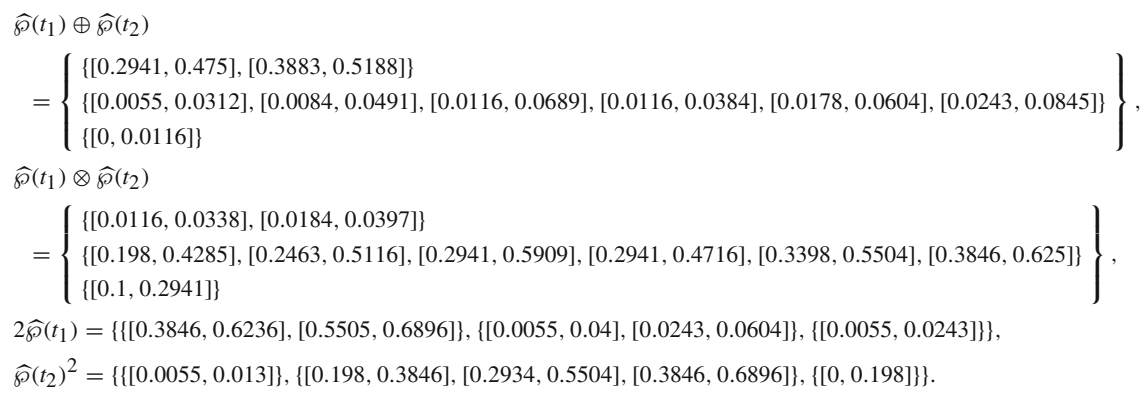

Proposition 4.3 Let $\widehat{\wp}_{1}=\left\{\widehat{\alpha}_{1}, \widehat{\beta}_{1}, \widehat{\gamma}_{1}\right\}$ and $\widehat{\wp}_{2}=\left\{\widehat{\alpha}_{2}, \widehat{\beta}_{2}, \widehat{\gamma}_{2}\right\}$ be two IVPHFEs and $\sigma>0$.

1. $\widehat{\varnothing}\left(t_{1}\right) \oplus_{\epsilon} \widehat{\wp}\left(t_{2}\right)=\widehat{\wp}\left(t_{2}\right) \oplus_{\epsilon} \widehat{\varnothing}\left(t_{1}\right)$.

2. $\widehat{\wp}\left(t_{1}\right) \otimes_{\epsilon} \widehat{\wp}\left(t_{2}\right)=\widehat{\wp}\left(t_{2}\right) \otimes_{\epsilon} \widehat{\wp}\left(t_{1}\right)$.

3. $\sigma\left(\widehat{\wp}\left(t_{1}\right) \oplus_{\epsilon} \widehat{\wp}\left(t_{2}\right)\right)=\sigma \widehat{\wp}\left(t_{1}\right) \oplus_{\epsilon} \sigma \widehat{\wp}\left(t_{2}\right)$.

4. $\left(\widehat{\wp}\left(t_{1}\right) \otimes_{\epsilon} \widehat{\wp}\left(t_{2}\right)\right)^{\sigma}=\left(\widehat{\wp}\left(t_{1}\right)\right)^{\sigma} \otimes_{\epsilon}\left(\widehat{\wp}\left(t_{2}\right)\right)^{\sigma}$.

Proof It is trivial from Definition 4.1.

Considering the above Einstein operation laws of IVPHFEs, we develop the following Einstein weighted aggregation operators.

Definition 4.4 Let $\widehat{\wp}\left(t_{k}\right)(k=1,2, \ldots, r)$ be the IVPHFEs collected from $k$ different periods $t_{k}(k=1,2, \ldots, r)$. In addition, let $\omega(t)=\left(\omega\left(t_{1}\right), \omega\left(t_{2}\right), \ldots, \omega\left(t_{r}\right)\right)^{T}$ be the weighted vector of $t_{k}(k=1,2, \ldots, r)$, with $\omega\left(t_{k}\right) \in[0,1]$ and $\sum_{k=1}^{r} \omega\left(t_{k}\right)=1$, and $\lambda>0$.

(a) A dynamic interval-valued picture hesitant fuzzy Einstein weighted average (DIVPHFEWA) operator is a mapping DIVPHFEW $A_{\epsilon}^{\omega(t)}: \mathcal{P}^{r} \rightarrow \mathcal{P}$, such that

$$
\begin{aligned}
& D I V P H F E W A_{\epsilon}^{\omega(t)}\left(\widehat{\wp}\left(t_{1}\right), \widehat{\wp}\left(t_{2}\right), \ldots, \widehat{\wp}\left(t_{r}\right)\right) \\
& =\bigoplus_{k=1}^{r} \omega\left(t_{k}\right) \widehat{\wp}\left(t_{k}\right)=\omega\left(t_{1}\right) \widehat{\wp}\left(t_{1}\right) \oplus_{\epsilon} \omega\left(t_{2}\right) \widehat{\wp}\left(t_{2}\right) \oplus_{\epsilon} \ldots \oplus_{\epsilon} \omega\left(t_{r}\right) \widehat{\wp}\left(t_{r}\right)
\end{aligned}
$$

(b) A dynamic interval-valued picture hesitant fuzzy Einstein weighted geometric (DIVPHFEWG) operator is a mapping DIVPHFEWG $G_{\epsilon}^{(t)}: \mathcal{P}^{r} \rightarrow \mathcal{P}$, such that

$$
\begin{aligned}
& D I V P H F E W G_{\epsilon}^{\omega(t)}\left(\widehat{\wp}\left(t_{1}\right), \widehat{\wp}\left(t_{2}\right), \ldots, \widehat{\wp}\left(t_{r}\right)\right) \\
& =\bigotimes_{k=1}^{r}\left(\widehat{\wp}\left(t_{k}\right)\right)^{\omega\left(t_{k}\right)}=\left(\widehat{\wp}\left(t_{1}\right)\right)^{\omega\left(t_{1}\right)} \otimes_{\epsilon}\left(\widehat{\wp}\left(t_{2}\right)\right)^{\omega\left(t_{2}\right)} \otimes_{\epsilon} \ldots \otimes_{\epsilon}\left(\widehat{\wp}\left(t_{r}\right)\right)^{\omega\left(t_{r}\right)}
\end{aligned}
$$

(c) A generalized dynamic interval-valued picture hesitant fuzzy Einstein weighted average (GDIVPHFEWA) operator is a mapping GDIVPHFEW $A_{\epsilon}^{\omega(t)}: \mathcal{P}^{r} \rightarrow \mathcal{P}$, such that

$$
\begin{aligned}
& \text { GDIV P H F EW } A_{\epsilon}^{\omega(t)}\left(\widehat{\wp}\left(t_{1}\right), \widehat{\wp}\left(t_{2}\right), \ldots, \widehat{\wp}\left(t_{r}\right)\right) \\
& =\left(\bigoplus_{k=1}^{r} \omega\left(t_{k}\right)\left(\widehat{\wp}\left(t_{k}\right)\right)^{\lambda}\right)^{\frac{1}{\lambda}}=\left(\omega\left(t_{1}\right)\left(\widehat{\wp}\left(t_{1}\right)\right)^{\lambda} \oplus_{\epsilon} \omega\left(t_{2}\right)\left(\widehat{\wp}\left(t_{2}\right)\right)^{\lambda} \oplus_{\epsilon} \ldots \oplus_{\epsilon} \omega\left(t_{k}\right)\left(\widehat{\wp}\left(t_{k}\right)\right)^{\lambda}\right)^{\frac{1}{\lambda}}
\end{aligned}
$$


(d) A generalized dynamic interval-valued picture hesitant fuzzy Einstein weighted geometric (GDIVPHFEWG) operator is a mapping GDIVPHFEWG $G_{\epsilon}^{\omega(t)}: \mathcal{P}^{r} \rightarrow \mathcal{P}$, such that

$$
\begin{aligned}
& \text { GDIV P H FEWG } G_{\epsilon}^{\omega(t)}\left(\widehat{\wp}\left(t_{1}\right), \widehat{\wp}\left(t_{2}\right), \ldots, \widehat{\wp}\left(t_{r}\right)\right) \\
& =\frac{1}{\lambda}\left(\bigotimes_{k=1}^{r} \lambda\left(\widehat{\wp}\left(t_{k}\right)\right)^{\omega\left(t_{k}\right)}\right)=\frac{1}{\lambda}\left(\lambda\left(\widehat{\wp}\left(t_{1}\right)\right)^{\omega\left(t_{1}\right)} \otimes_{\epsilon} \lambda\left(\widehat{\wp}\left(t_{2}\right)\right)^{\omega\left(t_{2}\right)} \otimes_{\epsilon} \ldots \otimes_{\epsilon} \lambda\left(\widehat{\wp}\left(t_{r}\right)\right)^{\omega\left(t_{r}\right)}\right)
\end{aligned}
$$

Theorem 4.5 Let $\widehat{\wp}\left(t_{k}\right)(k=1,2, \ldots, r)$ be the IVPHFEs collected from $k$ different periods $t_{k}(k=1,2, \ldots, r)$. Also, let $\omega(t)=\left(\omega\left(t_{1}\right), \omega\left(t_{2}\right), \ldots, \omega\left(t_{r}\right)\right)^{T}$ be the weighted vector of $t_{k}$ $(k=1,2, \ldots, r)$, with $\omega\left(t_{k}\right) \in[0,1]$ and $\sum_{k=1}^{r} \omega\left(t_{k}\right)=1$, and $\lambda>0$.

(1) The aggregation value of IVPHFEs by using the DIVPHFEWA operator is also an IVPHFE, and

$$
\begin{aligned}
& D I V P H F E W A_{\epsilon}^{\omega(t)}\left(\widehat{\wp}\left(t_{1}\right), \widehat{\wp}\left(t_{2}\right), \ldots, \widehat{\wp}\left(t_{r}\right)\right) \\
& =\bigoplus_{k=1}^{r} \omega\left(t_{k}\right) \widehat{\wp}\left(t_{k}\right)
\end{aligned}
$$

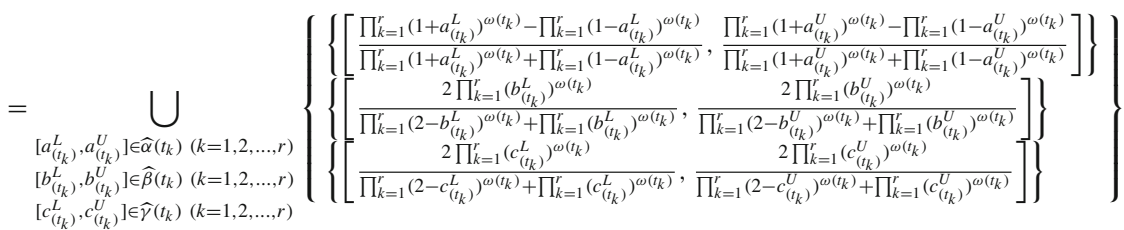

(2) The aggregation value of IVPHFEs by using the DIVPHFEWG operator is also an IVPHFE, and

$$
\begin{aligned}
& D I V P H F E W G_{\epsilon}^{\omega(t)}\left(\widehat{\wp}\left(t_{1}\right), \widehat{\wp}\left(t_{2}\right), \ldots, \widehat{\wp}\left(t_{r}\right)\right) \\
& =\bigotimes_{k=1}^{r}\left(\widehat{\wp}\left(t_{k}\right)\right)^{\omega\left(t_{k}\right)}
\end{aligned}
$$

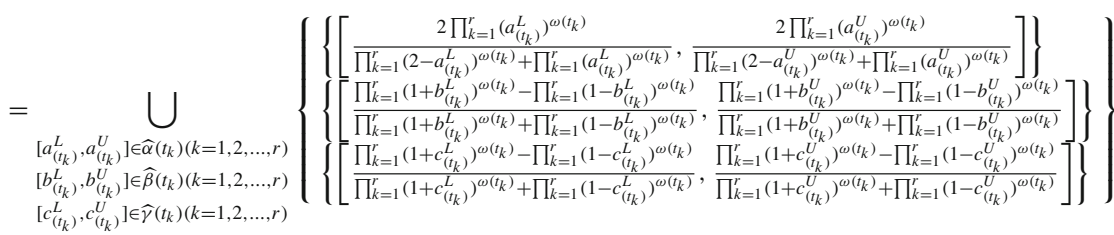

(3) The aggregation value of IVPHFEs by using the GDIVPHFEWA operator is also an IVPHFE, and

$$
\begin{aligned}
& \text { GDIVP P FEW } A_{\epsilon}^{\omega(t)}\left(\widehat{\wp}\left(t_{1}\right), \widehat{\wp}\left(t_{2}\right), \ldots, \widehat{\wp}\left(t_{r}\right)\right) \\
& =\left(\bigoplus_{k=1}^{r} \omega\left(t_{k}\right)\left(\widehat{\wp}\left(t_{k}\right)\right)^{\lambda}\right)^{\frac{1}{\lambda}}
\end{aligned}
$$




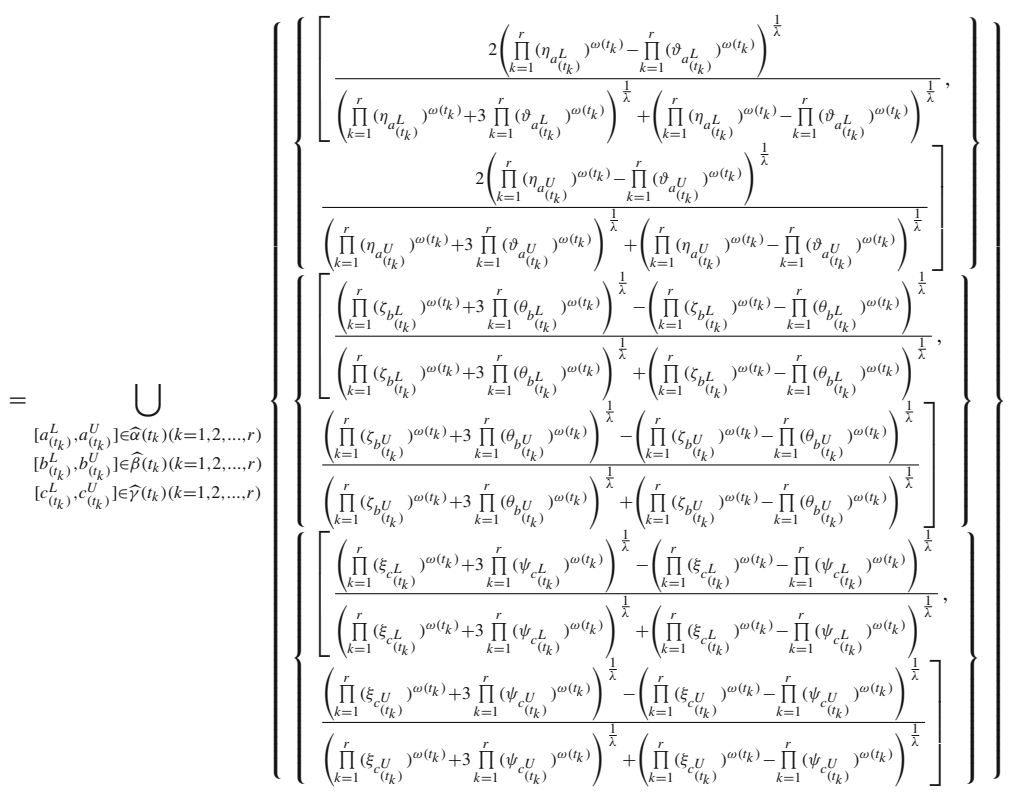

where

$$
\left\{\begin{array}{l}
\eta_{a_{\left(t_{k}\right)}^{L}}=\left(2-a_{\left(t_{k}\right)}^{L}\right)^{\lambda}+3\left(a_{\left(t_{k}\right)}^{L}\right)^{\lambda}, \vartheta_{a_{\left(t_{k}\right)}^{L}}^{L}=\left(2-a_{\left(t_{k}\right)}^{L}\right)^{\lambda}-\left(a_{\left(t_{k}\right)}^{L}\right)^{\lambda}, \\
\eta_{a_{\left(t_{k}\right)}^{U}}=\left(2-a_{\left(t_{k}\right)}^{U}\right)^{\lambda}+3\left(a_{\left(t_{k}\right)}^{U}\right)^{\lambda}, \vartheta_{a_{\left(t_{k}\right)}^{U}}^{U}=\left(2-a_{\left(t_{k}\right)}^{U}\right)^{\lambda}-\left(a_{\left(t_{k}\right)}^{U}\right)^{\lambda}, \\
\zeta_{b_{\left(t_{k}\right)}^{L}}^{L}=\left(1+b_{\left(t_{k}\right)}^{L}\right)^{\lambda}+3\left(1-b_{\left(t_{k}\right)}^{L}\right)^{\lambda}, \theta_{b_{\left(t_{k}\right)}^{L}}^{L}=\left(1+b_{\left(t_{k}\right)}^{L}\right)^{\lambda}-\left(1-b_{\left(t_{k}\right)}^{L}\right)^{\lambda}, \\
\zeta_{b_{\left(t_{k}\right)}^{U}}=\left(1+b_{\left(t_{k}\right)}^{U}\right)^{\lambda}+3\left(1-b_{\left(t_{k}\right)}^{U}\right)^{\lambda}, \theta_{b_{\left(t_{k}\right)}^{U}}^{U}=\left(1+b_{\left(t_{k}\right)}^{U}\right)^{\lambda}-\left(1-b_{\left(t_{k}\right)}^{U}\right)^{\lambda}, \\
\xi_{c_{\left(t_{k}\right)}^{L}}=\left(1+c_{\left(t_{k}\right)}^{L}\right)^{\lambda}+3\left(1-c_{\left(t_{k}\right)}^{L}\right)^{\lambda}, \psi_{c_{\left(t_{k}\right)}^{L}}^{L}=\left(1+c_{\left(t_{k}\right)}^{L}\right)^{\lambda}-\left(1-c_{\left(t_{k}\right)}^{L}\right)^{\lambda}, \\
\xi_{c_{\left(t_{k}\right)}^{U}}^{L}=\left(1+c_{\left(t_{k}\right)}^{U}\right)^{\lambda}+3\left(1-c_{\left(t_{k}\right)}^{U}\right)^{\lambda}, \psi_{c_{\left(t_{k}\right)}^{U}}^{U}=\left(1+c_{\left(t_{k}\right)}^{U}\right)^{\lambda}-\left(1-c_{\left(t_{k}\right)}^{U}\right)^{\lambda} .
\end{array}\right.
$$

4. The aggregation value of IVPHFEs by using the GDIVPHFEWG operator is also an IVPHFE, and

GDIVP H FEW $G_{\epsilon}^{\omega(t)}\left(\widehat{\wp}\left(t_{1}\right), \widehat{\wp}\left(t_{2}\right), \ldots, \widehat{\wp}\left(t_{r}\right)\right)$

$$
=\frac{1}{\lambda}\left(\bigotimes_{k=1}^{r} \lambda\left(\widehat{\wp}\left(t_{k}\right)\right)^{\omega\left(t_{k}\right)}\right)
$$




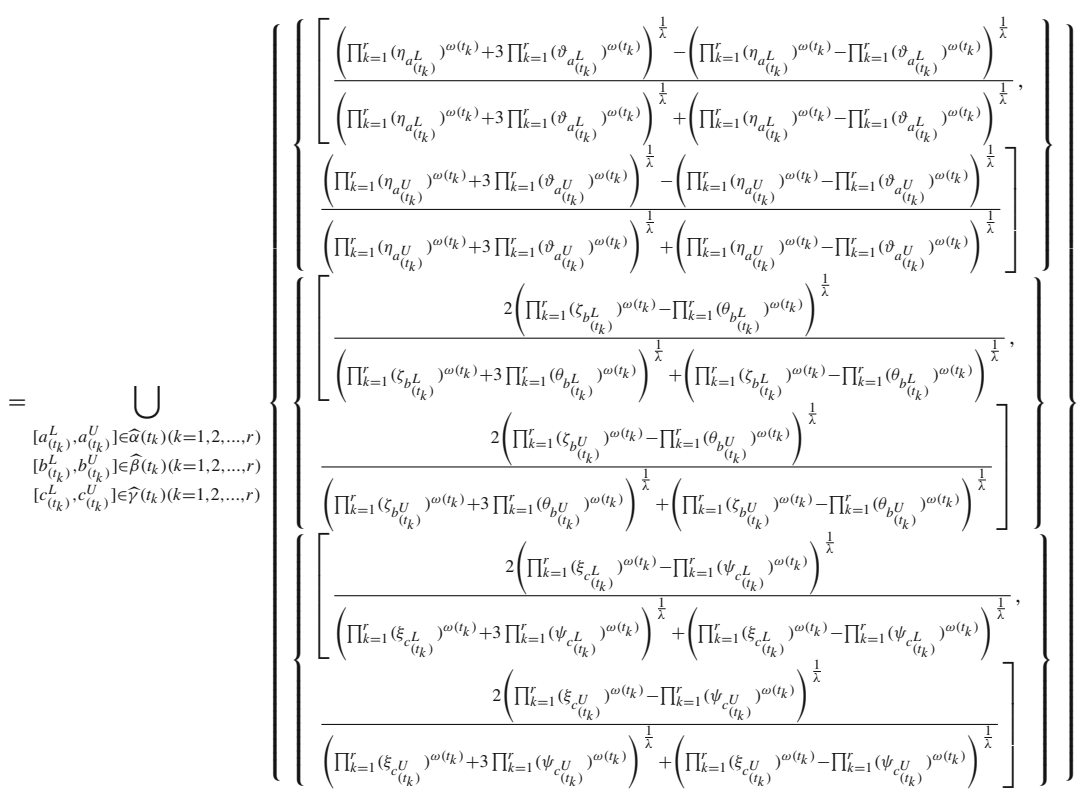

where

$$
\left\{\begin{array}{l}
\eta_{a_{\left(t_{k}\right)}^{L}}=\left(1+a_{\left(t_{k}\right)}^{L}\right)^{\lambda}+3\left(1-a_{\left(t_{k}\right)}^{L}\right)^{\lambda}, \quad \vartheta_{a_{\left(t_{k}\right)}^{L}}=\left(1+a_{\left(t_{k}\right)}^{L}\right)^{\lambda}-\left(1-a_{\left(t_{k}\right)}^{L}\right)^{\lambda}, \\
\eta_{a_{\left(t_{k}\right)}^{U}}=\left(1+a_{\left(t_{k}\right)}^{U}\right)^{\lambda}+3\left(1-a_{\left(t_{k}\right)}^{U}\right)^{\lambda}, \quad \vartheta_{a_{\left(t_{k}\right)}^{U}}=\left(1+a_{\left(t_{k}\right)}^{U}\right)^{\lambda}-\left(1-a_{\left(t_{k}\right)}^{U}\right)^{\lambda}, \\
\zeta_{b_{\left(t_{k}\right)}^{L}}=\left(2-b_{\left(t_{k}\right)}^{L}\right)^{\lambda}+3\left(b_{\left(t_{k}\right)}^{L}\right)^{\lambda}, \theta_{b_{\left(t_{k}\right)}^{L}}=\left(2-b_{\left(t_{k}\right)}^{L}\right)^{\lambda}-\left(b_{\left(t_{k}\right)}^{L}\right)^{\lambda}, \\
\zeta_{b_{\left(t_{k}\right)}^{U}}=\left(2-b_{\left(t_{k}\right)}^{U}\right)^{\lambda}+3\left(b_{\left(t_{k}\right)}^{U}\right)^{\lambda}, \theta_{b_{\left(t_{k}\right)}^{U}}=\left(2-b_{\left(t_{k}\right)}^{U}\right)^{\lambda}-\left(b_{\left(t_{k}\right)}^{U}\right)^{\lambda}, \\
\xi_{c_{\left(t_{k}\right)}^{L}}^{L}=\left(2-c_{\left(t_{k}\right)}^{L}\right)^{\lambda}+3\left(c_{\left(t_{k}\right)}^{L}\right)^{\lambda}, \quad \psi_{c_{\left(t_{k}\right)}^{L}}^{L}=\left(2-c_{\left(t_{k}\right)}^{L}\right)^{\lambda}-\left(c_{\left(t_{k}\right)}^{L}\right)^{\lambda}, \\
\xi_{c_{\left(t_{k}\right)}^{U}}^{U}=\left(2-c_{\left(t_{k}\right)}^{U}\right)^{\lambda}+3\left(c_{\left(t_{k}\right)}^{U}\right)^{\lambda}, \quad \psi_{c_{\left(t_{k}\right)}^{U}}^{U}=\left(2-c_{\left(t_{k}\right)}^{U}\right)^{\lambda}-\left(c_{\left(t_{k}\right)}^{U}\right)^{\lambda} .
\end{array}\right.
$$

Proof Let us prove (3), the others can be demonstrate similar to this.

We use the induction technique for proof.

- For $r=1$ :

Then we obtain the following

$$
G D I V P H F E W A_{\epsilon}^{\omega(t)}\left(\widehat{\wp}\left(t_{1}\right)\right)=\left(\omega\left(t_{1}\right)\left(\widehat{\wp}\left(t_{1}\right)\right)^{\lambda}\right)^{\frac{1}{\lambda}}=\widehat{\wp}\left(t_{1}\right) .
$$

Because from Definition 4.1 (d) we calculate $\left(\widehat{\wp}\left(t_{1}\right)\right)^{\lambda}$ as

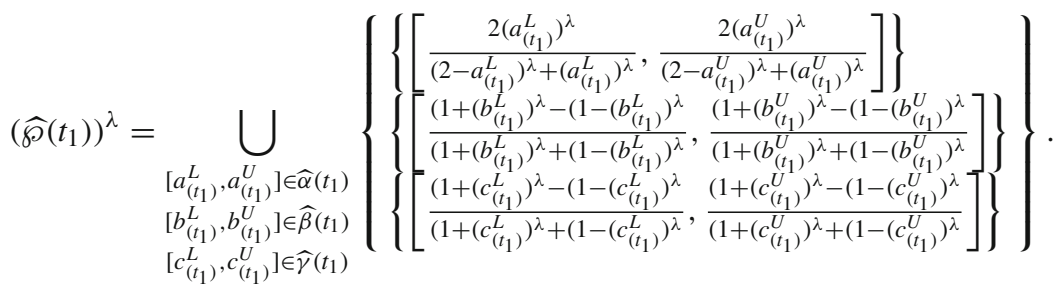


Since $\omega\left(t_{1}\right)=1$, we have $\omega\left(t_{1}\right)\left(\widehat{\wp}\left(t_{1}\right)\right)^{\lambda}=\left(\widehat{\wp}\left(t_{1}\right)\right)^{\lambda}$ by Definition 4.1 (c). Then, we obtain that (from Definition 4.1 (d))

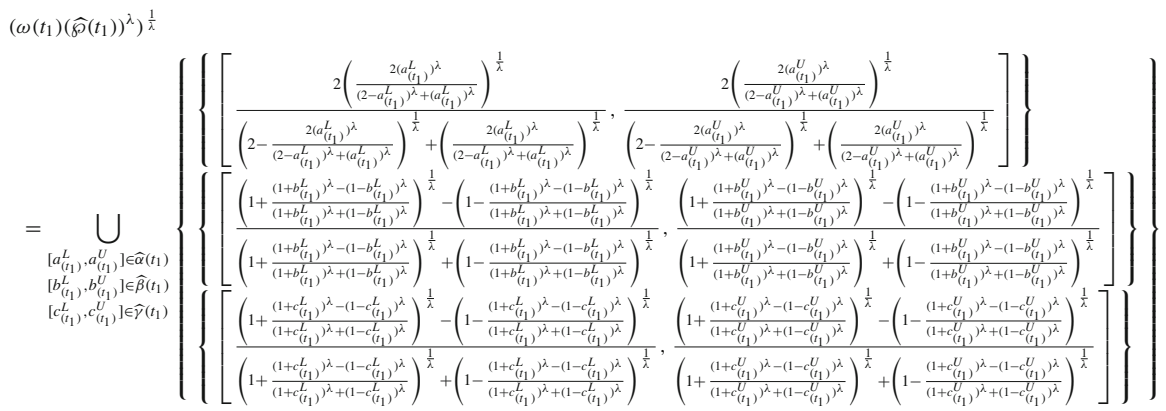

We demonstrate that

$$
\begin{gathered}
\frac{2\left(\frac{2\left(a_{\left(t_{1}\right)}^{L}\right)^{\lambda}}{\left(2-a_{\left(t_{1}\right)}^{L}\right)^{\lambda}+\left(a_{\left(t_{1}\right)}^{L}\right)^{\lambda}}\right)^{\frac{1}{\lambda}}}{\left(2-\frac{2\left(a_{\left(t_{1}\right)}^{L}\right)^{\lambda}}{\left(2-a_{\left(t_{1}\right)}^{L}\right)^{\lambda}+\left(a_{\left(t_{1}\right)}^{L}\right)^{\lambda}}\right)^{\frac{1}{\lambda}}+\left(\frac{2\left(a_{\left(t_{1}\right)}^{L}\right)^{\lambda}}{\left(2-a_{\left(t_{1}\right)}^{L}\right)^{\lambda}+\left(a_{\left(t_{1}\right)}^{L}\right)^{\lambda}}\right)^{\frac{1}{\lambda}}}=\frac{\frac{2^{\frac{\lambda+1}{\lambda}}\left(\left(a_{\left(t_{1}\right.}^{L}\right)^{\lambda}\right)^{\frac{1}{\lambda}}}{\left(\left(2-a_{\left(t_{1}\right)}^{L}\right)^{\lambda}+\left(a_{\left(t_{1}\right)}^{L}\right)^{\lambda}\right)^{\frac{1}{\lambda}}}}{\frac{2^{\frac{1}{\lambda}}\left(\left(2-a_{\left(t_{1}\right)}^{L}\right)^{\lambda}\right)^{\frac{1}{\lambda}}+\left(\left(a_{\left(t_{1}\right.}^{L}\right)^{\lambda}\right)^{\frac{1}{\lambda}}}{\left(\left(2-a_{\left(t_{1}\right)}^{L}\right)^{\lambda}+\left(a_{\left(t_{1}\right)}^{L}\right)^{\lambda}\right)^{\frac{1}{\lambda}}}} \\
=\frac{2 a_{\left(t_{1}\right)}^{L}=a_{\left(t_{1}\right)}^{L}}{2}
\end{gathered}
$$

and

$$
\begin{aligned}
& \frac{\left(1+\frac{\left(1+b_{\left(t_{1}\right)}^{L}\right)^{\lambda}-\left(1-b_{\left(t_{1}\right)}^{L}\right)^{\lambda}}{\left(1+b_{\left(t_{1}\right)}^{L}\right)^{\lambda}+\left(1-b_{\left(t_{1}\right)}^{L}\right)^{\lambda}}\right)^{\frac{1}{\lambda}}-\left(1-\frac{\left(1+b_{\left(t_{1}\right)}^{L}\right)^{\lambda}-\left(1-b_{\left(t_{1}\right)}^{L}\right)^{\lambda}}{\left(1+b_{\left(t_{1}\right)}^{L}\right)^{\lambda}+\left(1-b_{\left(t_{1}\right)}^{L}\right)^{\lambda}}\right)^{\frac{1}{\lambda}}}{\left(1+\frac{\left(1+b_{\left(t_{1}\right)}^{L}\right)^{\lambda}-\left(1-b_{\left(t_{1}\right)}^{L}\right)^{\lambda}}{\left(1+b_{\left(t_{1}\right)}^{L}\right)^{\lambda}+\left(1-b_{\left(t_{1}\right)}^{L}\right)^{\lambda}}\right)^{\frac{1}{\lambda}}+\left(1-\frac{\left(1+b_{\left(t_{1}\right)}^{L}\right)^{\lambda}-\left(1-b_{\left(t_{1}\right)}^{L}\right)^{\lambda}}{\left(1+b_{\left(t_{1}\right)}^{L}\right)^{\lambda}+\left(1-b_{\left(t_{1}\right)}^{L}\right)^{\lambda}}\right)^{\frac{1}{\lambda}}} \\
& =\frac{\left(2\left(1+b_{\left(t_{1}\right)}^{L}\right)^{\lambda}\right)^{\frac{1}{\lambda}}-\left(2\left(1-b_{\left(t_{1}\right)}^{L}\right)^{\lambda}\right)^{\frac{1}{\lambda}}}{\left(\left(1+b_{\left(t_{1}\right)}^{L}\right)^{\lambda}-\left(1-b_{\left(t_{1}\right)}^{L}\right)^{\lambda}\right)^{\frac{1}{\lambda}}}=\frac{2 b_{\left(t_{1}\right)}^{L}}{2}=b_{\left(t_{1}\right)}^{L} \\
& \frac{\left(2\left(1+b_{\left(t_{1}\right)}^{L}\right)^{\lambda}\right)^{\frac{1}{\lambda}}-\left(2\left(1-b_{\left(t_{1}\right)}^{L}\right)^{\lambda}\right)^{\frac{1}{\lambda}}}{\left(\left(1+b_{\left(t_{1}\right)}^{L}\right)^{\lambda}-\left(1-b_{\left(t_{1}\right)}^{L}\right)^{\lambda}\right)^{\frac{1}{\lambda}}}
\end{aligned}
$$

and also others are demonstrated similarly.

Therefore, we have GDIV P H FEW $A_{\epsilon}^{\omega(t)}\left(\widehat{\wp}\left(t_{1}\right)\right)=\left(\omega\left(t_{1}\right)\left(\widehat{\wp}\left(t_{1}\right)\right)^{\lambda}\right)^{\frac{1}{\lambda}}=\widehat{\wp}\left(t_{1}\right)$ and so Eq. (4.11) holds for $r=1$. 
- For $r=2$ :

By considering Eq. (4.12) for $k=1,2$, we have (from Definition 4.1 (c) and (d))

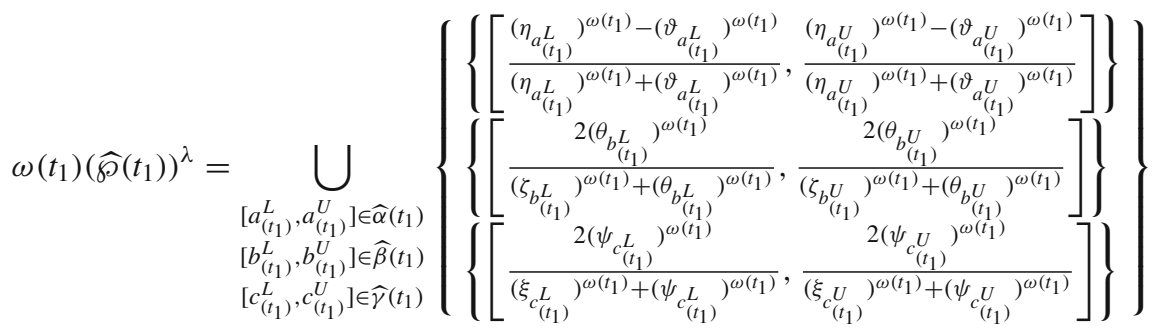

and

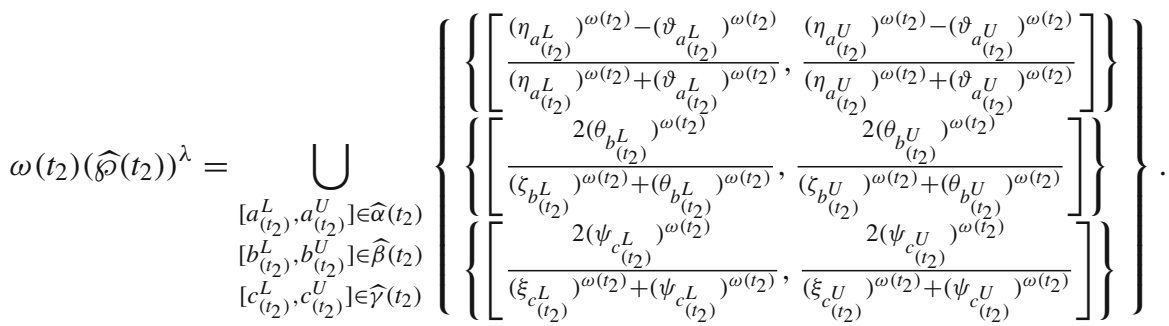

Then, we obtain that (from Definition 4.1 (a))

$$
\begin{aligned}
& \omega\left(t_{1}\right)\left(\widehat{\wp}\left(t_{1}\right)\right)^{\lambda} \oplus_{\epsilon} \omega\left(t_{2}\right)\left(\widehat{\wp}\left(t_{2}\right)\right)^{\lambda}
\end{aligned}
$$

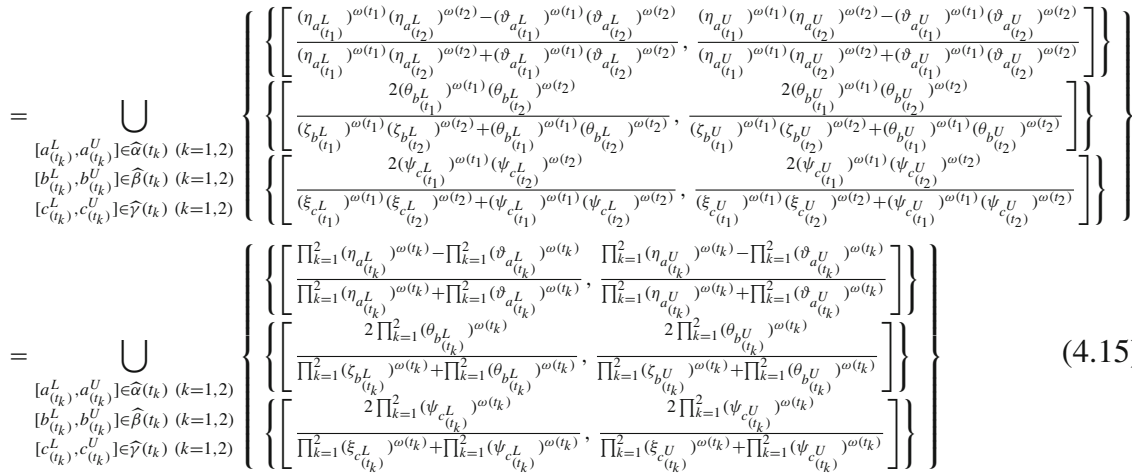

and thus

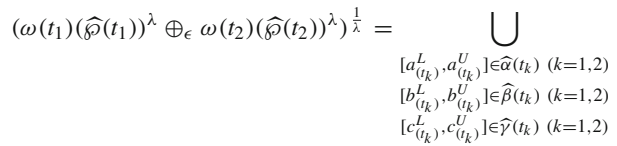




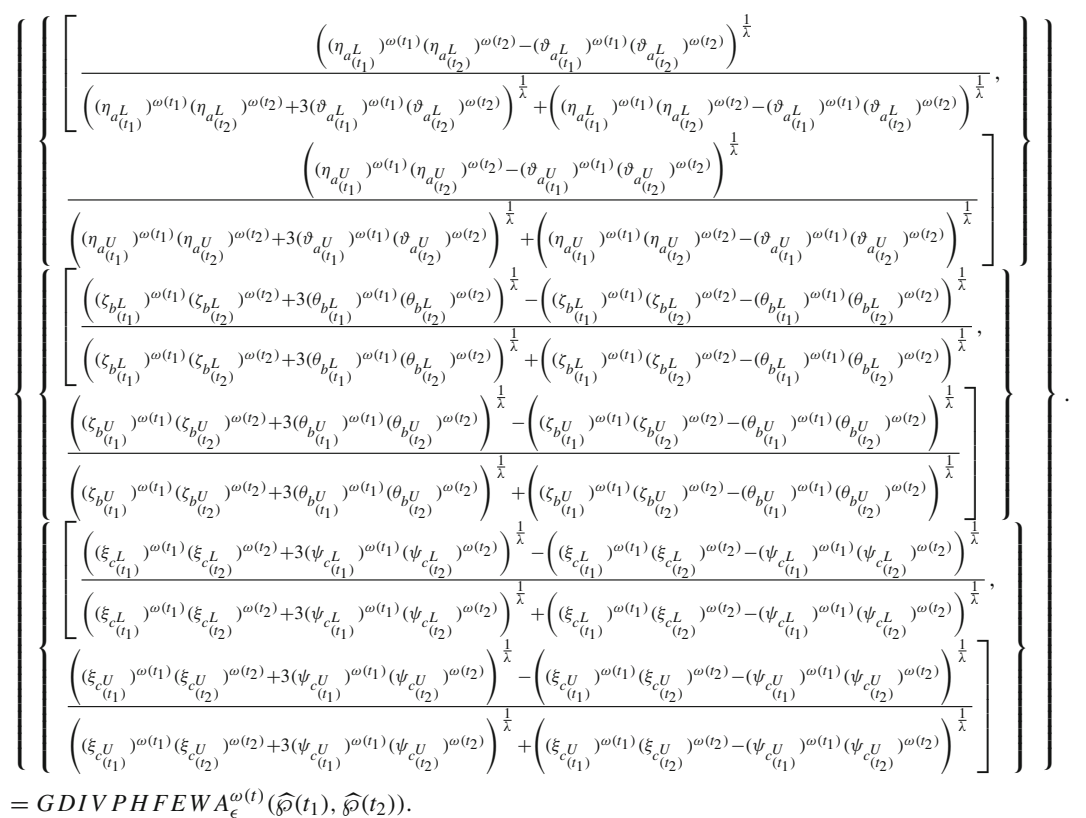

So, Eq. (4.11) is valid for $r=2$.

- For $r=s$ :

If Eq. (4.11) is valid, we have the following

GDIV P H FEW $A_{\epsilon}^{\omega(t)}\left(\widehat{\wp}\left(t_{1}\right), \widehat{\wp}\left(t_{2}\right), \ldots, \widehat{\wp}\left(t_{s}\right)\right)$

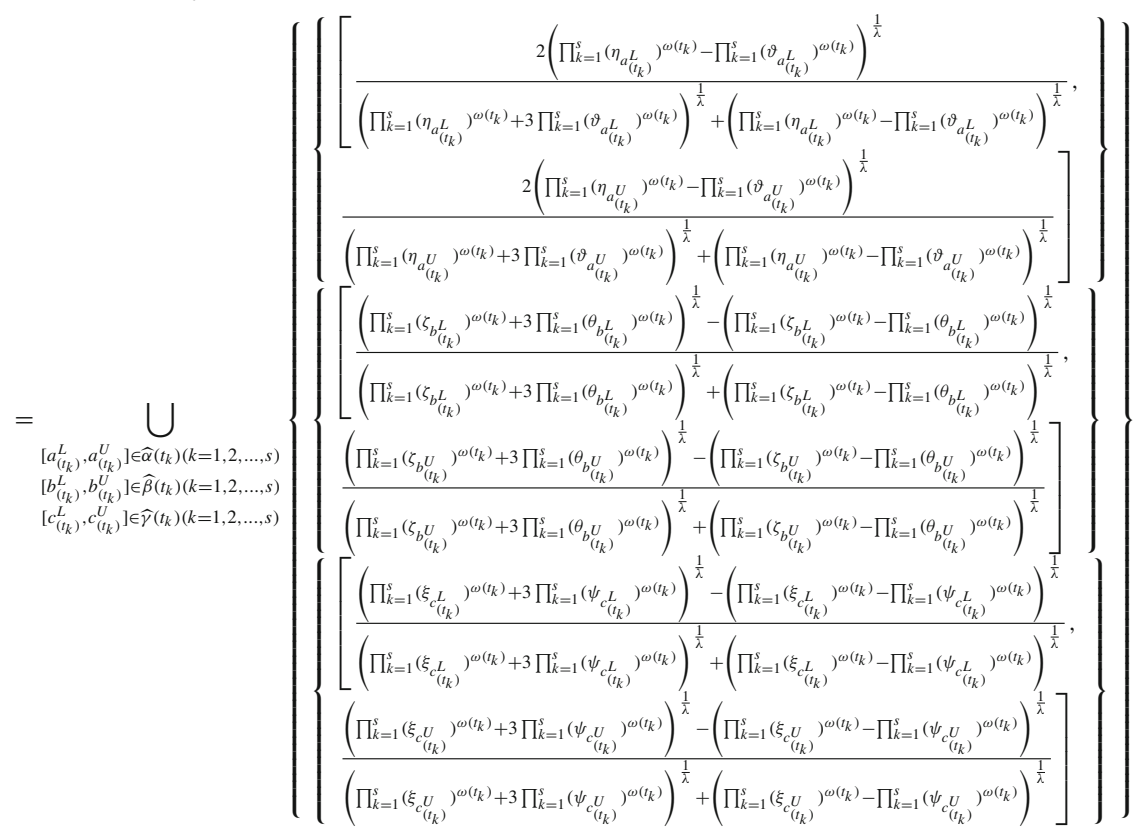

Now let's demonstrate that Eq. (4.11) holds when $r=s+1$.

In Eq. (4.15), $s$ is substituted for 2 and also by Eqs. (4.3), (4.4), we write

$\omega\left(t_{1}\right)\left(\widehat{\wp}\left(t_{1}\right)\right)^{\lambda} \oplus_{\epsilon} \omega\left(t_{2}\right)\left(\widehat{\wp}\left(t_{2}\right)\right)^{\lambda} \oplus_{\epsilon} \ldots \oplus_{\epsilon} \omega\left(t_{s}\right)\left(\widehat{\wp}\left(t_{s}\right)\right)^{\lambda} \oplus_{\epsilon} \omega\left(t_{s+1}\right)\left(\widehat{\wp}\left(t_{s+1}\right)\right)^{\lambda}$ 


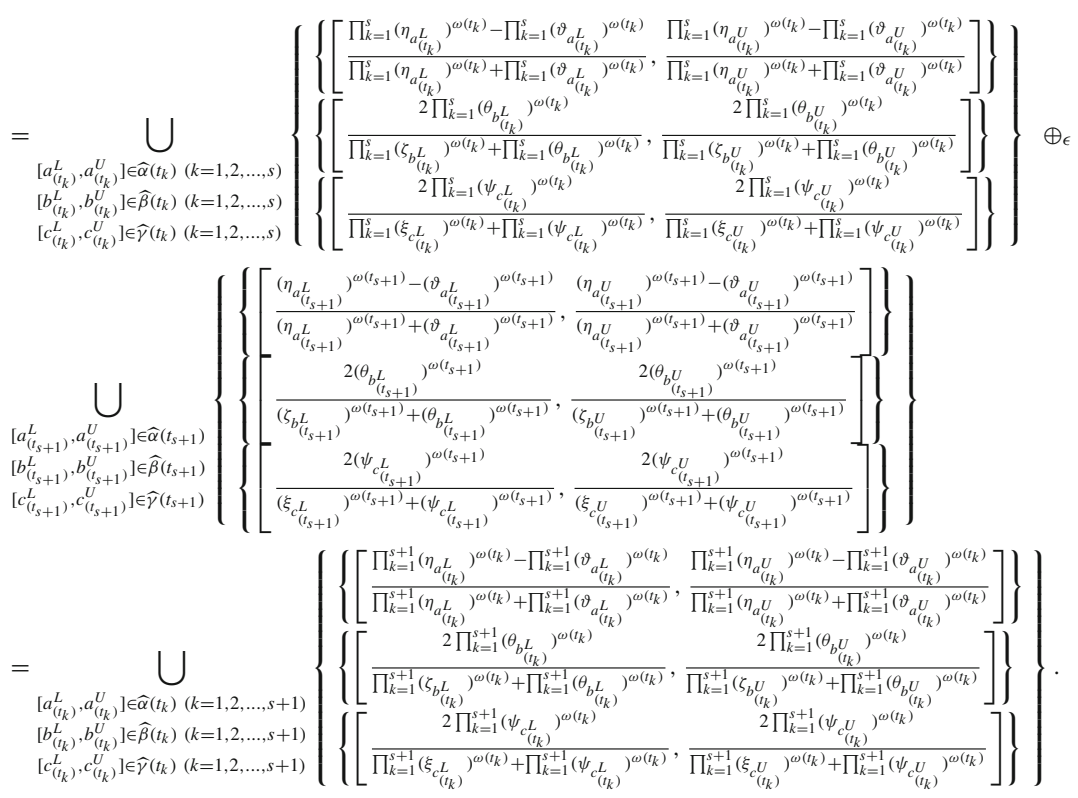

Then, we obtain that (from Definition 4.1 (d))

$$
\begin{aligned}
& \left(\omega\left(t_{1}\right)\left(\widehat{\wp}\left(t_{1}\right)\right)^{\lambda} \oplus_{\epsilon} \omega\left(t_{2}\right)\left(\widehat{\wp}\left(t_{2}\right)\right)^{\lambda} \oplus_{\epsilon} \ldots \oplus_{\epsilon} \omega\left(t_{s}\right)\left(\widehat{\wp}\left(t_{s}\right)\right)^{\lambda} \oplus_{\epsilon} \omega\left(t_{s+1}\right)\left(\widehat{\wp}\left(t_{s+1}\right)\right)^{\lambda}\right)^{\frac{1}{\lambda}}
\end{aligned}
$$

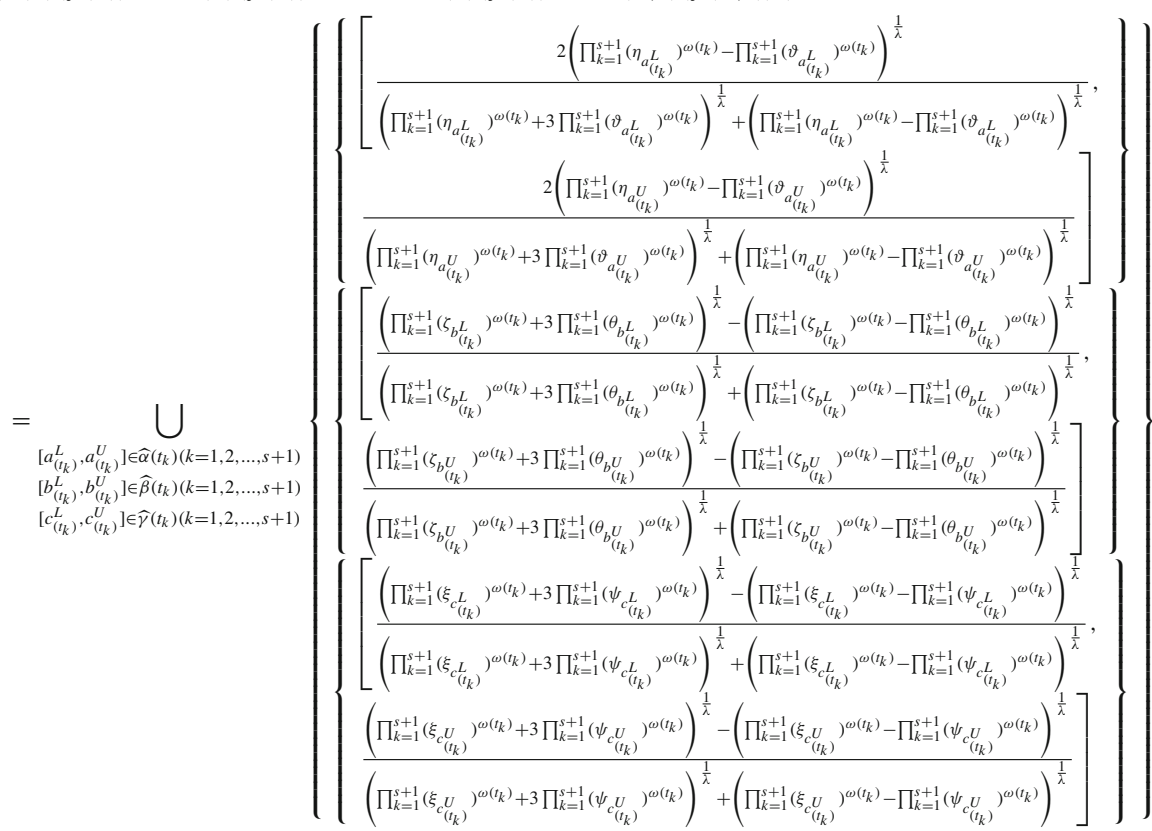

$=G D I V P H F E W A_{\epsilon}^{\omega(t)}\left(\widehat{\wp}\left(t_{1}\right), \widehat{\wp}\left(t_{2}\right), \ldots, \widehat{\wp}\left(t_{s+1}\right)\right)$

Thus, Eq. (4.11) is true for $r=s+1$.

Consequently, we say that Eq. (4.11) holds for all values of $r$. 
Note 3. If $\lambda=1$ then GDIVPHFEWA and GDIVPHFEWG operators reduce to DIVPHFEWA and DIVPHFEWG operators, respectively. Therefore, we will investigate the results of GDIPHFWA and GDIVPHFWG operators.

Theorem 4.6 (Idempotency) Let $\widehat{\wp}\left(t_{k}\right)(k=1,2, \ldots, r)$ be the IVPHFEs collected from $k$ different periods $t_{k}(k=1,2, \ldots, r)$. If $\widehat{\wp}\left(t_{k}\right)=\widehat{\wp}=\{\widehat{\alpha}, \widehat{\beta}, \widehat{\gamma}\}$ for all $k=1,2, \ldots, r$ then

1. GDIVPHFEW $A_{\omega(t)}\left(\widehat{\wp}\left(t_{1}\right), \widehat{\wp}\left(t_{2}\right), \ldots, \widehat{\wp}\left(t_{r}\right)\right)=\widehat{\wp}$.

2. GDIVPHFEW $G_{\omega(t)}\left(\widehat{\wp}\left(t_{1}\right), \widehat{\wp}\left(t_{2}\right), \ldots, \widehat{\wp}\left(t_{2}\right)\right)=\widehat{\wp}$.

Proof Let us prove GDIVPHFEW $A_{\omega(t)}\left(\widehat{\wp}\left(t_{1}\right), \widehat{\wp}\left(t_{2}\right), \ldots, \widehat{\wp}\left(t_{r}\right)\right)=\widehat{\wp}$, the other can be demonstrated similar to this.

Considering $\widehat{\wp}\left(t_{k}\right)=\widehat{\wp}=\{\widehat{\alpha}, \widehat{\beta}, \widehat{\gamma}\}$, by Theorem 4.5 (3) we have

$$
\begin{aligned}
& \text { GDIV P H FEW } A_{\omega(t)}\left(\widehat{\wp}\left(t_{1}\right), \widehat{\wp}\left(t_{2}\right), \ldots, \widehat{\wp}\left(t_{r}\right)\right) \\
& =\left(\left(\lambda\left(\widehat{\wp}\left(t_{1}\right)\right)^{\omega\left(t_{1}\right)} \oplus_{\epsilon} \lambda\left(\widehat{\wp}\left(t_{2}\right)\right)^{\omega\left(t_{2}\right)} \oplus_{\epsilon} \ldots \oplus_{\epsilon} \lambda\left(\widehat{\wp}\left(t_{r}\right)\right)^{\omega\left(t_{r}\right)}\right)\right)^{\frac{1}{\lambda}}
\end{aligned}
$$

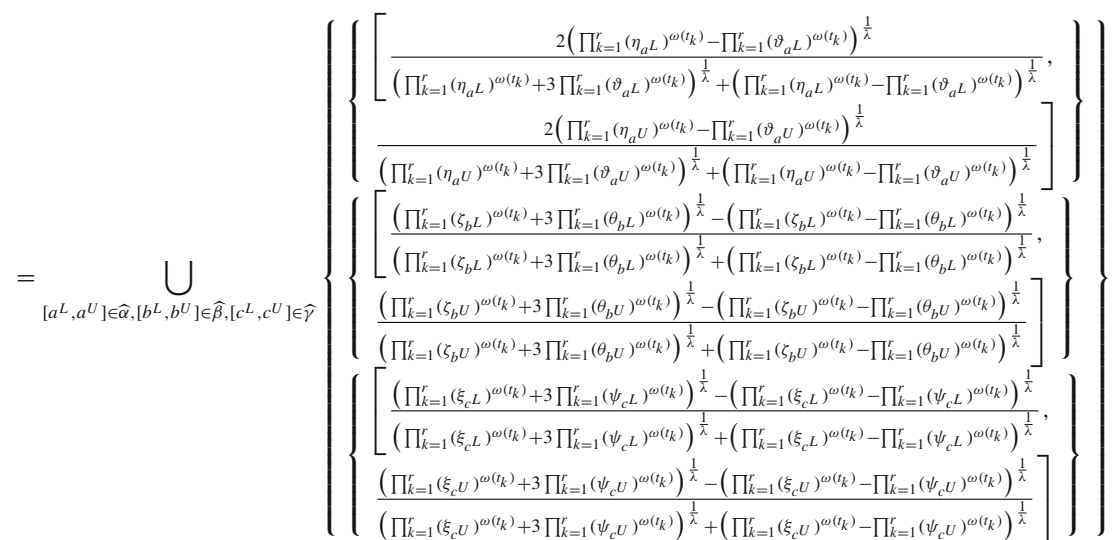

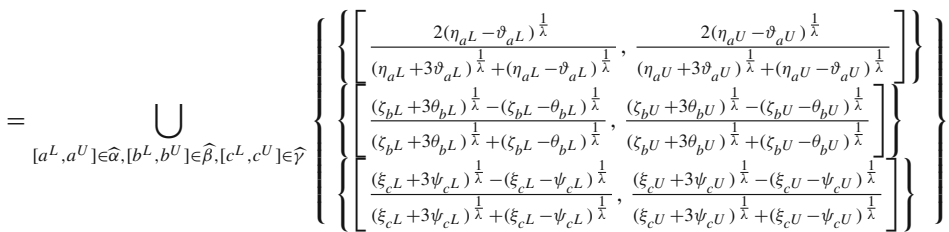

$$
\begin{aligned}
& =\underset{\left[a^{L}, a^{U}\right] \in \widehat{\alpha},\left[b^{L}, b^{U}\right] \in \widehat{\beta},\left[c^{L}, c^{U}\right] \in \widehat{\gamma}}{\bigcup}\left\{\begin{array}{l}
\left\{\left[a^{L}, a^{U}\right]\right\} \\
\left.\left[b^{L}, b^{U}\right]\right\} \\
\left.\left[c^{L}, c^{U}\right]\right\}
\end{array}\right\} \text { (by considering Eq. (4.12)) }
\end{aligned}
$$

Hence, the proof is completed.

Theorem 4.7 (Boundedness) Let $\widehat{\wp}\left(t_{k}\right)(k=1,2, \ldots, r)$ be the IVPHFEs collected from $k$ different periods $t_{k}(k=1,2, \ldots, r)$.

1. $\widehat{\wp}_{\min } \leq G D I V P H F E W A_{\omega(t)}\left(\widehat{\wp}\left(t_{1}\right), \widehat{\wp}\left(t_{2}\right), \ldots, \widehat{\wp}\left(t_{r}\right)\right) \leq \widehat{\wp}_{\max }$

2. $\widehat{\wp}_{\min } \leq G D I V P H F E W G_{\omega(t)}\left(\widehat{\wp}\left(t_{1}\right), \widehat{\wp}\left(t_{2}\right), \ldots, \widehat{\wp}\left(t_{r}\right)\right) \leq \widehat{\wp}_{\max }$

where

$$
\begin{aligned}
& \widehat{\wp}_{\min }=\left\{\widehat{\alpha}_{\min }, \widehat{\beta}_{\max }, \widehat{\gamma}_{\max }\right\}=\left\{\left\{\left[a_{\min }^{L}, a_{\min }^{U}\right]\right\},\left\{\left[b_{\max }^{L}, b_{\max }^{U}\right]\right\},\left\{\left[c_{\max }^{L}, c_{\max }^{U}\right]\right\}\right\}, \\
& \widehat{\wp}_{\max }=\left\{\widehat{\alpha}_{\max }, \widehat{\beta}_{\min }, \widehat{\gamma}_{\min }\right\}=\left\{\left\{\left[a_{\max }^{L}, a_{\max }^{U}\right]\right\},\left\{\left[b_{\min }^{L}, b_{\min }^{U}\right]\right\},\left\{\left[c_{\min }^{L}, c_{\min }^{U}\right]\right\}\right\}
\end{aligned}
$$


for

$$
\begin{aligned}
& a_{\min }^{L}=\bigcup_{\left[a_{\left(t_{k}\right)}^{L}, a_{\left(t_{k}\right)}^{U}\right] \in \widehat{\alpha}\left(t_{k}\right)} \min \left\{a_{\left(t_{k}\right)}^{L}\right\}, a_{\min }^{U}=\bigcup_{\left[a_{\left(t_{k}\right)}^{L}, a_{\left(t_{k}\right)}^{U}\right] \in \widehat{\alpha}\left(t_{k}\right)} \min \left\{a_{\left(t_{k}\right)}^{U}\right\}, \\
& a_{\max }^{L}=\bigcup_{\left[a_{\left(t_{k}\right)}^{L}, a_{\left(t_{k}\right)}^{U}\right] \in \widehat{\alpha}\left(t_{k}\right)} \max \left\{a_{\left(t_{k}\right)}^{L}\right\}, \\
& a_{\max }^{U}=\bigcup_{\left[a_{\left(t_{k}\right)}^{L}, a_{\left(t_{k}\right)}^{U}\right] \in \widehat{\alpha}\left(t_{k}\right)} \max \left\{a^{U}\right\}, b_{\min }^{L}=\bigcup_{\left[b_{\left(t_{k}\right)}^{L}, b_{\left(t_{k}\right)}^{U}\right] \in \widehat{\beta}\left(t_{k}\right)} \min \left\{b_{\left(t_{k}\right)}^{L}\right\}, \\
& b_{\min }^{U}=\bigcup_{\left[b_{\left(t_{k}\right)}^{L}, b_{\left(t_{k}\right)}^{U}\right] \in \widehat{\beta}\left(t_{k}\right)} \min \left\{b_{\left(t_{k}\right)}^{U}\right\}, \\
& b_{\max }^{L}=\bigcup_{\left[b_{\left(t_{k}\right)}^{L}, b_{\left(t_{k}\right)}^{U}\right] \in \widehat{\beta}\left(t_{k}\right)} \max \left\{b_{\left(t_{k}\right)}^{L}\right\}, b_{\max }^{U}=\bigcup_{\left[b_{\left(t_{k}\right)}^{L}, b_{\left(t_{k}\right)}^{U}\right] \in \widehat{\beta}\left(t_{k}\right)} \max \left\{b_{\left(t_{k}\right)}^{U}\right\}, \\
& c_{\min }^{L}=\bigcup_{\left[c_{\left(t_{k}\right)}^{L}, c_{\left(t_{k}\right)}^{U}\right] \in \widehat{\gamma}\left(t_{k}\right)} \min \left\{c_{\left(t_{k}\right)}^{L}\right\}, \\
& c_{\min }^{U}=\bigcup_{\left[c_{\left(t_{k}\right)}^{L}, c_{\left(t_{k}\right)}^{U}\right] \in \widehat{\gamma}\left(t_{k}\right)} \min \left\{c_{\left(t_{k}\right)}^{U}\right\}, c_{\max }^{L}=\bigcup_{\left[c_{\left(t_{k}\right)}^{L}, c_{\left(t_{k}\right)}^{U}\right] \in \widehat{\gamma}\left(t_{k}\right)} \max \left\{c_{\left(t_{k}\right)}^{L}\right\}, \\
& c_{\max }^{U}=\bigcup_{\left[c_{\left(t_{k}\right)}^{L}, c_{\left(t_{k}\right)}^{U}\right] \in \widehat{\gamma}\left(t_{k}\right)} \max \left\{c_{\left(t_{k}\right)}^{U}\right\} .
\end{aligned}
$$

Proof By considering Eqs. (4.11) and (4.13), they can be interpreted similar to the proof of Theorem 3.8.

Theorem 4.8 (Monotonicity) Let $\left(\widehat{\wp}\left(t_{1}\right), \widehat{\wp}\left(t_{2}\right), \ldots, \widehat{\wp}\left(t_{r}\right)\right)$ and $\left(\widehat{\wp}^{*}\left(t_{1}\right), \widehat{\wp}^{*}\left(t_{2}\right), \ldots, \widehat{\wp}^{*}\left(t_{r}\right)\right)$ be two collections of the IVPHFEs collected from $k$ different periods $t_{k}(k=1,2, \ldots, r)$. If $\widehat{\wp}\left(t_{k}\right) \leq \widehat{\wp}^{*}\left(t_{k}\right)$ for all $k=1,2, \ldots, r$ then

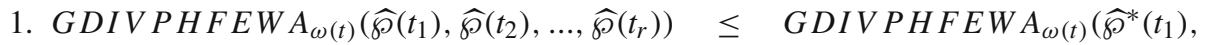
$\left.\widehat{\wp}^{*}\left(t_{2}\right), \ldots, \widehat{\wp}^{*}\left(t_{r}\right)\right)$.

2. GDIVPHFEWG $G_{\omega(t)}\left(\widehat{\wp}\left(t_{1}\right), \widehat{\wp}\left(t_{2}\right), \ldots, \widehat{\wp}\left(t_{r}\right)\right) \leq \operatorname{GDIVPHFEWG_{\omega (t)}}\left(\widehat{\wp}^{*}\left(t_{1}\right)\right.$, $\left.\widehat{\wp}^{*}\left(t_{2}\right), \ldots, \widehat{\wp}^{*}\left(t_{r}\right)\right)$.

Proof 1. The function $f(y)=\frac{(2-y)^{\lambda}-y^{\lambda}}{(2-y)^{\lambda}+3 y^{\lambda}}$ is the decreasing function, where $y \in[0,1]$ and $\lambda>0$. Since $\widehat{\wp}\left(t_{k}\right) \leq \widehat{\wp}^{*}\left(t_{k}\right)$ for all $k=1,2, \ldots, r$, we have $0 \leq a_{\left(t_{k}\right)}^{L} \leq a_{\left(t_{k}\right)}^{L^{*}}$ for all $k=1,2, \ldots, r$. Then, $f\left(a_{\left(t_{k}\right)}^{L}\right) \geq f\left(a_{\left(t_{k}\right)}^{L^{*}}\right)$ for all $k=1,2, \ldots, r$, i. e., $\frac{\left(2-a_{\left(t_{k}\right)}^{L}\right)^{\lambda}-\left(a_{\left(t_{k}\right)}^{L}\right)^{\lambda}}{\left(2-a_{\left(t_{k}\right)}^{L}\right)^{\lambda}+3\left(a_{\left(t_{k}\right)}^{L}\right)^{\lambda}} \geq$ $\frac{\left(2-a_{\left(t_{k}\right)}^{L^{*}}\right)^{\lambda}-\left(a_{\left(t_{k}\right)}^{L^{*}}\right)^{\lambda}}{\left(2-a_{\left(t_{k}\right)}^{L^{*}}\right)^{\lambda}+3\left(a_{\left(t_{k}\right)}^{L^{*}}\right)^{\lambda}} \Leftrightarrow \frac{\vartheta_{a_{\left(t_{k}\right)}^{L}}^{L}}{\eta_{a_{\left(t_{k}\right)}^{L}}^{L}} \geq \frac{\vartheta_{a_{\left(t_{k}\right)}^{L^{*}}}}{\eta_{a_{\left(t_{k}\right)}^{L^{*}}}}$ (where $\eta$ and $\vartheta$ are notations given in Eq. (4.12)).

Let $\omega(t)=\left(\omega\left(t_{1}\right), \omega\left(t_{2}\right), \ldots, \omega\left(t_{r}\right)\right)^{T}$ be the weighted vector of $t_{k}(k=1,2, \ldots, r)$, with $\omega\left(t_{k}\right) \in[0,1]$ and $\sum_{k=1}^{r} \omega\left(t_{k}\right)=1$. Then, we have

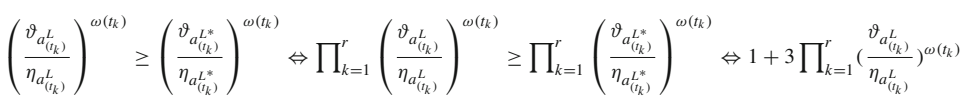

$$
\begin{aligned}
& \geq 1+3 \prod_{k=1}^{r}\left(\frac{\vartheta_{a_{\left(l^{*}\right)}}^{L^{*}}}{\eta_{a_{l_{k}}}^{L^{*}}}\right){ }^{\omega\left(t_{k}\right)}
\end{aligned}
$$


Dynamic aggregation operators and Einstein operations...

Page 31 of 52

127

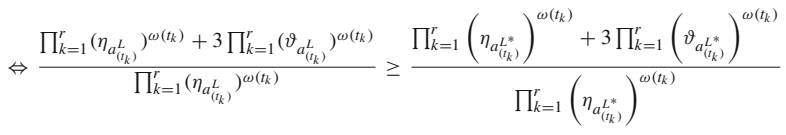

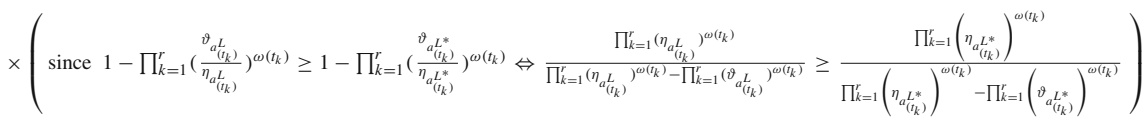

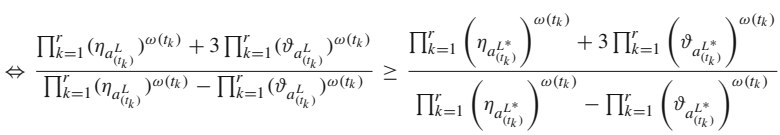

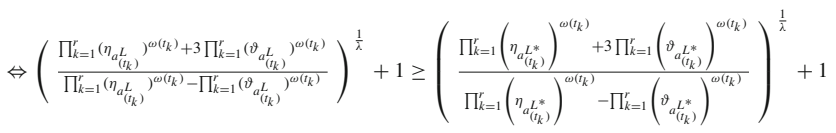

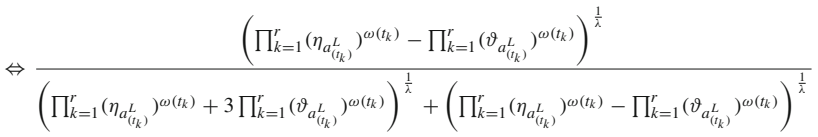

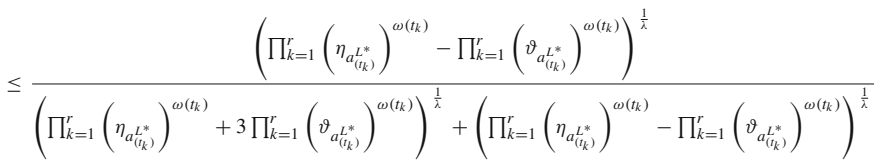

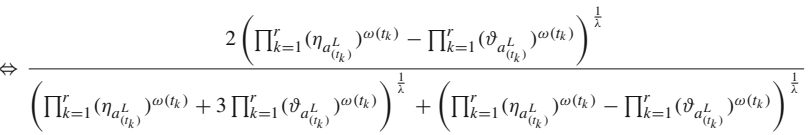

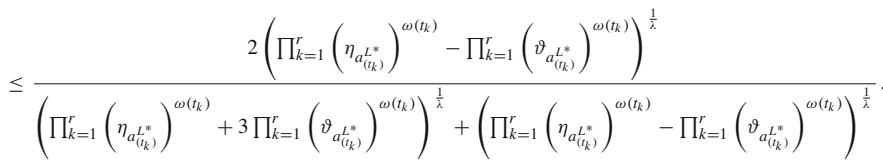

Likewise, it is obtained that

$$
\begin{gathered}
\Leftrightarrow \frac{2\left(\prod_{k=1}^{r}\left(\eta_{a_{\left(t_{k}\right)}^{U}}\right)^{\omega\left(t_{k}\right)}-\prod_{k=1}^{r}\left(\vartheta_{a_{\left(t_{k}\right)}^{U}}\right)^{\omega\left(t_{k}\right)}\right)^{\frac{1}{\lambda}}}{\left(\prod_{k=1}^{r}\left(\eta_{a_{\left(t_{k}\right)}^{U}}\right)^{\omega\left(t_{k}\right)}+3 \prod_{k=1}^{r}\left(\vartheta_{a_{\left(t_{k}\right)}^{U}}\right)^{\omega\left(t_{k}\right)}\right)^{\frac{1}{\lambda}}+\left(\prod_{k=1}^{r}\left(\eta_{a_{\left(t_{k}\right)}^{U}}\right)^{\omega\left(t_{k}\right)}-\prod_{k=1}^{r}\left(\vartheta_{a_{\left(t_{k}\right)}^{U}}\right)^{\omega\left(t_{k}\right)}\right)^{\frac{1}{\lambda}}} \\
\leq \frac{2\left(\prod_{k=1}^{r}\left(\eta_{a_{\left(t_{k}\right)}^{U *}}\right)^{\omega\left(t_{k}\right)}-\prod_{k=1}^{r}\left(\vartheta_{a_{\left(t_{k}\right)}^{U *}}\right)^{\omega\left(t_{k}\right)}\right)^{\frac{1}{\lambda}}}{\left(\prod_{k=1}^{r}\left(\eta_{a_{\left(t_{k}\right)}^{U^{*}}}\right)^{\omega\left(t_{k}\right)}+3 \prod_{k=1}^{r}\left(\vartheta_{a_{\left(t_{k}\right)}^{U^{*}}}\right)^{\omega\left(t_{k}\right)}\right)^{\frac{1}{\lambda}}+\left(\prod_{k=1}^{r}\left(\eta_{a_{\left(t_{k}\right)}^{U^{*}}}\right)^{\omega\left(t_{k}\right)}-\prod_{k=1}^{r}\left(\vartheta_{a_{\left(t_{k}\right)}^{U^{*}}}\right)^{\omega\left(t_{k}\right)}\right)^{\frac{1}{\lambda}}} .
\end{gathered}
$$

The function $g(z)=\frac{(1+z)^{\lambda}+3(1-z)^{\lambda}}{(1+z)^{\lambda}-(1-z)^{\lambda}}$ is the decreasing function, where $z \in(0,1]$ and $\lambda>$ 0 . Since $\widehat{\wp}\left(t_{k}\right) \leq \widehat{\wp}^{*}\left(t_{k}\right)$ for all $k=1,2, \ldots, r$, we have $b_{\left(t_{k}\right)}^{L} \geq b_{\left(t_{k}\right)}^{L^{*}}>0$ for all $k=$

Springer $S \mathrm{D} / \mathrm{VAC}$ 
$1,2, \ldots, r$. Then, $g\left(b_{\left(t_{k}\right)}^{L}\right) \leq g\left(b_{\left(t_{k}\right)}^{L^{*}}\right)$ for all $k=1,2, \ldots, r$, i. e., $\frac{\left(1+b_{\left(t_{k}\right)}^{L}\right)^{\lambda}+3\left(1-b_{\left(t_{k}\right)}^{L}\right)^{\lambda}}{\left(1+b_{\left(t_{k}\right)}^{L}\right)^{\lambda}-\left(1-b_{\left(t_{k}\right)}^{L}\right)^{\lambda}} \leq$ $\frac{\left(1+b_{\left(t_{k}\right)}^{L^{*}}\right)^{\lambda}+3\left(1-b_{\left(t_{k}\right)}^{L^{*}}\right)^{\lambda}}{\left(1+b_{\left(t_{k}\right)}^{L^{*}}\right)^{\lambda}-\left(1-b_{\left(t_{k}\right)}^{L^{*}}\right)^{\lambda}} \Leftrightarrow \frac{\zeta_{b_{\left(t_{k}\right)}^{L}}^{L}}{\theta_{b_{\left(t_{k}\right)}^{L}}^{L}} \leq \frac{\zeta_{b_{\left(t_{k}\right)}^{L^{*}}}}{\theta_{b_{\left(t_{k}\right)}^{L^{*}}}}$ (where $\zeta$ and $\theta$ are notations given in Eq. (4.12)).

Let $\omega(t)=\left(\omega\left(t_{1}\right), \omega\left(t_{2}\right), \ldots, \omega\left(t_{r}\right)\right)^{T}$ be the weighted vector of $t_{k}(k=1,2, \ldots, r)$, with $\omega\left(t_{k}\right) \in[0,1]$ and $\sum_{k=1}^{r} \omega\left(t_{k}\right)=1$. Then, we have

$$
\begin{aligned}
\left(\frac{\zeta_{b_{\left(t_{k}\right)}^{L}}}{\theta_{b_{\left(t_{k}\right)}^{L}}^{L}}\right)^{\omega\left(t_{k}\right)} & \leq\left(\frac{\zeta_{b_{\left(t_{k}\right)}^{L^{*}}}}{\theta_{b_{\left(t_{k}\right)}^{L^{*}}}}\right)^{\omega\left(t_{k}\right)} \Leftrightarrow\left(\frac{\theta_{b_{\left(t_{k}\right)}^{L}}}{\zeta_{b_{\left(t_{k}\right)}^{L}}^{L}}\right)^{\omega\left(t_{k}\right)} \geq\left(\frac{\theta_{b_{\left(t_{k}\right)}^{L^{*}}}}{\zeta_{b_{\left(t_{k}\right)} L^{*}}}\right)^{\omega\left(t_{k}\right)} \Leftrightarrow 1+3 \prod_{k=1}^{r}\left(\frac{\theta_{b_{\left(t_{k}\right)}^{L}}}{\zeta_{b_{\left(t_{k}\right)}^{L}}^{L}}\right)^{\omega\left(t_{k}\right)} \\
& \geq 1+3 \prod_{k=1}^{r}\left(\frac{\theta_{b_{\left(t_{k}\right)}^{L^{*}}}}{\zeta_{b_{\left(t_{k}\right)}^{L *}}^{L\left(t_{k}\right)}}\right)^{\omega}
\end{aligned}
$$

By proceeding similar to the calculations in (4.16), (4.17), (4.18), (4.19),

$$
\begin{aligned}
& \Leftrightarrow \frac{2\left(\prod_{k=1}^{r}\left(\zeta_{a_{\left(t_{k}\right)}^{L}}\right)^{\omega\left(t_{k}\right)}-\prod_{k=1}^{r}\left(\theta_{a_{\left(t_{k}\right)}^{L}}\right)^{\omega\left(t_{k}\right)}\right)^{\frac{1}{\lambda}}}{\left(\prod_{k=1}^{r}\left(\zeta_{a_{\left(t_{k}\right)}^{L}}\right)^{\omega\left(t_{k}\right)}+3 \prod_{k=1}^{r}\left(\theta_{a_{\left(k_{k}\right)}^{L}}\right)^{\omega\left(t_{k}\right)}\right)^{\frac{1}{\lambda}}+\left(\prod_{k=1}^{r}\left(\zeta_{a_{\left(l_{k}\right)}^{L}}\right)^{\omega\left(t_{k}\right)}-\prod_{k=1}^{r}\left(\theta_{a_{\left(k_{k}\right)}^{L}}\right)^{\omega\left(t_{k}\right)}\right)^{\frac{1}{\lambda}}} \\
& 2\left(\prod_{k=1}^{r}\left(\zeta_{a_{\left(k_{k}\right)}^{L^{*}}}\right)^{\omega\left(t_{k}\right)}-\prod_{k=1}^{r}\left(\theta_{a_{\left(t_{k}\right)}^{L^{*}}}\right)^{\omega\left(t_{k}\right)}\right)^{\frac{1}{\lambda}}
\end{aligned}
$$

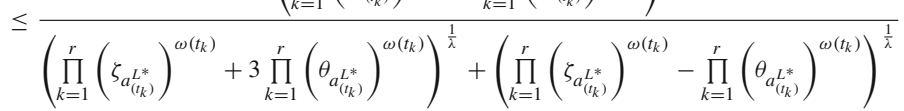

$$
\begin{aligned}
& 2\left(\prod_{k=1}^{r}\left(\zeta_{a_{\left(k_{k}\right)}^{L}}\right)^{\omega\left(t_{k}\right)}-\prod_{k=1}^{r}\left(\theta_{a_{\left(t_{k}\right)}^{L}}\right)^{\omega\left(t_{k}\right)}\right)^{\frac{1}{\lambda}} \\
& \Leftrightarrow 1-\frac{\left.\prod_{k=1}\left(\zeta_{a_{\left(k_{k}\right)}^{L}}\right)^{\omega\left(t_{k}\right)}+3 \prod_{k=1}^{r}\left(\theta_{a_{\left(k_{k}\right)}^{L}}\right)^{\omega\left(t_{k}\right)}\right)^{\frac{1}{\lambda}}+\left(\prod_{k=1}^{r}\left(\zeta_{a_{\left(k_{k}\right)}^{L}}\right)^{\omega\left(t_{k}\right)}-\prod_{k=1}^{r}\left(\theta_{a_{\left(k_{k}\right)}^{L}}\right)^{\omega\left(t_{k}\right)}\right)^{\frac{1}{\lambda}}}{\left(\prod^{2}\right.}
\end{aligned}
$$

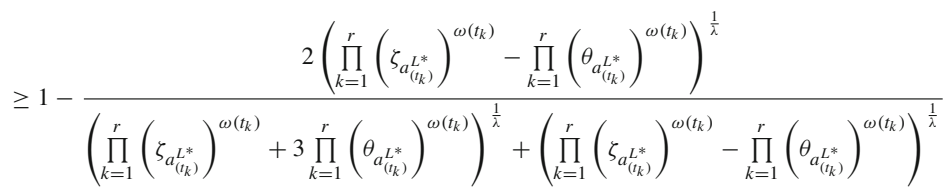

$$
\begin{aligned}
& \Leftrightarrow \frac{\left(\prod_{k=1}^{r}\left(\zeta_{b_{\left(t_{k}\right)}^{L}}\right)^{\omega\left(t_{k}\right)}+3 \prod_{k=1}^{r}\left(\theta_{b_{\left(k_{k}\right)}^{L}}\right)^{\omega\left(t_{k}\right)}\right)^{\frac{1}{\lambda}}-\left(\prod_{k=1}^{r}\left(\zeta_{b_{\left(t_{k}\right)}^{L}}\right)^{\omega\left(t_{k}\right)}-\prod_{k=1}^{r}\left(\theta_{b_{\left(t_{k}\right)}^{L}}\right)^{\omega\left(t_{k}\right)}\right)^{\frac{1}{\lambda}}}{\left(\prod_{k=1}^{r}\left(\zeta_{b_{\left(t_{k}\right)}^{L}}\right)^{\omega\left(t_{k}\right)}+3 \prod_{k=1}^{r}\left(\theta_{b_{\left(k_{k}\right)}^{L}}\right)^{\omega\left(t_{k}\right)}\right)^{\frac{1}{\lambda}}+\left(\prod_{k=1}^{r}\left(\zeta_{b_{\left(k_{k}\right)}^{L}}\right)^{\omega\left(t_{k}\right)}-\prod_{k=1}^{r}\left(\theta_{\left.b_{\left(t_{k}\right)}^{L}\right)}\right)^{\omega\left(t_{k}\right)}\right)^{\frac{1}{\lambda}}} \\
& \geq \frac{\left(\prod_{k=1}^{r}\left(\zeta_{b_{\left(t_{k}\right)}^{L *}}\right)^{\omega\left(t_{k}\right)}+3 \prod_{k=1}^{r}\left(\theta_{b_{\left(t_{k}\right)}^{L^{*}}}\right)^{\omega\left(t_{k}\right)}\right)^{\frac{1}{\lambda}}-\left(\prod_{k=1}^{r}\left(\zeta_{L_{\left(t_{k}\right)}^{L^{*}}}\right)^{\omega\left(t_{k}\right)}-\prod_{k=1}^{r}\left(\theta_{b_{\left(t_{k}\right)}^{L^{*}}}\right)^{\omega\left(t_{k}\right)}\right)^{\frac{1}{\lambda}}}{\left(\prod_{k=1}^{r}\left(\zeta_{b_{\left(t_{k}\right)}^{L^{*}}}\right)^{\omega\left(t_{k}\right)}+3 \prod_{k=1}^{r}\left(\theta_{b_{\left(t_{k}\right)}^{L^{*}}}\right)^{\omega\left(t_{k}\right)}\right)^{\frac{1}{\lambda}}+\left(\prod_{k=1}^{r}\left(\zeta_{b_{\left(k_{k}\right)}^{L^{*}}}\right)^{\omega\left(t_{k}\right)}-\prod_{k=1}^{r}\left(\theta_{b_{\left(t_{k}\right)}^{L^{*}}}\right)^{\omega\left(t_{k}\right)}\right)^{\frac{1}{\lambda}}} .
\end{aligned}
$$

Note that Eq. (4.24) is valid even if $b_{\left(t_{k}\right)}^{L}=b_{\left(t_{k}\right)}^{L^{*}}=0$ for all $k=1,2, \ldots, r$. 
Likewise, it is obtained that

$$
\begin{aligned}
& \Leftrightarrow \frac{\left(\prod_{k=1}^{r}\left(\zeta_{b_{(k)}}^{U}\right)^{\omega\left(t_{k}\right)}+3 \prod_{k=1}^{r}\left(\theta_{b_{\left(k_{k}\right)}^{U}}\right)^{\omega\left(t_{k}\right)}\right)^{\frac{1}{\lambda}}-\left(\prod_{k=1}^{r}\left(\zeta_{b_{(k)}}^{U}\right)^{\omega\left(t_{k}\right)}-\prod_{k=1}^{r}\left(\theta_{b_{\left(k_{k}\right)}^{U}}\right)^{\omega\left(t_{k}\right)}\right)^{\frac{1}{\lambda}}}{\left(\prod_{k=1}^{r}\left(\zeta_{b_{(k)}}^{U}\right)^{\omega\left(t_{k}\right)}+3 \prod_{k=1}^{r}\left(\theta_{b_{\left(k_{k}\right)}^{U}}\right)^{\omega\left(t_{k}\right)}\right)^{\frac{1}{\lambda}}+\left(\prod_{k=1}^{r}\left(\zeta_{b_{\left(k_{k}\right)}^{U}}\right)^{\omega\left(t_{k}\right)}-\prod_{k=1}^{r}\left(\theta_{b_{\left(k_{k}\right)}^{U}}\right)^{\omega\left(t_{k}\right)}\right)^{\frac{1}{\lambda}}}
\end{aligned}
$$

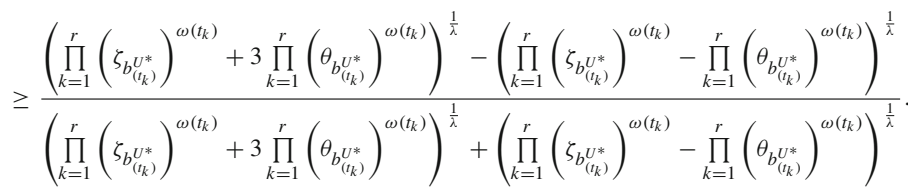

Similarly, by considering the above function $g$, we have that

$$
\begin{aligned}
& \Leftrightarrow \frac{\left(\prod_{k=1}^{r}\left(\xi_{c_{\left(t_{k}\right)}^{L}}\right)^{\omega\left(t_{k}\right)}+3 \prod_{k=1}^{r}\left(\psi_{c_{\left(k_{k}\right)}^{L}}\right)^{\omega\left(t_{k}\right)}\right)^{\frac{1}{\lambda}}-\left(\prod_{k=1}^{r}\left(\xi_{c_{\left(t_{k}\right)}^{L}}\right)^{\omega\left(t_{k}\right)}-\prod_{k=1}^{r}\left(\psi_{c_{\left(k_{k}\right)}^{L}}\right)^{\omega\left(t_{k}\right)}\right)^{\frac{1}{\lambda}}}{\left(\prod_{k=1}^{r}\left(\xi_{c_{\left(t_{k}\right)}^{L}}\right)^{\omega\left(t_{k}\right)}+3 \prod_{k=1}^{r}\left(\psi_{c_{\left(k_{k}\right)}^{L}}\right)^{\omega\left(t_{k}\right)}\right)^{\frac{1}{\lambda}}+\left(\prod_{k=1}^{r}\left(\xi_{c_{\left(t_{k}\right)}^{L}}\right)^{\omega\left(t_{k}\right)}-\prod_{k=1}^{r}\left(\psi_{c_{\left(t_{k}\right)}^{L}}^{L}\right)^{\omega\left(t_{k}\right)}\right)^{\frac{1}{\lambda}}} \\
& \geq \frac{\left(\prod_{k=1}^{r}\left(\xi_{c_{\left(t_{k}\right)}^{L^{*}}}{ }^{\omega\left(t_{k}\right)}+3 \prod_{k=1}^{r}\left(\psi_{c_{\left(t_{k}\right)}^{L^{*}}}\right)^{\omega\left(t_{k}\right)}\right)^{\frac{1}{\lambda}}-\left(\prod_{k=1}^{r}\left(\xi_{c_{\left(t_{k}\right)}^{L^{*}}}\right)^{\omega\left(t_{k}\right)}-\prod_{k=1}^{r}\left(\psi_{c_{\left(t_{k}\right)}^{L^{*}}}\right)^{\omega\left(t_{k}\right)}\right)^{\frac{1}{\lambda}}\right.}{\left(\prod_{k=1}^{r}\left(\xi_{c_{\left(k_{k}\right)}}\right)^{\omega\left(t_{k}\right)}+3 \prod_{k=1}^{r}\left(\psi_{c_{\left(t_{k}\right)}^{L^{*}}}\right)^{\omega\left(t_{k}\right)}\right)^{\frac{1}{\lambda}}+\left(\prod_{k=1}^{r}\left(\xi_{c_{\left(t_{k}\right)}^{L^{*}}}\right)^{\omega\left(t_{k}\right)}-\prod_{k=1}^{r}\left(\psi_{c_{\left(k_{k}\right)}}\right)^{\omega\left(t_{k}\right)}\right)^{\frac{1}{\lambda}}}
\end{aligned}
$$

and

$$
\begin{aligned}
& \Leftrightarrow \frac{\left(\prod_{k=1}^{r}\left(\xi_{c_{\left(t_{k}\right)}^{U}}\right)^{\omega\left(t_{k}\right)}+3 \prod_{k=1}^{r}\left(\psi_{c_{\left(t_{k}\right)}^{U}}\right)^{\omega\left(t_{k}\right)}\right)^{\frac{1}{\lambda}}-\left(\prod_{k=1}^{r}\left(\xi_{c_{\left(t_{k}\right)}^{U}}\right)^{\omega\left(t_{k}\right)}-\prod_{k=1}^{r}\left(\psi_{c_{\left(t_{k}\right)}^{U}}\right)^{\omega\left(t_{k}\right)}\right)^{\frac{1}{\lambda}}}{\left(\prod_{k=1}^{r}\left(\xi_{c_{\left(t_{k}\right)}^{U}}\right)^{\omega\left(t_{k}\right)}+3 \prod_{k=1}^{r}\left(\psi_{c_{\left(t_{k}\right)}^{U}}\right)^{\omega\left(t_{k}\right)}\right)^{\frac{1}{\lambda}}+\left(\prod_{k=1}^{r}\left(\xi_{c_{\left(t_{k}\right)}^{U}}\right)^{\omega\left(t_{k}\right)}-\prod_{k=1}^{r}\left(\psi_{c_{\left(t_{k}\right)}^{U}}\right)^{\omega\left(t_{k}\right)}\right)^{\frac{1}{\lambda}}} \\
& \geq \frac{\left(\prod_{k=1}^{r}\left(\xi_{c_{\left(t_{k}\right)}}\right)^{\omega\left(t_{k}\right)}+3 \prod_{k=1}^{r}\left(\psi_{c_{\left(t_{k}\right)}^{U^{*}}}\right)^{\omega\left(t_{k}\right)}\right)^{\frac{1}{\lambda}}-\left(\prod_{k=1}^{r}\left(\xi_{c_{\left(t_{k}\right)}^{U^{*}}}\right)^{\omega\left(t_{k}\right)}-\prod_{k=1}^{r}\left(\psi_{c_{\left(t_{k}\right)}^{U}}\right)^{\omega\left(t_{k}\right)}\right)^{\frac{1}{\lambda}}}{\left(\prod_{k=1}^{r}\left(\xi_{c_{\left(t_{k}\right)}}\right)^{\omega\left(t_{k}\right)}+3 \prod_{k=1}^{r}\left(\psi_{c_{\left(t_{k}\right)}^{U^{*}}}\right)^{\omega\left(t_{k}\right)}\right)^{\frac{1}{\lambda}}+\left(\prod_{k=1}^{r}\left(\xi_{c_{\left(t_{k}\right)}^{U^{*}}}\right)^{\omega\left(t_{k}\right)}-\prod_{k=1}^{r}\left(\psi_{c_{\left(t_{k}\right)}}\right)^{\omega\left(t_{k}\right)}\right)^{\frac{1}{\lambda}}} .
\end{aligned}
$$

Thus, the proof of (1) is completed.

2. It can be shown similar to (1), therefore, omitted.

Theorem 4.9 (Commutativity) Let $\widehat{\wp}\left(t_{1}\right), \widehat{\wp}\left(t_{2}\right), \ldots, \widehat{\wp}\left(t_{r}\right)$ be a collection of the IVPHFEs collected from $k$ different periods $t_{k}(k=1,2, \ldots, r)$. In addition, let $\widehat{\wp_{n}}\left(t_{1_{n}}\right), \widehat{\wp_{n}}\left(t_{2_{n}}\right), \ldots, \widehat{\wp_{n}}\left(t_{r_{n}}\right)$ be a new permutation of $\widehat{\wp}\left(t_{1}\right), \widehat{\wp}\left(t_{2}\right), \ldots, \widehat{\wp}\left(t_{r}\right)$ then

1. GDIVPHFEWA $A_{\omega(t)}\left(\widehat{\wp}\left(t_{1}\right), \widehat{\wp}\left(t_{2}\right), \ldots, \widehat{\wp}\left(t_{r}\right)\right)=G D I V P H F E W A_{\omega(t)}\left(\widehat{\wp n}\left(t_{1_{n}}\right)\right.$, $\left.\widehat{\wp_{n}}\left(t_{2_{n}}\right), \ldots, \widehat{\wp_{n}}\left(t_{r_{n}}\right)\right)$.

2. GDIVPHFEWG $G_{\omega(t)}\left(\widehat{\wp}\left(t_{1}\right), \widehat{\wp}\left(t_{2}\right), \ldots, \widehat{\wp}\left(t_{r}\right)\right)=G D I V P H F E W G_{\omega(t)}\left(\widehat{\wp n}\left(t_{1_{n}}\right)\right.$, $\left.\widehat{\wp_{n}}\left(t_{2_{n}}\right), \ldots, \widehat{\wp_{n}}\left(t_{r_{n}}\right)\right)$.

Proof It can be easily seen by Theorem 4.5 (3) and (4). 


\section{The approaches to multi-period decision making under IVPHF environments}

The MCDM is an important part of modern decision science. It has been extensively applied to various areas such as economics, society, management, military, etc., and has been receiving more and more attention in recent years. To date, however, most research has focused on single-period MCDM in which all the original decision information is collected at the same period and several methods have been proposed to solve this kind of problem. However, some complex DM problems have to consider the current and past performance of alternatives. This kind of DMs is called multi-period decision making (MPDM) and the original decision information is usually collected at different periods or refers to different moments in time. When the decision-making information takes form as interval-valued picture hesitant fuzzy information and is collected at different periods, the dynamic interval-valued picture hesitant fuzzy (Einstein) aggregation operators are suitable for dealing with such situations. In this part, we develop two approaches, using the proposed dynamic interval-valued picture hesitant fuzzy aggregation operators and dynamic interval-valued picture hesitant fuzzy Einstein aggregation operators, to multi-period multi-criteria decision making under the intervalvalued picture hesitant fuzzy information.

The following notations are utilized to depict the possible numerical problems:

Let $Q=\left\{q_{1}, q_{2}, \ldots, q_{n}\right\}$ be a set of alternatives, and $L=\left\{\ell_{1}, \ell_{2}, \ldots, \ell_{m}\right\}$ be a set of parameters (criteria) and its weight vector be $\omega=\left(\omega_{1}, \omega_{2}, \ldots, \omega_{m}\right)^{T}$, where $\omega_{j} \in[0,1]$ $(j=1,2, \ldots, m), \sum_{j=1}^{m} \omega_{j}=1$. In addition, $\mathcal{T}=\left\{t_{1}, t_{2}, \ldots, t_{r}\right\}$ be a set of time variables (or periods) and its weight vector be $\omega(t)=\left(\omega\left(t_{1}\right), \omega\left(t_{2}\right), \ldots, \omega\left(t_{r}\right)\right)^{T}$ where $\omega\left(t_{k}\right) \in[0,1]$, $\sum_{k=1}^{r} \omega\left(t_{k}\right)=1$.

Assume that the IVPHF decision matrix created by the expert (decision maker) at the periods $t_{k}(k=1,2, \ldots, r)$ to measure the degrees of the alternative $q_{i}(i=1,2, \ldots, n)$ satisfies the parameter $\ell_{j}(j=1,2, \ldots, m)$ is $D\left(t_{k}\right)=\left(d_{i j}\left(t_{k}\right)\right)_{n \times m}$ where

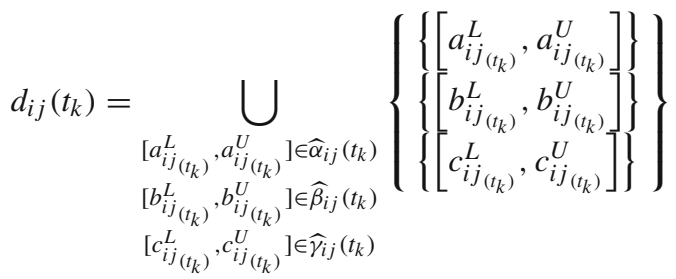

for $i=1,2, \ldots, n, j=1,2, \ldots, m$ and $k=1,2, \ldots, r$.

\section{Algorithm 1.}

Step 1. Construct the decision matrix $D\left(t_{k}\right)=\left(d_{i j}\left(t_{k}\right)\right)_{n \times m}$ and construct the idealized decision matrix $\mathfrak{D}\left(t_{k}\right)=\left(\mathfrak{d}_{i j}\left(t_{k}\right)\right)_{n \times m}$.

Generally, there are two types for the criteria: benefit criteria (such criterion has a positive effect on the decision and the value of IVPHFE(s) is taken as is) and cost criteria (such criterion has a negative effect on the decision and the complement of the value of IVPHFE(s) is taken to include this criterion in the decision). We may transform the criterion values from cost type to benefit type: in such a case, the IVPHF decision matrix $D\left(t_{k}\right)=\left(d_{i j}\left(t_{k}\right)\right)_{n \times m}$ can be transformed into matrix 
$\mathfrak{D}\left(t_{k}\right)=\left(\mathfrak{d}_{i j}\left(t_{k}\right)\right)_{n \times m}$ where for $i=1,2, \ldots, n, j=1,2, \ldots, m$ and $k=1,2, \ldots, r$

$$
\begin{aligned}
& \mathfrak{d}_{i j}\left(t_{k}\right)=\bigcup_{\left[\tilde{a}_{i j_{\left(t_{k}\right)}}^{L} \tilde{a}_{i j_{\left(t_{k}\right)}}^{U}\right] \in \tilde{\alpha}_{i j}\left(t_{k}\right)}\left\{\begin{array}{l}
\left\{\left[\tilde{a}_{i j_{\left(t_{k}\right)}}^{L}, \tilde{a}_{i j_{\left(t_{k}\right)}}^{U}\right]\right\} \\
\left\{\left[\tilde{b}_{i j_{\left(t_{k}\right)}}^{L}, \tilde{b}_{i j_{\left(t_{k}\right)}}^{U}\right]\right\} \\
\left\{\left[\tilde{c}_{i j_{\left(t_{k}\right)}}^{L}, \tilde{c}_{i j_{\left(t_{k}\right)}}^{U}\right]\right\}
\end{array}\right\} \\
& {\left[\tilde{b}_{i j}^{L}{ }_{\left(t_{k}\right)} \tilde{b}_{i j}^{U}{ }_{\left(t_{k}\right)}\right] \in \widetilde{\beta}_{i j}\left(t_{k}\right)} \\
& {\left[\tilde{c}_{i{ }_{\left(t_{k}\right)}}^{L}, \tilde{c}_{i j_{\left(t_{k}\right)}}^{U}\right] \in \tilde{\gamma}_{i j}\left(t_{k}\right)}
\end{aligned}
$$

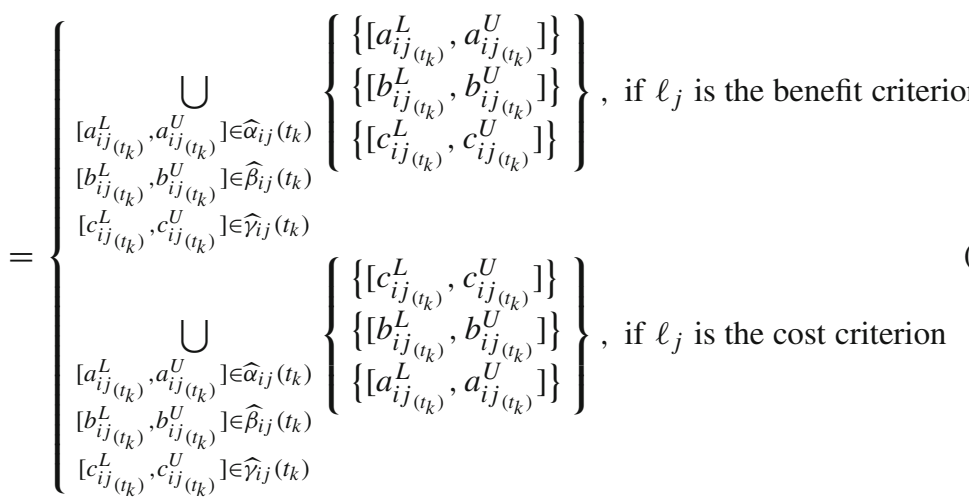

Step 2. Utilize the dynamic interval-valued picture hesitant fuzzy aggregation operators (DIVPHFWA, DIVPHFWG, GDIVPHFWA, GDIVPHFWG) to aggregate all the idealized decision matrix $\left(\mathfrak{d}_{i j}\left(t_{k}\right)\right)_{n \times m}(k=1,2, \ldots, r)$ into the collective decision matrix $\left(\mathfrak{d}_{i j}\right)_{n \times m}$.

If we use the GDIVPHFWG operator then we take for $i=1,2, \ldots, n$ and $j=$ $1,2, \ldots, m$

$$
\begin{aligned}
& \mathfrak{d}_{i j}=G D I V P H F W G_{\omega(t)}\left(\mathfrak{d}_{i j}\left(t_{k}\right) ; k=1,2, \ldots, r\right)
\end{aligned}
$$

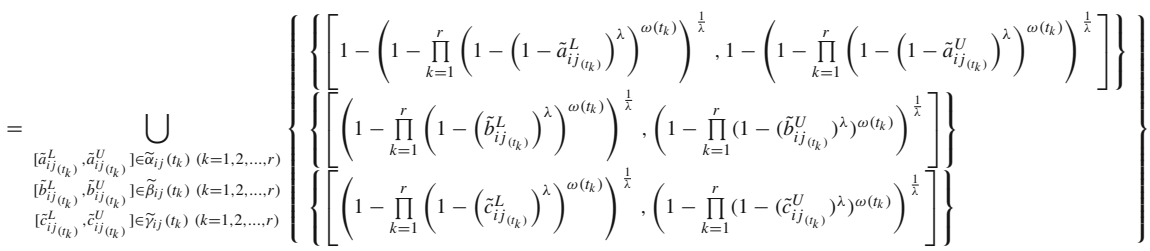

Step 3. Utilize the interval-valued picture hesitant fuzzy aggregation operators (IVPHFWA, IVPHFWG) to find the collective overall preference values $\mathfrak{d}_{i}(i=1,2, \ldots, n)$ of the alternatives $q_{i}(i=1,2, \ldots, n)$.

If we use the IVPHFWG operator then we take for $i=1,2, \ldots, n$

$$
\begin{aligned}
& \mathfrak{d}_{i}=I V P H F W G_{\omega}\left(\mathfrak{d}_{i j} ; j=1,2, \ldots, m\right) \\
& =\underset{\substack{\left[\tilde{a}_{j i}^{L}, \tilde{a}_{i j}^{U}\right] \in \widetilde{\alpha}_{i j}(j=1,2, \ldots, m) \\
\left[\tilde{b}_{i j}^{L}, \tilde{b}_{i j}^{U}\right] \in \tilde{\beta}_{i j}(j=1,2, \ldots, m) \\
\left[\tilde{c}_{i j}^{L}, \tilde{c}_{i j}^{U}\right] \in \widetilde{\gamma}_{i j}(j=1,2, \ldots, m)}}{\bigcup}\left\{\left\{\left\{\left[\begin{array}{l}
\left.\left.\prod_{j=1}^{m}\left(\tilde{a}_{i j}^{L}\right)^{\omega_{j}}, \prod_{j=1}^{m}\left(\tilde{a}_{i j}^{U}\right)^{\omega_{j}}\right]\right\} \\
\left.\left.1-\prod_{j=1}^{m}\left(1-\tilde{b}_{i j}^{L}\right)^{\omega_{j}}, 1-\prod_{j=1}^{m}\left(1-\tilde{b}_{i j}^{U}\right)^{\omega_{j}}\right]\right\} \\
1-\prod_{j=1}^{m}\left(1-\tilde{c}_{i j}^{L}\right)^{\omega_{j}}, 1-\prod_{j=1}^{m}\left(1-\tilde{c}_{i j}^{U}\right)^{\omega_{j}}
\end{array}\right]\right\}\right\} .\right.
\end{aligned}
$$


Step 4. Calculate the score values $\mathcal{S}\left(\mathfrak{d}_{i}\right)$ of $\mathfrak{d}_{i}(i=1,2, \ldots, n)$.

When two score values $\mathcal{S}\left(\mathfrak{d}_{i}\right)$ and $\mathcal{S}\left(\mathfrak{d}_{i^{\prime}}\right)$ are equal, calculate the accuracy values $\mathcal{A}\left(\mathfrak{d}_{i}\right)$ and $\mathcal{A}\left(\mathfrak{d}_{i^{\prime}}\right)$. If $\mathcal{A}\left(\mathfrak{d}_{i}\right)$ and $\mathcal{A}\left(\mathfrak{d}_{i^{\prime}}\right)$ are qual, calculate the certainty values $\mathcal{C}\left(\mathfrak{d}_{i}\right)$ and $\mathcal{C}\left(\mathfrak{d}_{i^{\prime}}\right)$.

Step 5. Rank all the alternatives $\left\{q_{1}, q_{2}, \ldots, q_{n}\right\}$ and select the optimal one(s) by the score values (the accuracy values in the case of equality of score values, the certainty values in the case of equality of accuracy values).

\section{Algorithm 2.}

Step 1. Same as Step 1 of Algorithm 1.

Step 2. Utilize the dynamic interval-valued picture hesitant fuzzy Einstein aggregation operators (DIVPHFEWA, DIVPHFEWG, GDIVPHFEWA, GDIVPHFEWG) to aggregate all the idealized decision matrix $\left(\mathfrak{d}_{i j}\left(t_{k}\right)\right)_{n \times m}(k=1,2, \ldots, r)$ into the collective decision matrix $\left(\mathfrak{d}_{i j}\right)_{n \times m}$.

If we use the GDIVPHFEWA operator then we take for $i=1,2, \ldots, n$ and $j=1,2, \ldots, m$

$\mathfrak{d}_{i j}=\operatorname{GDIVPHFEWA} A_{\omega(t)}\left(\mathfrak{d}_{i j}\left(t_{k}\right) ; k=1,2, \ldots, r\right)$

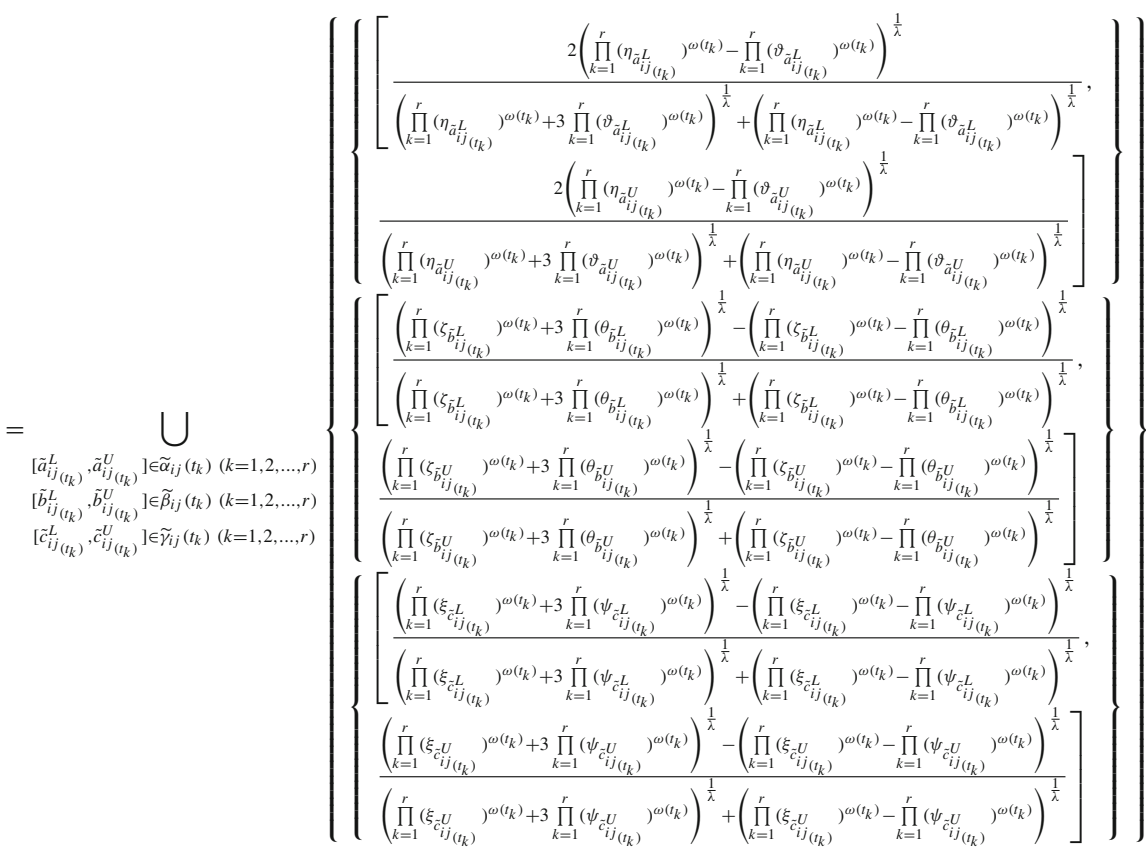

where

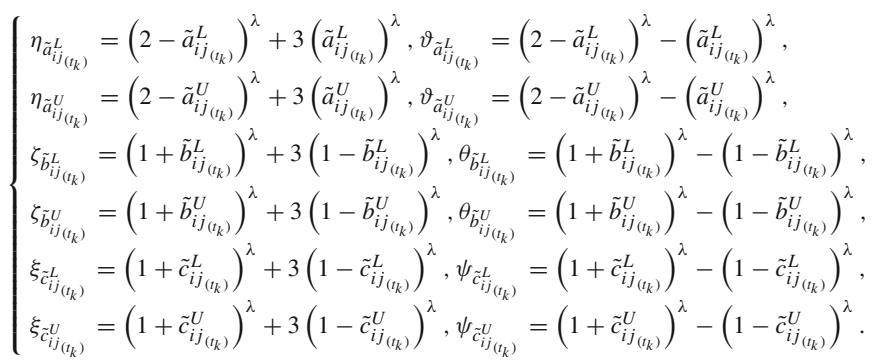


Step 3. Utilize the interval-valued picture hesitant fuzzy aggregation operators (IVPHFWA, IVPHFWG) to find the collective overall preference values $\mathfrak{d}_{i}(i=1,2, \ldots, n)$ of the alternatives $q_{i}(i=1,2, \ldots, n)$.

If we use the IVPHFWA operator then we take for $i=1,2, \ldots, n$

$$
\begin{aligned}
& \mathfrak{d}_{i}=\operatorname{IVPHFW} A_{\omega}\left(\mathfrak{d}_{i j} ; j=1,2, \ldots, m\right) \\
& =\underset{\substack{\left[\tilde{a}_{i j}^{L}, \tilde{a}_{i j}^{U}\right] \in \tilde{\alpha}_{i j}(j=1,2, \ldots, m) \\
\left[\tilde{b}_{i j}^{L}, \tilde{b}_{i j}^{U}\right] \in \tilde{\beta}_{i j}(j=1,2, \ldots, m) \\
\left[\tilde{c}_{i j}^{L}, \tilde{c}_{i j}^{U}\right] \in \tilde{\gamma}_{i j}(j=1,2, \ldots, m)}}{\bigcup}\left\{\left\{\left\{\left[\begin{array}{l}
\left.\left.1-\prod_{j=1}^{m}\left(1-\tilde{a}_{i j}^{L}\right)^{\omega_{j}}, 1-\prod_{j=1}^{m}\left(1-\tilde{a}_{i j}^{U}\right)^{\omega_{j}}\right]\right\} \\
\left.\left.\prod_{j=1}^{m}\left(\tilde{b}_{i j}^{L}\right)^{\omega_{j}}, \prod_{j=1}^{m}\left(\tilde{b}_{i j}^{U}\right)^{\omega_{j}}\right]\right\}
\end{array}\right\} .\right.\right.\right.
\end{aligned}
$$

Step 4. Same as Step 4 of Algorithm 1.

Step 5. Same as Step 5 of Algorithm 1.

To clarify the organization of the proposed algorithms, we present the the combined frame diagram of Algorithm 1 and Algorithm 2 in Fig. 2.

Example 5.1 (For Algorithm 1) Suppose that a high-level committee has been formed to investigate the impacts of COVID-19 in daily life for the four major cities $q_{1}, q_{2}, q_{3}$, and $q_{4}$ (extendable) in a country. This committee consists of COVID-19 board members appointed by the ministry of health, economy and society (government departments). The committee is directed to explore the cities based on the three most important parameters $l_{1}$-Healthcare, $l_{2}$-Economic, and $l_{3}$-Social throughout the two significant lockdown periods $t_{1}$ and $t_{2}$. The committee is analyzing the Challenges in the diagnosis, Quarantine, and remedy of suspected or confirmed cases, High burden of the functioning of the present medical system, Patients with different disorder and fitness troubles are getting neglected, Overload on doctors and different fitness care professionals, Overloading of medical shops, and Disruption of the medical supply chain under the parameter $l_{1}$-Healthcare. Slowing the production of crucial goods, Disrupt the supply chain of products, Loss in countrywide and global business, Poor cash flow in the market, and Significant slowing down in the sales boom under the parameter $l_{2}$-Economic. Service quarter isn't always being capable of offering their right service, Cancellation or postponement of large-scale sports activities and tournaments, Avoiding the countrywide and global traveling and cancellation of services, Disruption of the celebration of cultural, spiritual, and festive events, Undue strain most of the population, Social distancing with our friends and own circle of relatives members, Closure of the hotels, eating places and religious places, Closure of places for leisure which include film and play theaters, sports activities, clubs, gymnasiums, and swimming pools, Postponement of examinations under the parameter $l_{3}$-Social. Assume that $\omega=(0.2,0.3,0.5)$ be the weightage of criteria $l_{1}, l_{2}$ and $l_{3}$ and $\omega(t)=(0.6,0.4)$ be the weightage of the time periods $t_{1}$ and $t_{2}$. Suppose that experts create the idealized decision matrix table (see Table 1) which has the dynamic interval-valued picture hesitant fuzzy variables/numbers. We can find the mostly affected city in daily life by COVID-19. It is being executed through Step 1 to Step 5 of Algorithm 1 as follows.

The values provided in Table 1 consistent with the committee evaluations for the effects of COVID-19 can be interpreted as follows. From the topmost access $\{\{[0.15,0.90]\},\{[0.01$, 0.02], $[0.02,0.03]\},\{[0.04,0.05]\}\}$ of Table 1, we apprehend that $4-5 \%$ of Healthcare sector is affected, $1-2 \%$ or $2-3 \%$ of Healthcare sector is neither affected, nor benefited, and 


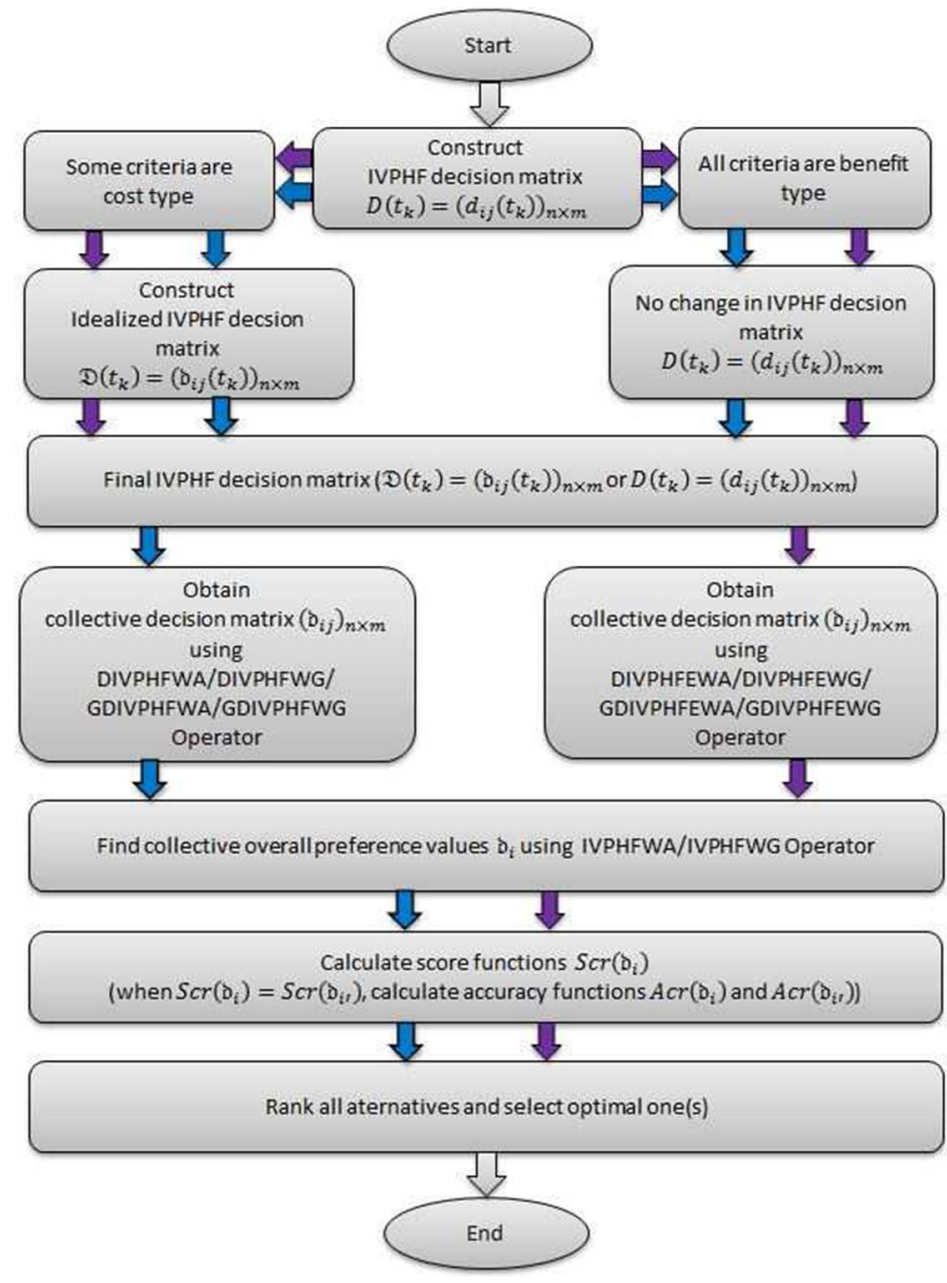

Fig. 2 Frame diagram of Algorithm 1 and Algorithm 2

$15-90 \%$ of Healthcare sector is not affected during the period $t_{1}$ for the city $q_{1}$ due to COVID19. From the second one topmost access $\{\{[0.08,0.80]\},\{[0.01,0.02]\},\{[0.14,0.16]\}\}$ of Table 1, we recognize that $14-16 \%$ of Economic sector is affected, $1-2 \%$ of Economic sector is neither affected, nor benefited, and $8-80 \%$ of Economic sector is not affected during the period $t_{1}$ for the city $q_{1}$ due to COVID-19. From the third topmost access $\{\{[0.07,0.70]\},\{[0.10,0.11]\},\{[0.01,0.02]\}\}$ of Table 1 , we understand that $1-2 \%$ of Social sector is affected, $10-11 \%$ of Social sector is neither affected nor benefited, and $7-70 \%$ of Social sector is not affected during the period $t_{1}$ for the city $q_{1}$ due to COVID-19. Similarly, we can interpret all of the values of Table 1 as well. We can find the mostly affected city due to COVID-19 through executing the Step 1 to Step 5 of Algorithm 1 as follows.

Step 1. The idealized decision matrix $\mathfrak{D}\left(t_{k}\right)=\left(\mathfrak{d}_{i j}\left(t_{k}\right)\right)_{4 \times 3}(i=1,2,3,4, j=1,2,3, k=$ $1,2)$ is constructed from Table 1 . 
Table 1 Table for idealized decision matrix

\begin{tabular}{lll}
\hline Time- & Para- & Alternative \\
moment & $q_{1}$ \\
\hline$t_{1}$ & & $\{\{[0.15,0.90]\},\{[0.01,0.02],[0.02,0.03]\},\{[0.04,0.05]\}\}$ \\
& $\{\{[0.08,0.80]\},\{[0.01,0.02]\},\{[0.14,0.16]\}\}$ \\
$l_{1}$ & $\{\{[0.07,0.70]\},\{[0.10,0.11]\},\{[0.01,0.02]\}\}$ \\
$l_{2}$ & $\{\{[0.04,0.85]\},\{[0.01,0.03]\},\{[0.01,0.03],[0.07,0.08]\}\}$ \\
& $\{\{[0.01,0.70],[0.08,0.81]\},\{[0.08,0.09]\},\{[0.01,0.03]\}\}$ \\
& $\{\{[0.07,0.65]\},\{[0.09,0.10]\},\{[0.08,0.10]\}\}$ \\
& $q_{2}$ \\
& $\{\{[0.04,0.30]\},\{[0.22,0.33],[0.28,0.40]\},\{[0.28,0.30]\}\}$ \\
& $\{\{[0.13,0.50],[0.41,0.48]\},\{[0.11,0.20]\},\{[0.18,0.30]\}\}$ \\
& $\{\{[0.18,0.30]\},\{[0.12,0.18]\},\{[0.17,0.50]\}\}$ \\
& $\{\{[0.17,0.50]\},\{[0.11,0.30]\},\{[0.08,0.20]\}\}$ \\
& $\{\{[0.27,0.38]\},\{[0.23,0.40]\},\{[0.14,0.20],[0.11,0.12]\}\}$ \\
& $\{\{[0.05,0.60]\},\{[0.04,0.20]\},\{[0.01,0.20]\}\}$ \\
& $q_{3}$ \\
& $\{\{[0.31,0.50]\},\{[0.11,0.14],[0.04,0.20]\},\{[0.21,0.28]\}\}$ \\
& $\{\{[0.28,0.40]\},\{[0.23,0.40]\},\{[0.01,0.20]\}\}$ \\
& $\{\{[0.01,0.20],[0.17,0.18]\},\{[0.20,0.30]\},\{[0.21,0.33]\}\}$ \\
& $\{\{[0.03,0.20]\},\{[0.33,0.48]\},\{[0.01,0.20]\}\}$ \\
& $\{\{[0.31,0.50]\},\{[0.13,0.18]\},\{[0.08,0.20]\}\}$ \\
& $\{\{[0.11,0.20]\},\{[0.22,0.40]\},\{[0.14,0.40],[0.11,0.33]\}\}$ \\
& $q 4$ \\
& $\{\{[0.11,0.50]\},\{[0.11,0.30]\},\{[0.05,0.20]\}\}$ \\
& $\{\{[0.17,0.30]\},\{[0.11,0.30],[0.11,0.14]\},\{[0.17,0.40]\}\}$ \\
& $\{\{[0.61,0.80]\},\{[0.08,0.10]\},\{[0.01,0.10],[0.02,0.09]\}\}$ \\
& $\{\{[0.14,0.20]\},\{[0.17,0.20]\},\{[0.41,0.60]\}\}$ \\
& $\{\{[0.26,0.50]\},\{[0.13,0.26]\},\{[0.01,0.10]\}\}$ \\
& $\{\{[0.22,0.40],[0.18,0.28]\},\{[0.13,0.30]\},\{[0.04,0.30]]\}\}$ \\
&
\end{tabular}

Step 2. The collective decision matrix $\left(\mathfrak{d}_{i j}\right)_{4 \times 3}(i=1,2,3,4, j=1,2,3)$ is constructed from Table 2 by utilizing the dynamic interval-valued picture hesitant fuzzy weighted average (DIVPHFWA) operator (Algorithm 1).

Step 3. The collective overall preference values $\mathfrak{d}_{i}(i=1,2,3,4)$ of the alternatives $q_{i}$ $(i=1,2,3,4)$ are obtained from Table 3 by utilizing the interval-valued picture hesitant fuzzy weighted average (IVPHFWA) operator.

Step 4. The score functions $\mathcal{S}\left(\mathfrak{d}_{i}\right)$ of $\mathfrak{d}_{i}(i=1,2,3,4)$ are obtained as mentioned in Table 4. Therefore, we get the ranking order of given alternatives is $q_{1} \succ q_{4} \succ q_{2} \succ q_{3}$ according to DIVPHFWA operator.

If we apply DIVPHFWG operator, we get the score values and ranking order of alternatives as mentioned in Table 4. The score values and ranking order of alternatives using the GDIVPHFWA and GDIVPHFWG operators for different values of $\lambda$ are displayed in Table 5. By considering the dynamic aggregation operators: DIVPHFWA and DIVPHFWG and the generalized dynamic aggregation operators: GDIVPHFWA 


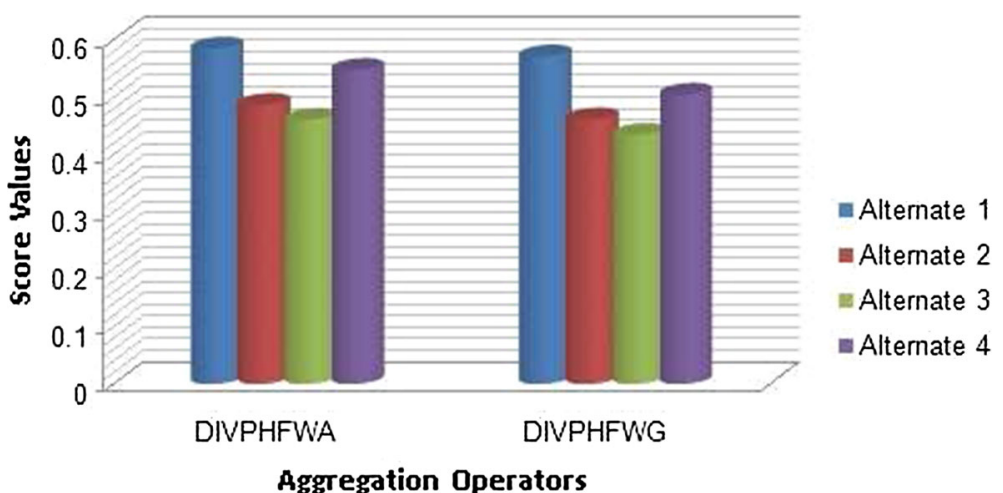

Fig. 3 Comparison chart for DIVPHFWA-DIVPHFWG operators

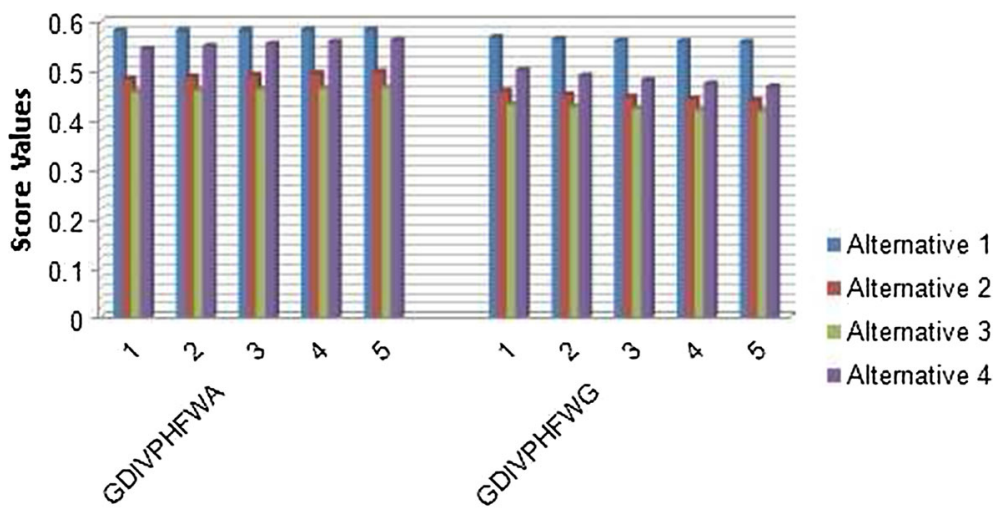

Aggregation Operators with different values of $\boldsymbol{\lambda}$

Fig. 4 Comparison chart for GDIVPHFWA-GDIVPHFWG operators with different values of $\lambda$

and GDIVPHFWG, we point out that the score values for the alternatives are different, but the ranking order of the alternatives is the same. It is clearly visible in Figs. 3 and 4 .

Example 5.2 (For Algorithm 2) To illustrate the steps of Algorithm 2, we deal with the investment opportunities selection problem. A fund manager Mr. M in a wealth management firm is assessing three potential investment opportunities $q_{1}, q_{2}, q_{3}$. The firm mandates that the fund manager has to evaluate the following three parameters $l_{1}$ and $l_{2}$, where $l_{1}$ stands for the 'risk' and $l_{2}$ stands for the 'growth'. For each parameter $l_{i}(i=1,2)$, Mr. M invites two expert groups to evaluate the $q_{j}(j=1,2,3)$ with respect to $l_{i}(i=1,2)$ in three different periods of time $t_{1}, t_{2}, t_{3}$. Assume that $\omega=(0.3,0.7)$ be the weightage of criteria $l_{1}$ and $l_{2}$ and $\omega(t)=(0.1,0.3,0.6)$ be the weightage of the time periods $t_{1}, t_{2}$, and $t_{3}$. Suppose that experts create the idealized decision matrix table (see Table 6) which has the dynamic interval-valued picture hesitant fuzzy variables/numbers. The most suitable parameter can be selected for investment through the Step 1 to Step 5 of Algorithm 2 as follows.

Step 1. The idealized decision matrix $\mathfrak{D}\left(t_{k}\right)=\left(\mathfrak{d}_{i j}\left(t_{k}\right)\right)_{3 \times 2}(i=1,2,3, j=1,2, k=$ $1,2,3)$ is constructed from Table 6. 


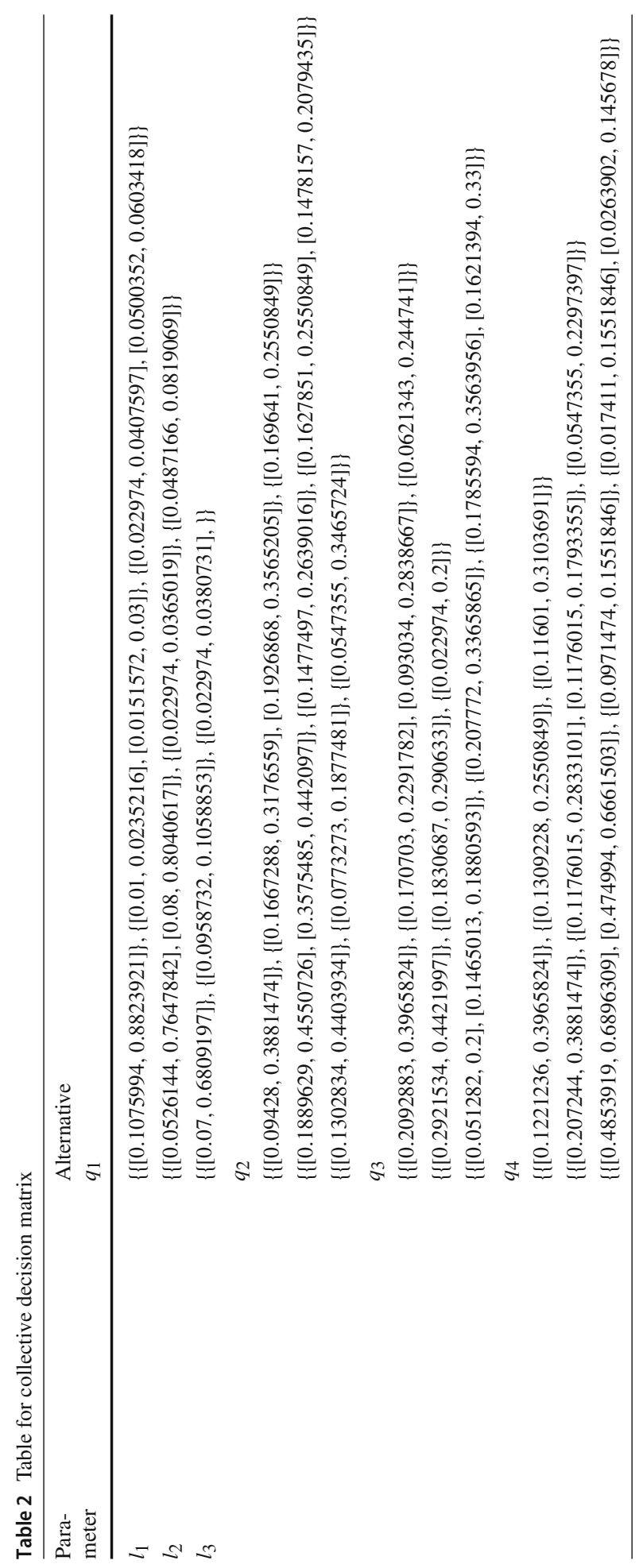




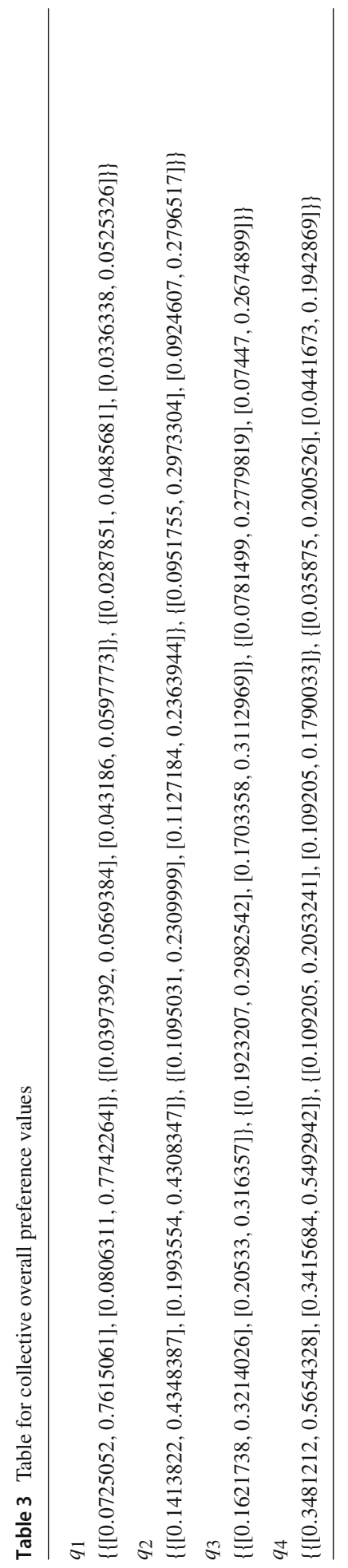


Table 4 Table of score values-ranking order for DIVPHFWA-DIVPHFWG operators

\begin{tabular}{|c|c|c|c|c|c|}
\hline Operators & Score values & & & & Ranking order \\
\hline DIVPHFWA & 0.5828568 & 0.4845111 & 0.4584353 & 0.5454265 & $q_{1} \succ q_{4} \succ q_{2} \succ q_{3}$ \\
\hline DIVPHFWG & 0.569065 & 0.4593005 & 0.432204 & 0.50235 & $q_{1} \succ q_{4} \succ q_{2} \succ q_{3}$ \\
\hline
\end{tabular}

Table 5 Ranking order of alternatives for GDIVPHFWA-GDIVPHFWG operators with different values of $\lambda$

\begin{tabular}{lllllll}
\hline Operators & $\lambda$ & Score values & & & Ranking order \\
\hline & 1 & 0.5828568 & 0.4845111 & 0.4584353 & 0.5454265 & $q_{1} \succ q_{4} \succ q_{2} \succ q_{3}$ \\
GDIVPHFWA & 2 & 0.5834922 & 0.4885244 & 0.4613326 & 0.5508532 & $q_{1} \succ q_{4} \succ q_{2} \succ q_{3}$ \\
& 3 & 0.5839548 & 0.4923313 & 0.4635947 & 0.5556214 & $q_{1} \succ q_{4} \succ q_{2} \succ q_{3}$ \\
& 5 & 0.5843024 & 0.4957437 & 0.4654296 & 0.5596157 & $q_{1} \succ q_{4} \succ q_{2} \succ q_{3}$ \\
& 1 & 0.5845858 & 0.4987449 & 0.4669893 & 0.5629243 & $q_{1} \succ q_{4} \succ q_{2} \succ q_{3}$ \\
GDIVPHFWG & 2 & 0.569065 & 0.4593005 & 0.432204 & 0.50235 & $q_{1} \succ q_{4} \succ q_{2} \succ q_{3}$ \\
& 3 & 0.5623685 & 0.4479962 & 0.424282 & 0.4816923 & $q_{1} \succ q_{4} \succ q_{2} \succ q_{3}$ \\
& 4 & 0.5606428 & 0.4439221 & 0.4214459 & 0.4744388 & $q_{1} \succ q_{4} \succ q_{2} \succ q_{3}$ \\
& 5 & 0.5594333 & 0.4406658 & 0.4191625 & 0.4688665 & $q_{1} \succ q_{4} \succ q_{2} \succ q_{3}$ \\
\hline
\end{tabular}

Table 6 Table for idealized decision matrix

\begin{tabular}{|c|c|c|}
\hline Time-moment & Parameter & Alternative $q_{1}$ \\
\hline \multirow[t]{2}{*}{$t_{1}$} & $l_{1}$ & $\{\{[0.05,0.10]\},\{[0.10,0.30]\},\{[0.12,0.20],[0.10,0.30]\}\}$ \\
\hline & $l_{2}$ & $\{\{[0.14,0.30]\},\{[0.21,0.40]\},\{[0.15,0.30]\}\}$ \\
\hline \multirow[t]{2}{*}{$t_{2}$} & $l_{1}$ & $\{\{[0.14,0.40]\},\{[0.03,0.10]\},\{[0.02,0.03],[0.01,0.10]\}\}$ \\
\hline & $l_{2}$ & $\{\{[0.21,0.30],[0.18,0.21]\},\{[0.32,0.40]\},\{[0.01,0.10]\}\}$ \\
\hline \multirow[t]{16}{*}{$t_{3}$} & $l_{1}$ & $\{\{[0.22,0.40]\},\{[0.03,0.20]\},\{[0.01,0.04]\}\}$ \\
\hline & $l_{2}$ & $\{\{[0.21,0.50]\},\{[0.05,0.10]]\},\{[0.03,0.20]\}\}$ \\
\hline & & $q_{2}$ \\
\hline & & $\{\{[0.14,0.30]\},\{[0.02,0.13],[0.08,0.20]\},\{[0.18,0.20]\}\}$ \\
\hline & & $\{\{[0.02,0.10]\},\{[0.01,0.10]\},\{[0.19,0.20]\}\}$ \\
\hline & & $\{\{[0.16,0.50]\},\{[0.01,0.20]\},\{[0.05,0.10]\}\}$ \\
\hline & & $\{\{[0.17,0.18],[0.16,0.30]\},\{[0.04,0.20]\},\{[0.15,0.30]\}\}$ \\
\hline & & $\{\{[0.13,0.30]\},\{[0.13,0.28]\},\{[0.01,0.10]\}\}$ \\
\hline & & $\{\{[0.21,0.40]\},\{[0.13,0.28]\},\{[0.08,0.30]\}\}$ \\
\hline & & $q_{3}$ \\
\hline & & $\{\{[0.01,0.20]\},\{[0.04,0.30]\},\{[0.11,0.18],[0.04,0.20]\}\}$ \\
\hline & & $\{\{[0.18,0.20]\},\{[0.03,0.10]\},\{[0.02,0.21]\}\}$ \\
\hline & & $\{\{[0.03,0.10]\},\{[0.13,0.18],[0.21,0.40]\},\{[0.01,0.10]\}\}$ \\
\hline & & $\{\{[0.21,0.40]\},\{[0.13,0.18],[0.12,0.27]\},\{[0.08,0.10]\}\}$ \\
\hline & & $\{\{[0.03,0.10]\},\{[0.13,0.28]\},\{[0.01,0.30]\}\}$ \\
\hline & & $\{\{[0.01,0.20]\},\{[0.03,0.28]\},\{[0.08,0.10]\}\}$ \\
\hline
\end{tabular}




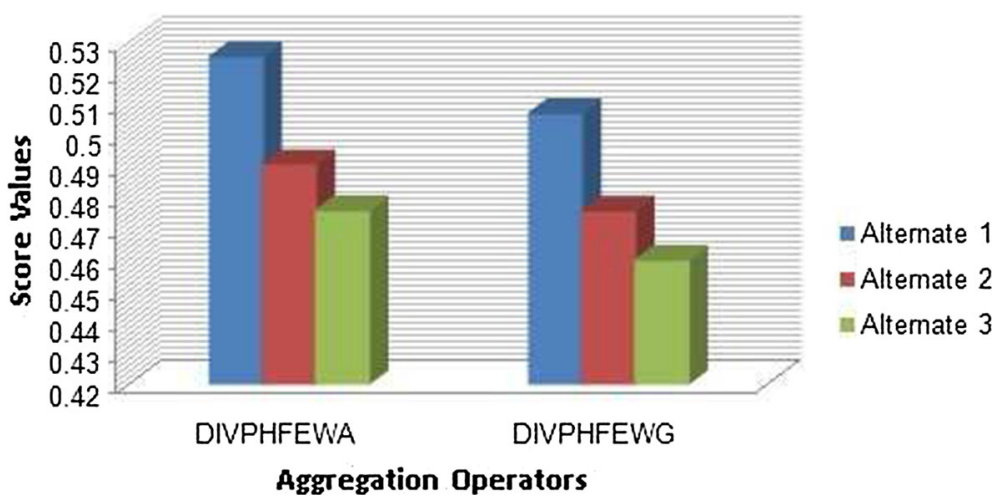

Fig. 5 Comparison chart for DIVPHFEWA-DIVPHFEWG operators

Step 2. The collective decision matrix $\left(\mathfrak{d}_{i j}\right)_{3 \times 2}(i=1,2,3, j=1,2)$ is constructed from Table 7 by utilizing the interval-valued picture hesitant fuzzy Einstein weighted average (IVPHFEWA) operator (Algorithm 2).

Step 3. The collective overall preference values $\mathfrak{d}_{i}(i=1,2,3)$ of the alternatives $q_{i}(i=$ $1,2,3)$ are obtained from Table 8 by utilizing the interval-valued picture hesitant fuzzy weighted average (IVPHFWA) operator.

Step 4. The score functions $\mathcal{S}\left(\mathfrak{d}_{i}\right)$ of $\mathfrak{d}_{i}(i=1,2,3)$ are obtained as mentioned in Table 9. Therefore, we get the ranking order of given alternatives is $q_{1} \succ q_{2} \succ q_{3}$ according to DIVPHFEWA operator.

If we apply DIVPHFEWG operator, we get the score values and ranking order of alternatives as mentioned in Table 9. The score values and ranking order of alternatives using the GDIVPHFEWA and GDIVPHFEWG operators for different values of $\lambda$ are displayed in Table 10. By considering the dynamic aggregation operators based on Einstein operations: DIVPHFEWA and DIVPHFEWG and the generalized dynamic aggregation operators based on Einstein operations: DIVPHFEWA and DIVPHFEWG, we point out that the score values for the alternatives are different, but the ranking order of the alternatives is the same. It is visible through Figs. 5 and 6.

\section{Limitations of the proposed approaches:}

To reveal the limitations of the proposed approaches, we analyze Algorithm 1 and Algorithm 2 critically and give their drawbacks as follows.

In the above examples, possible dependencies between criteria (parameters) are neglected. It is practically not always possible to assume in the MPDM that each parameter is independent of other parameters. Any parameter in the MPDM could be related to, or dependent on other parameters. In the proposed MPDM approaches, evaluating the dependencies among parameters should contribute to the objectivity of decisions. The considering dependency in the interval-valued picture hesitant fuzzy MPDM may improve the quality of decision-making process. 


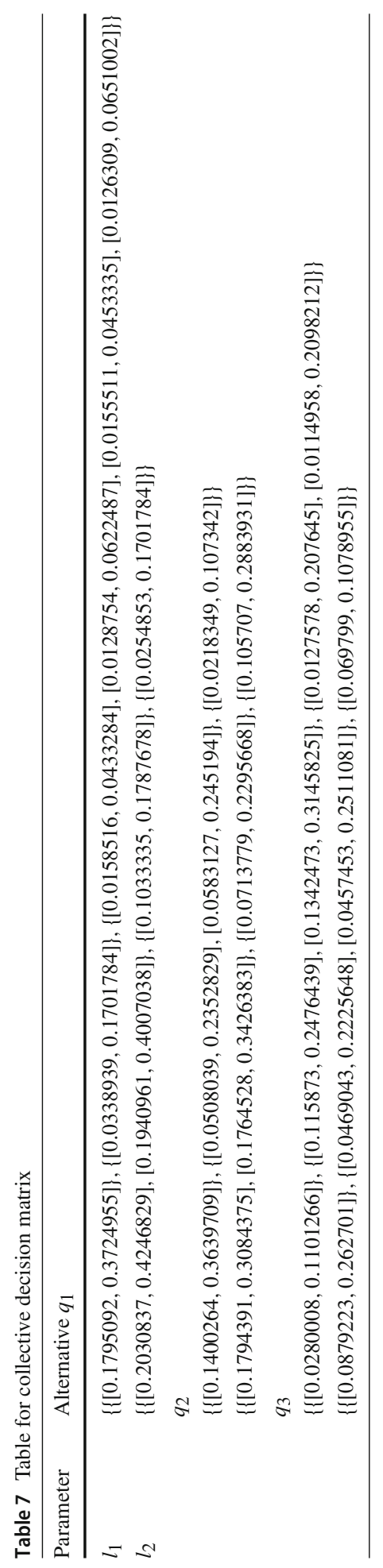




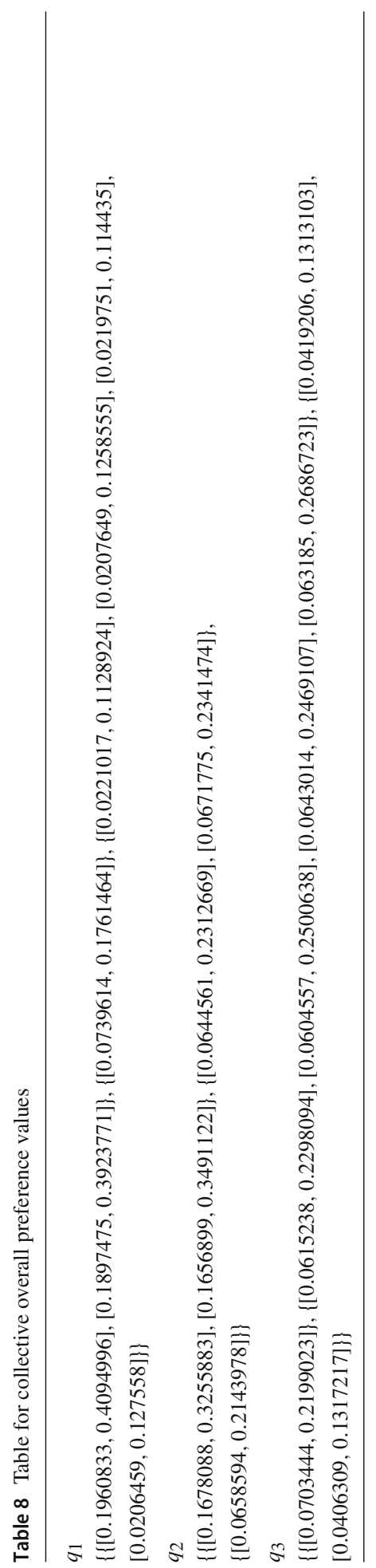


Table 9 Table of score values-ranking order for DIVPHFEWA-DIVPHFEWG operators

\begin{tabular}{|c|c|c|c|c|}
\hline Operators & Score values & & & Ranking order \\
\hline DIVPHFEWA & 0.5252736 & 0.4906648 & 0.4757781 & $q_{1} \succ q_{2} \succ q_{3}$ \\
\hline DIVPHFEWG & 0.5070679 & 0.4755731 & 0.4598301 & $q_{1} \succ q_{2} \succ q_{3}$ \\
\hline
\end{tabular}

Table 10 Ranking order alternatives for GDIVPHFEWA-GDIVPHFEWG operators with different values of $\lambda$

\begin{tabular}{llllll}
\hline Operators & $\lambda$ & Score functions & & Ranking Order \\
\hline & 1 & 0.5252736 & 0.4906648 & 0.4757781 & $q_{1} \succ q_{2} \succ q_{3}$ \\
GDIVPHFEWA & 2 & 0.5279883 & 0.4937191 & 0.4819029 & $q_{1} \succ q_{2} \succ q_{3}$ \\
& 3 & 0.5307157 & 0.4960927 & 0.4861892 & $q_{1} \succ q_{2} \succ q_{3}$ \\
& 4 & 0.5331643 & 0.4980722 & 0.4895613 & $q_{1} \succ q_{2} \succ q_{3}$ \\
GDIVPHFEWG & 5 & 0.5353295 & 0.4998603 & 0.4924096 & $q_{1} \succ q_{2} \succ q_{3}$ \\
& 1 & 0.5070679 & 0.4755731 & 0.4598301 & $q_{1} \succ q_{2} \succ q_{3}$ \\
& 2 & 0.4918949 & 0.4703409 & 0.4553754 & $q_{1} \succ q_{2} \succ q_{3}$ \\
& 4 & 0.4795927 & 0.4658424 & 0.4516602 & $q_{1} \succ q_{2} \succ q_{3}$ \\
& 5 & 0.4703139 & 0.4618056 & 0.4486142 & $q_{1} \succ q_{2} \succ q_{3}$ \\
& 0.4630513 & 0.4581448 & 0.4460911 & $q_{1} \succ q_{2} \succ q_{3}$ \\
\hline
\end{tabular}

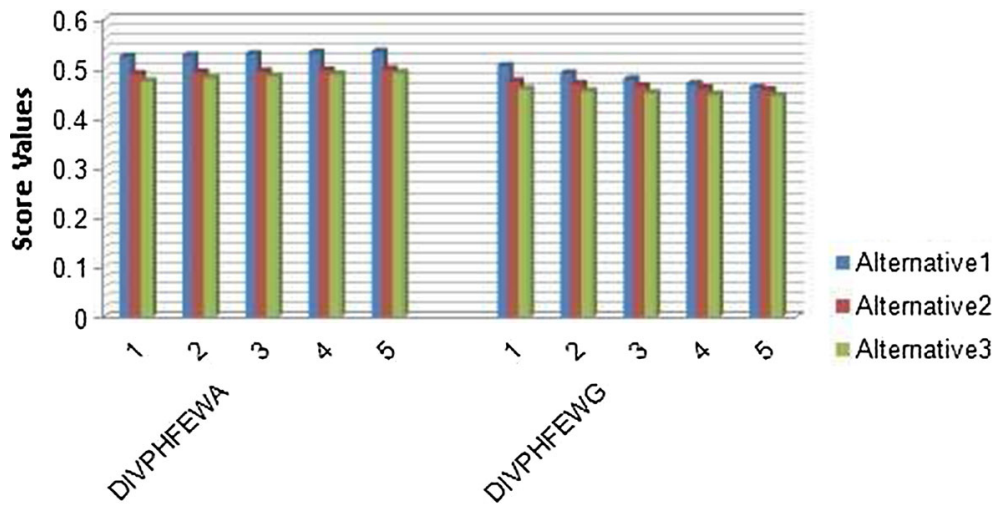

\section{Aggregation Operators with different values of $\lambda$}

Fig. 6 Comparison chart for GDIVPHFEWA-GDIVPHFEWG operators with different values of $\lambda$

\section{Comparative study}

To show the feasibleness of the proposed MPDM strategies, the rankings of our proposed operators DIVPHFWA, DIVPHFEWA, DIVPHFWG and DIVPHFEWG are compared with the rankings obtained by the existing MPDM methods as presented in Table 11. The hesitant fuzzy set could be a special case of IVPHFS. For instance, the component $\left(Y_{1}, G_{1}\right)=(0.2,0.4,0.7)$ in Table 1 of Xia and $\mathrm{Xu}$ (2011) will be viewed as an IVPHFE as $\{\{(0.2,0.2),(0.4,0.4),(0.7,0.7)\},\{(0,0)\},\{(0,0)\}\}$. A dual hesitant fuzzy set is a special case of IVPHFS due to the fact the element $\left(A_{1}, C_{1}\right)=$ 


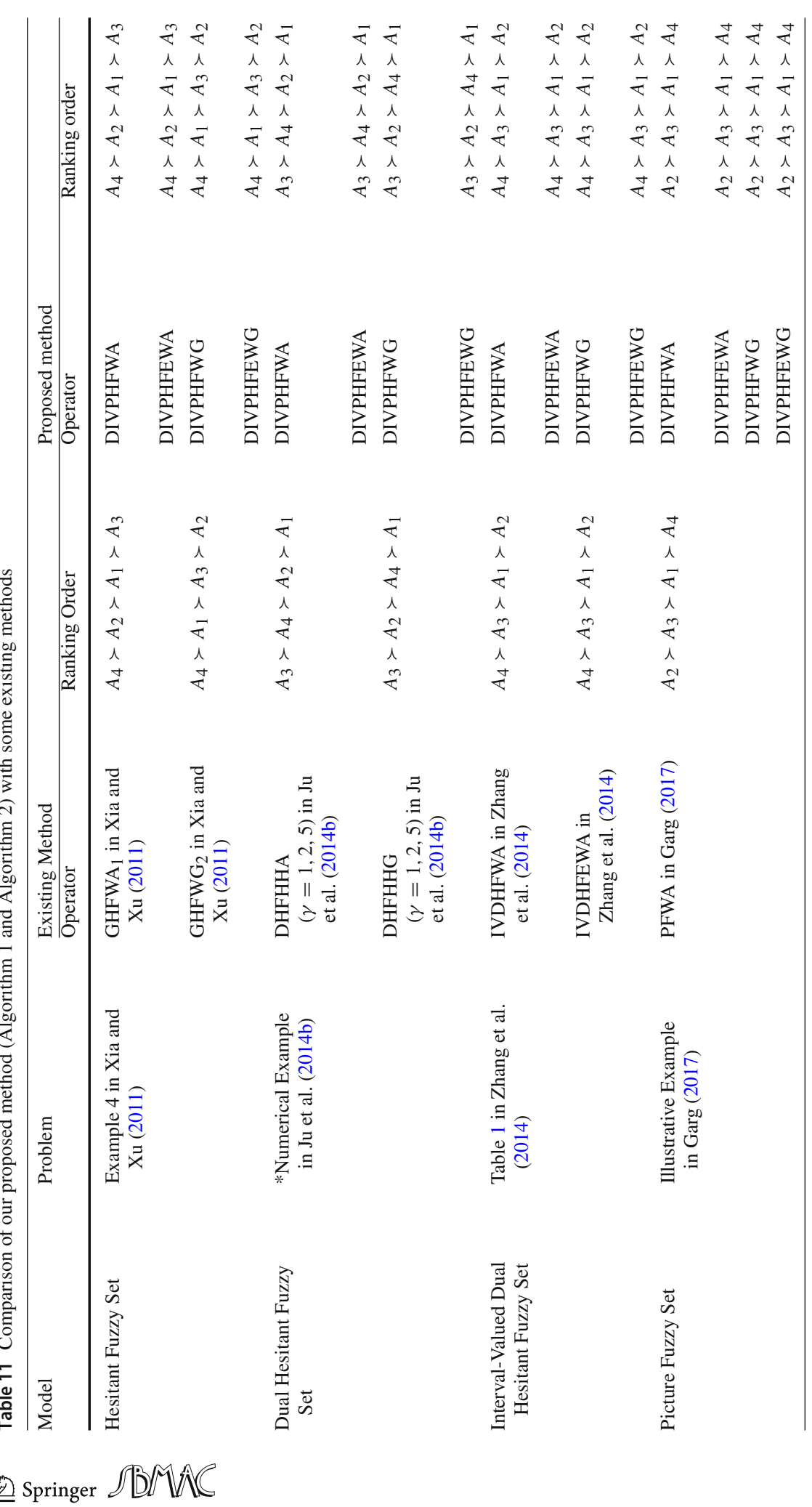




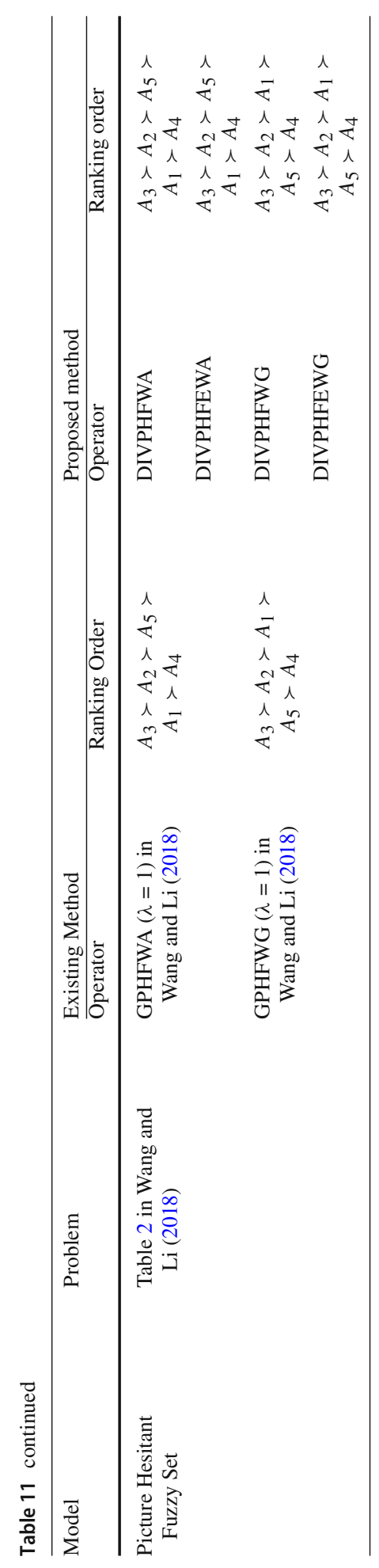


$\{\{0.3,0.4\},\{0.6\}\}$ in Table 1 of $\mathrm{Ju}$ et al. (2014b) can be converted into IVPHFE as $\{\{(0.3,0.3),(0.4,0.4)\},\{(0,0)\},\{(0.6,0.6)\}\}$. The interval-valued dual hesitant fuzzy element $\left(A_{1}, C_{1}\right)=\{\{[0.3,0.4],[0.4,0.5],[0.5,0.6]\},\{[0.2,0.3],[0.2,0.4]\}\}$ in Table 1 of Zhang et al. (2014) is transformed to IVPHFE as $\{\{[0.3,0.4],[0.4,0.5],[0.5,0.6]\},\{[0,0]\}$, $\{[0.2,0.3],[0.2,0.4]\}\}$. The picture fuzzy element $\left(A_{1}, G_{1}\right)=(0.2,0.1,0.6$,$) in illustrative$ Example of Garg (2017) is modified to IVPHFE as $\{\{(0.2,0.2)\},\{(0.1,0.1)\},\{(0.6,0.6)\}\}$. Moreover, Picture hesitant fuzzy element $\left(A_{1}, C_{1}\right)=\{\{0.4,0.5,0.7\},\{0.05\},\{0.1,0.2\}\}$ in Table 2 of Wang and Li (2018) also can be modified to IVPHFE as $\{\{(0.4,0.4),(0.5,0.5)$, $(0.7,0.7)\},\{(0.05,0.05)\},\{(0.1,0.1),(0.2,0.2)\}\}$. It leads to comparing our proposed model IVPHFS with the existing models HFS, DHFS, IVDHFS, PFS, and PHFS. Our proposed operators DIVPHFWA, DIVPHFEWA, DIVPHFWG and DIVPHFEWG provide the identical ranking in evaluating with the prevailing operators $\mathrm{GHFWA}_{1}, \mathrm{GHFWA}_{2} \mathrm{Xia}$ and $\mathrm{Xu}$ (2011), DHFHHA, DHFHHG ( $\gamma=1,2,5) \mathrm{Ju}$ et al. (2014b), IVDHFWA, IVDHFEWA Zhang et al. (2014), PFWA Garg (2017), GPHFWA, GPHFWA $(\lambda=1)$ Wang and Li (2018) of the models HFS, DHFS, IVDHFS, PFS, and PHFS, respectively. It is proven in Table 11.

\section{Conclusion}

In this paper, we studied and described the notions and operations of IVPHFSs and IVPHFEs. Some basic properties of the IVPHFEs were also introduced and investigated. We discussed the MPDM problems under interval-valued picture hesitant fuzzy environment. Then, motivated by the idea of traditional dynamic aggregation operators and Einstein operations, some dynamic interval-valued picture hesitant aggregation operators: DIVPHFWA operator, DIVPHFWG operator, DIVPHFEWA operator, DIVPHFEWG operator, and their generalized versions were proposed to aggregate the interval-valued picture hesitant fuzzy information collected at different periods. In addition, we proposed these dynamic aggregation operators to develop new approaches to deal with the interval-valued picture hesitant fuzzy multi-period decision-making problems. The practical examples and a comparison study were presented to verify the practicality and effectiveness of newly proposed approaches.

The IVPHFS introduced in this paper is quite an extensive version of many (extended) fuzzy sets, and hence the proposed aggregation operators for IVPHFVs are more inclusive than those of many fuzzy variables. In addition, the novelty of the interval-valued picture hesitant fuzzy aggregation operators proposed in this paper is that they can realize the synthesis of the interval-valued picture hesitant fuzzy information collected at different periods, so they develop and enrich the theories of dynamic aggregation operators for hesitant fuzzy elements, picture fuzzy elements, picture hesitant fuzzy elements.

In the future, we will extend the theory of aggregation operators for IVPHFVs and investigate information measures to deal with the decision-making problems in medical diagnosis and pattern recognition under interval-valued picture hesitant fuzzy environment.

\section{Declarations}

Conflict of interest The authors declare that there is no conflict of interest regarding the publication of this paper.

Funding: This research received no external funding. 


\section{References}

Akram M, Ishfaq N, Sayed S, Smarandache F (2018) Decision-making approach based on neutrosophic rough information. Algorithms 11(5):59

Atanassov KT (1986) Intuitionistic fuzzy sets. Fuzzy Sets Syst 20:87-96

Atanassov KT (1989) More on intuitionistic fuzzy sets. Fuzzy Sets Syst 33:37-46

Atanassov K, Gargov G (1989) Interval-valued intuitionistic fuzzy sets. Fuzzy Sets Syst 31:343-349

Beg I, Rashid T (2014) Group decision making using intuitionistic hesitant fuzzy sets. Int J Fuzzy Logic Intell Syst 14(3):181-187

Cuong BC (2014) Picture fuzzy sets. J Comput Sci Cybern 30(4):409-420

Chen N, Xu Z, Xia M (2013) Interval-valued hesitant preference relations and their applications to group decision making. Knowl Based Syst 37:528-540

Deli I, Karaaslan F (2020) Generalized trapezoidal hesitant fuzzy numbers and their applications to multi criteria decision-making problems. Soft Comput. https://doi.org/10.1007/s00500-020-05201-2 (in press)

Garg H (2017) Some picture fuzzy aggregation operators and their applications to multicriteria decisionmaking. Arab J Sci Eng 42:5275-5290

Jin F, Ni Z, Chen H (2016) Interval-valued hesitant fuzzy Einstein prioritized aggregation operators and their applications to multi-attribute group decision making. Soft Comput 20:1863-1878

Ju Y, Liu X, Yang S (2014) Interval-valued dual hesitant fuzzy aggregation operators and their applications to multiple attribute decision making. J Intell Fuzzy Syst 27:1203-1218

Ju Y, Zhang W, Yang S (2014) Some dual hesitant fuzzy Hamacher aggregation operators and their applications to multiple attribute decision making. J Intell Fuzzy Syst 27(5):2481-2495

Kamaci H (2020) Neutrosophic cubic Hamacher aggregation operators and their applications in decision making. Neutrosophic Sets Syst 33:234-255

Kamac1 H (2019) Interval-valued fuzzy parameterized intuitionistic fuzzy soft sets and their applications. Cumhuriyet Sci J 40(2):317-331

Karaaslan F, Karataş S (2016) OR and AND-products of IFP-intuitionistic fuzzy soft sets and their applications in decision making. J Intell Fuzzy Syst 31(3):1427-1434

Khalil AM, Li S-G, Garg H, Li H, Ma S (2019) New operations on interval-valued picture fuzzy set, intervalvalued picture fuzzy soft set and their applications. IEEE Access 7:51236-51253

Khan A, Zhu Y (2020) New algorithms for parameter reduction of intuitionistic fuzzy soft sets. Comput Appl Math 39:232

Khan MJ, Kumam P, Liu P, Kumam W (2019) A novel approach to generalized intuitionistic fuzzy soft sets and its application in decision support system. Mathematics 7(8):742

Khan S, Abdullah S, Ashraf S (2019) Picture fuzzy aggregation information based on Einstein operations and their application in decision making. Math Sci 13:213-229

Klement EP, Mesiar R, Pap E (2004) Triangular norms. Position paper I: basic analytical and algebraic properties. Fuzzy Sets Syst 143(1):5-26

Lin YH, Lee PC, Ting H (2008) Dynamic multi-attribute decision making model with grey number evaluations. Expert Syst Appl 35:1638-1644

Naeem K, Riaz M, Peng X, Afzal D (2019) Pythagorean fuzzy soft MCGDM methods based on TOPSIS, VIKOR and aggregation operators. J Intell Fuzzy Syst 37(5):6937-6957

Peng D, Wang H (2014) Dynamic hesitant fuzzy aggregation operators in multi-period decision making. Kybernetes 43(5):715-736

Petchimuthu S, Garg H, Kamacı H, Atagün AO (2020) The mean operators and generalized products of fuzzy soft matrices and their applications in MCGDM. Comput Appl Math 39(2):68

Petchimuthu S, Kamaci H (2020) The adjustable approaches to multi-criteria group decision making based on inverse fuzzy soft matrices. Sci Iran. https://doi.org/10.24200/sci.2020.54294.3686 (in press)

Riaz M, Hashmi MR (2020) m-polar neutrosophic soft mapping with application to multiple personality disorder and its associated mental disorders. Artificial Intelligence Review (in press). https://doi.org/10. 1007/s10462-020-09912-8

Riaz M, Naeem K, Afzal D (2020) A similarity measure under Pythagorean fuzzy soft environment with applications. Comput Appl Math 39:269

Riaz M, Tehrim ST (2019) Cubic bipolar fuzzy ordered weighted geometric aggregation operators and their application using internal and external cubic bipolar fuzzy data. Comput Appl Math 38(2):87

Riaz M, Tehrim ST (2020) On bipolar fuzzy soft topology with decision-making. Soft Comput 24:1825918272

Sambuc R (1975) Fonctions $\Phi$-floues. Application a 1'aide au diagnostic en pathologie thyroidienne. Ph.D. Thesis, Univ. Marseille, France 
Smarandache F (1998) Neutrosophy: neutrosophic probability, set, and logic: analytic synthesis \& synthetic analysis. American Research Press, Rehoboth

Tehrim ST, Riaz M (2019) A novel extension of TOPSIS to MCGDM with bipolar neutrosophic soft topology. J Intell Fuzzy Syst 37(4):5531-5549

Torra V (2010) Hesitant fuzzy sets. Int J Intell Syst 25:529-539

Wang H, Zhao X, Wei G (2014) Dual hesitant fuzzy aggregation operators in multiple attribute decision making. J Intell Fuzzy Syst 26(5):2281-2290

Wang L, Garg H, Li N (2020) Pythagorean fuzzy interactive Hamacher power aggregation operators for assessment of express service quality with entropy weight. Soft Comput. https://doi.org/10.1007/s00500020-05193-z (in press)

Wang L, Li N (2020) Pythagorean fuzzy interaction power Bonferroni mean aggregation operators in multiple attribute decision making. Int J Intell Syst 35:150-183

Wang R, Li Y (2018) Picture hesitant fuzzy set and its application to multiple criteria decision-making. Symmetry 10:295. https://doi.org/10.3390/sym10070295

Wei G (2012) Hesitant fuzzy prioritized operators and their application to multiple attribute decision making. Knowl Based Syst 31:176-182

Wei G (2017) Picture fuzzy aggregation operators and their application to multiple attribute decision making. J Intell Fuzzy Syst 33(2):713-724

Wei G (2018) Picture fuzzy Hamacher aggregation operators and their application to multiple attribute decision making. Fundamenta Informaticae 157(3):271-320

Wei G, Zhao X, Lin R (2013) Some hesitant interval-valued fuzzy aggregation operators and their applications to multiple attribute decision making. Knowl Based Syst 46:43-53

Xia M, Xu Z (2011) Hesitant fuzzy information aggregation in decision making. Int J Approx Reason 52(3):395-407

Zadeh LA (1965) Fuzzy sets. Inf Control 8(3):338-353

Zang Y, Sun W, Han S (2017) Grey relational projection method for multiple attribute decision making with interval-valued dual hesitant fuzzy information. J Intell Fuzzy Syst 33(2):1053-1066

Zhang W, Li X, Ju Y (2014) Some aggregation operators based on Einstein operations under interval-valued dual hesitant fuzzy setting and application. Mathe Prob Eng 2014:1-15

Zhang Z (2013) Hesitant fuzzy power aggregation operators and their application to multiple attribute group decision making. Inf Sci 234:150-181

Zhang Z (2013) Interval-valued intuitionistic hesitant fuzzy aggregation operators and their application in group decision-making. J Appl Math 2013:33

Zhu B, Xu ZS, Xia MM (2012) Dual hesitant fuzzy sets. J Appl Math 2012:13

Zhu B, Xu ZS, Xia MM (2012) Hesitant fuzzy geometric Bonferroni means. Inf Sci 205:72-85

Publisher's Note Springer Nature remains neutral with regard to jurisdictional claims in published maps and institutional affiliations. 